\title{
Parametric validations of analytical lifetime estimates for radiation belt electron diffusion by whistler waves
}

\author{
A. V. Artemyev ${ }^{1,2}$, D. Mourenas ${ }^{3}$, O. V. Agapitov $^{1,4,5}$, and V. V. Krasnoselskikh ${ }^{1}$ \\ ${ }^{1}$ UMR7328, LPC2E/CNRS - University of Orleans, Orleans, France \\ ${ }^{2}$ Space Research Institute, RAS, Moscow, Russia \\ ${ }^{3}$ CEA, DAM, DIF, 91297, Arpajon, France \\ ${ }^{4}$ Le Studium, Institute for Advanced Studies, Orleans, France \\ ${ }^{5}$ National Taras Shevchenko University of Kiev, Kiev, Ukraine \\ Correspondence to: A. V. Artemyev (ante0226@gmail.com)
}

Received: 23 January 2013 - Revised: 11 March 2013 - Accepted: 11 March 2013 - Published: 5 April 2013

\begin{abstract}
The lifetimes of electrons trapped in Earth's radiation belts can be calculated from quasi-linear pitch-angle diffusion by whistler-mode waves, provided that their frequency spectrum is broad enough and/or their average amplitude is not too large. Extensive comparisons between improved analytical lifetime estimates and full numerical calculations have been performed in a broad parameter range representative of a large part of the magnetosphere from $L \sim 2$ to 6 . The effects of observed very oblique whistler waves are taken into account in both numerical and analytical calculations. Analytical lifetimes (and pitch-angle diffusion coefficients) are found to be in good agreement with full numerical calculations based on CRRES and Cluster hiss and lightning-generated wave measurements inside the plasmasphere and Cluster lower-band chorus waves measurements in the outer belt for electron energies ranging from $100 \mathrm{keV}$ to $5 \mathrm{MeV}$. Comparisons with lifetimes recently obtained from electron flux measurements on SAMPEX, SCATHA, SAC-C and DEMETER also show reasonable agreement.
\end{abstract}

Keywords. Magnetospheric physics (Energetic particles, precipitating; Energetic particles, trapped) - Space plasma physics (Wave-particle interactions)

\section{Introduction}

Quantifying the lifetimes of electrons trapped in Earth's radiation belts has become an increasingly important goal as scientists strive to predict high-energy particle fluxes, which may damage spaceborne high-technology systems (Iucci et al., 2005; Choi et al., 2011). However, observed flux variations related to the geomagnetic activity can reach orders of magnitudes over timescales of minutes to days (e.g., see Horne et al., 2005; Tu et al., 2010). The dynamics of the radiation belts is defined by a number of competing processes such as convection, radial diffusion driven by ultra-low frequency (ULF) waves, pitch-angle, energy, and mixed diffusion by whistler, magnetosonic and electromagnetic ion cyclotron (EMIC) waves as well as trapping by the most intense waves (Thorne, 2010). While state-of-the-art numerical models of the belts attempt to treat all these phenomena simultaneously (Barker et al., 2005; Varotsou et al., 2008; Xiao et al., 2009; Fok et al., 2011; Subbotin et al., 2011; Reeves et al., 2012) as required for space weather forecasting, one difficulty consists in evaluating electron lifetimes by computing quasi-linear diffusion coefficients (Lyons et al., 1971, 1972; Lyons, 1974; Albert, 2005; Glauert and Horne, 2005; Summers, 2005; Summers et al., 2007; Albert, 2007). Multidimensional simulations of radiation belt dynamics require computing bounce-averaged diffusion rate matrices for a very wide variety of evolving geophysical conditions (i.e., for many different values of $L$, MLT (magnetic local time), $\mathrm{Kp}$, as well as plasma density, wave parameters, and electron energy). The diffusion rate matrix can be efficiently and 
accurately computed in full diffusion codes such as the one at UCLA, by performing dedicated MPI runs (Ni et al., 2008; Shprits and Ni, 2009). Nevertheless, it still needs hours (at least) to be calculated (Gu et al., 2012). Therefore, analytical lifetime expressions can be useful substitutes to allow more rapid global belt forecasting (Li et al., 2007; Kondrashov et al., 2011; Reeves et al., 2012), parametric and sensitivity studies (Tu et al., 2009), as well as to facilitate attempts at determining the relative weight of the different source, transport, and loss processes in the Van Allen belt dynamics (Varotsou et al., 2008; Kim et al., 2011). The analytical lifetimes can also be compared with lifetimes obtained by different data-assimilation methods (Tu et al., 2010; Kondrashov et al., 2011), which do not provide by themselves any information on the relative contribution of the various sources and losses. Moreover, the rate of electron precipitation is also important to model accurately the magnetosphere-ionosphere coupling (Gkioulidou et al., 2012). Among the different types of wave-particle interactions that should be considered, the quasi-linear (stochastic) pitch-angle diffusion of electrons over their bounce motion by ensembles of uncorrelated frequency-varying whistler waves of moderate averaged amplitude (Tao et al., 2011) is known to play a prominent role as one of the major loss mechanisms in the radiation belts. Whistler mode waves scatter trapped electrons into the loss cone, where they are quickly removed by collisions with atmospheric particles (Kennel and Petschek, 1966; Trakhtengerts, 1966). The corresponding analytical lifetime expressions may be obtained either by fitting numerical calculations (as it was done for moderately oblique chorus waves by Shprits et al., 2007; Gu et al., 2012) or from complicated, approximate analytical developments (as it was done for parallel to very oblique hiss and chorus waves by Mourenas and Ripoll, 2012; Mourenas et al., 2012b,a). While numerical fits have their own advantages (a known accuracy over a restricted parameter domain), they also suffer from the limited parameter range that is considered and the associated assumptions on the wave and plasma conditions. On the other hand, analytically derived formulas may be less accurate over some domains while retaining physically (and quantitatively) correct variations over a much broader parameter range. It is precisely the main goal of the present paper to investigate extensively the accuracy of the analytical lifetime model introduced in earlier articles (Mourenas and Ripoll, 2012; Mourenas et al., 2012b), by means of numerous comparisons with full numerical calculations over a wide parameter domain representative of the whole inner magnetosphere. Determining these quasi-linear lifetimes is indeed a prerequisite for a comprehensive radiation belt modeling in the magnetosphere of Earth as well as other planets (Shprits et al., 2012).

In this paper we obtain diffusion coefficients (and corresponding estimates of lifetime) valid in a wide range of $L$ shells $(L>2)$. Thus, a broad range of the various wave parameters (frequency, wave-normal angles, etc.) and plasma density is considered. To this aim, the full Appleton-Hartree
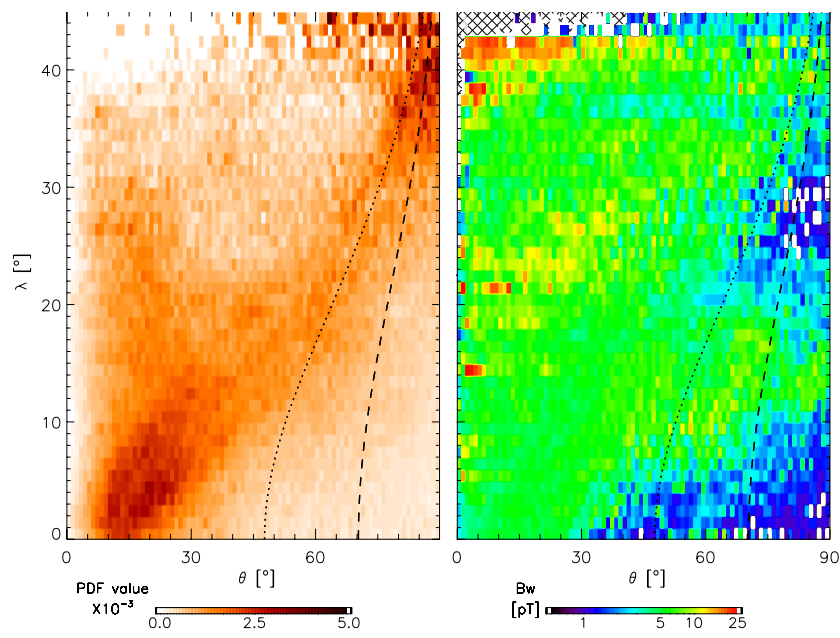

Fig. 1. Distribution of lower-band chorus occurrences in the $\theta-$ $\lambda$ space (left panel) and wave amplitude distribution (right panel) shown for the day sector and $4<L<5$ in the moderate to medium geomagnetic activity range $\mathrm{Kp}<3$ (shaded regions indicate insufficient statistics). Black curves show Gendrin and resonance cone angles for $3 \mathrm{kHz}$ at $L=4.5$.

whistler-mode dispersion relation is now used in our full numerical calculations, allowing us to extend the domain of validity of the numerical calculations. Moreover, the new analytical lifetime model presented here includes several significative improvements over the previous model (Mourenas and Ripoll, 2012; Mourenas et al., 2012a,b), which were all needed to account for (1) multiple-Gaussians wave spectra (especially inside the plasmasphere), (2) high-latitude Landau resonance at low energy and low $L<3$, and (3) highenergy, high-density cyclotron resonance at large $L>4$ in the presence of an upper latitude cutoff in the wave intensity distribution.

In a first section, the typical frequency spectra, wavenormal angle distribution and wave-power distribution with latitude of whistler waves observed onboard Cluster are presented. They are later used in full numerical calculations. The second section gives a rapid description of the complete, refined analytical lifetime model. The next section is devoted to the numerical code scheme used for calculating electron lifetimes in the presence of oblique waves. The final section will focus on an exhaustive comparison between the analytical lifetime estimates and the full numerical solutions. It will demonstrate that the analytical lifetime estimates remain generally within a factor of 2 of the actual values in a very wide parameter range.

\section{Low frequency whistler waves in the magnetosphere: a quick overview of recent statistical results}

Chorus waves typically appear in two distinct frequency bands. Lower band chorus waves appear in the range 
$0.1-0.5 \Omega_{\mathrm{c} 0}$ and upper band chorus between $0.5 \Omega_{\mathrm{c} 0}$ and $\Omega_{\mathrm{c} 0}$ where $\Omega_{\mathrm{c}}$ is the electron cyclotron frequency and $\Omega_{\mathrm{c} 0}$ is $\Omega_{\mathrm{c}}$ evaluated at the equator (Tsurutani and Smith, 1977; Meredith et al., 2001). These waves are supposed to be excited by the anisotropy of the distribution of electrons injected into the inner magnetosphere (Meredith et al., 2001). They can be directly generated with large wave-normal angles (Santolík et al., 2009; Haque et al., 2011) or experience an increase of their wave-normal angle during their propagation away from the source region located near the equator (Lauben et al., 2002; Shklyar et al., 2004; Chum and Santolík, 2005; Bortnik et al., 2011a; Breuillard et al., 2012; Nemec et al., 2012; Chen et al., 2013). Interestingly, recent works have shown that chorus waves may also be the source (or seed) of hiss-type waves inside the plasmasphere (Boskova et al., 1990; Hattori et al., 1991; Bortnik et al., 2011a).

The distribution of $\theta$ angles between wave vector and the background magnetic field as a function of latitude $\lambda$ can be obtained from the data from the STAFF-SA experiment on Cluster (Cornilleau-Wehrlin et al., 2003). Here we present Cluster statistics collected in the range $L=4$ to 5 for lowerband chorus waves with mean frequency $\omega_{\mathrm{m}} \approx 0.35 \Omega_{\mathrm{c} 0}$ in the low to medium geomagnetic activity range (see details of statistics in Agapitov et al., 2012a). The distribution of wave occurrences for given $\theta$ and $\lambda$ is shown in Fig. 1. For each $\lambda$ the total number of wave occurrences are normalized to one. We also show the Gendrin angle $\theta_{\mathrm{g}}(\lambda)$ (Gendrin, 1961) and resonance cone angle $\theta_{\mathrm{r}}(\lambda)$ as functions of $\lambda$. It should be noted that waves cannot be observed above $\theta_{\mathrm{r}}$ in cold plasma theory, although it may be possible in hot plasmas: the corresponding points in this figure are most probably due to uncertainties in the determination of $\theta_{\mathrm{r}}$ (calculated here for $L=4.5 ; \theta_{\mathrm{r}}$ can also differ slightly from $\arccos \left(\omega_{\mathrm{m}} / \Omega_{\mathrm{c}}\right)$ due to the effect of a finite ratio $\Omega_{\mathrm{c}} / \Omega_{\mathrm{pe}}$ at high latitudes, where $\Omega_{\mathrm{pe}}$ is the plasma frequency) or to errors in the determination of $\theta$ (see discussion in Agapitov et al., 2012a). Figures 1-2 show that, at $L \sim 4$ to 5, lower-band chorus waves are rather oblique above $5-10^{\circ}$ of latitude, a large portion of their distribution being located between the Gendrin and resonance cone angles (especially for $\lambda>10^{\circ}$ ). The mean wave-normal angle, as well as the variance, increases with latitude. In addition, one can mention a group of parallel waves observed at high latitudes. This group results in an increase of the variance of the total distribution of $\theta$ angle, $g(\theta)$, but does not change the overall tendency of growth of the mean $\theta$ with $\lambda$. The distribution of wave amplitude $B_{\mathrm{w}}(\theta)$ and its rms value are also displayed in Fig. 2 as a function of wave-normal angle in given latitude ranges. While the maximum amplitudes are generally obtained for moderately oblique waves $\left(\theta \sim 20^{\circ}\right)$, similar levels can also be found between the Gendrin and resonance cone angle on the dayside. The wave power distribution as a function of latitude $B_{\mathrm{w}}^{2}(\lambda)$ has already been provided and fitted by Artemyev et al. (2012b) for dayside waves. Nightside chorus waves are confined at latitudes lower than $20^{\circ}$ but will not be considered here, because we are mostly interested in estimating the actual loss rates of longitudinally drifting trapped electrons, which are principally determined by dayside waves (Shprits et al., 2007). The presented wave-normal distribution is actually representative of the dayside outer-belt region $L=3.5$ to 5.5 for $\mathrm{Kp}<3$. At larger $L>5.5$, lower-band chorus waves are less oblique, although a few percent of the wave power remains at very oblique angles between $10^{\circ}$ and $20^{\circ}$ of latitude. The difference between $L<5.5$ and $L>5.5$ cases is probably related to Landau damping by suprathermal electrons, which has been shown to produce a stronger attenuation of oblique waves at large $L>5.5$ and high latitudes (Bortnik et al., 2006; Chen et al., 2013), although Landau damping is presumably weaker on the dayside than on the nightside ( $\mathrm{Li}$ et al., 2011).

Plasmaspheric whistlers are mainly composed of hiss waves between $100 \mathrm{~Hz}$ and $2 \mathrm{kHz}$ and lightning-generated whistlers between 2 and $5 \mathrm{kHz}$ (Meredith et al., 2007), with an occasional presence of waves from ground-based very low frequency (VLF) transmitters near $20 \mathrm{kHz}$ at $L<2.5$ (Abel and Thorne, 1998). For plasmaspheric whistlers, we make use of Cluster statistics at $L=2$ to 2.5 to derive fits to the wave-normal angle distribution $g(\theta)$. Figure 3 shows the measured wave power distribution as a function of $\theta$ and latitude. While the waves start as quasi-parallel at the equator, they become more oblique at higher latitudes and the variance increases. These observations are in good agreement with ray-tracing results from Bortnik et al. (2011b) (see their Fig. 7), which also showed an increase of hiss wave-normal angles during propagation to higher latitudes, as well as a slight increase of the variance. Very oblique waves represent a significant amount of the distribution for $\lambda>15^{\circ}$. CRRES measurements of amplitudes and spectra between $L=2$ and 3.5 (Meredith et al., 2007, 2009) will be used for lifetime calculations in Sect. 5. Polynomial fits to the average mean and variance of the wave-normal angle obtained from Cluster data will also be used (see Sect. 4). While wave-normal angle satellite data may suffer from some uncertainty, it should be noted that ray-tracing calculations of lightning-generated whistlers have independently shown that their wave-normal angle can be easily larger than $50^{\circ}$ near $L=2$ (Thorne and Horne, 1994).

\section{Analytical estimates of quasi-linear electron lifetimes}

\subsection{Approximate diffusion coefficients}

Oblique whistler waves (Burton and Holzer, 1974; Hayakawa et al., 1990; Tsurutani et al., 2009; Haque et al., 2010; Agapitov et al., 2011; Li et al., 2011; Agapitov et al., 2012b) as well as nearly parallel ones are taken into account in estimates of pitch-angle diffusion rates by considering a double distribution $g(\theta)=g_{\mathrm{s}}(\theta)+g_{1}(\theta)$ containing a 


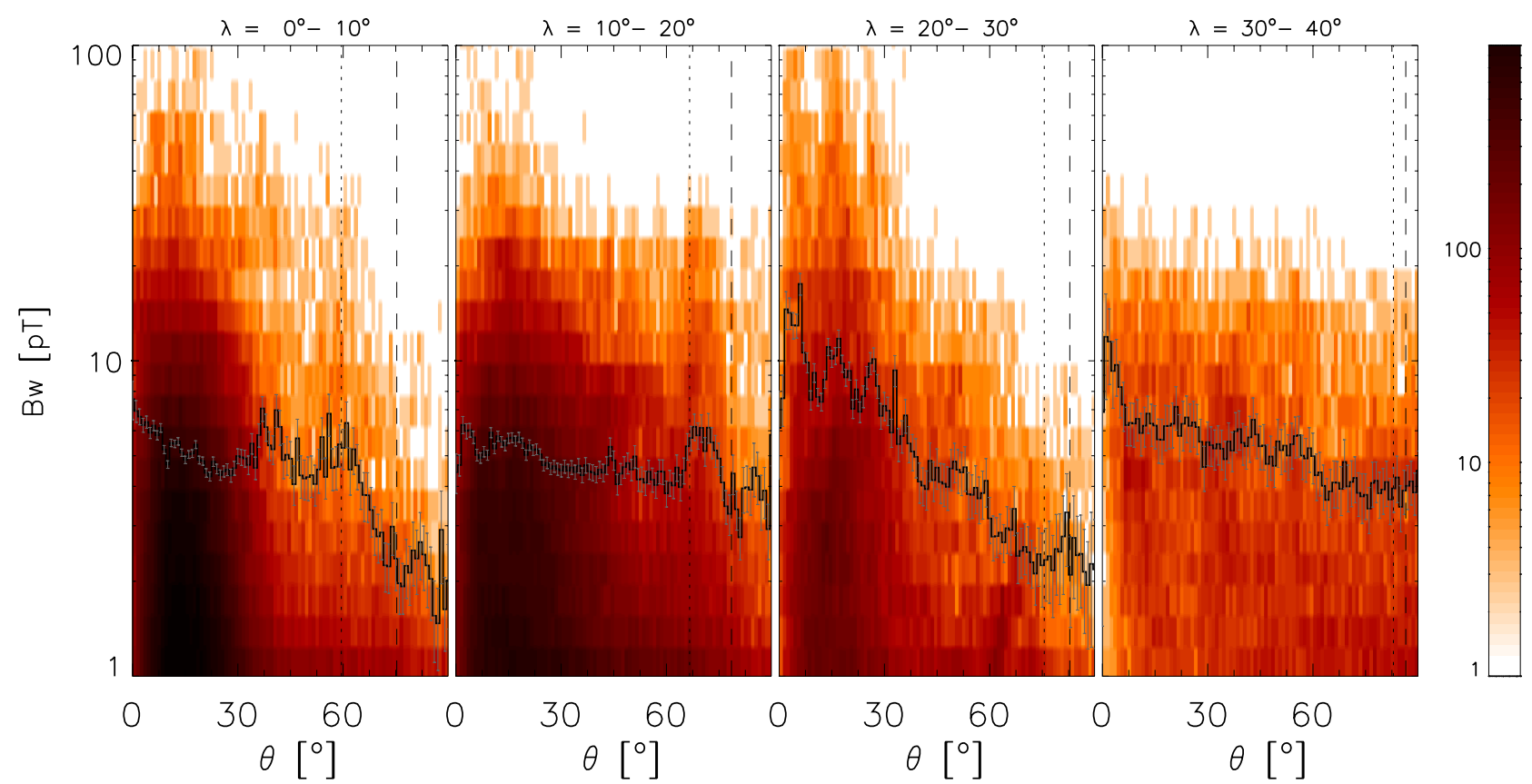

Fig. 2. Distribution of lower-band chorus amplitudes at four ranges of magnetic latitude for $4<L<5$ on the dayside when Kp $<3$. Black curves show rms levels corresponding to average intensity.

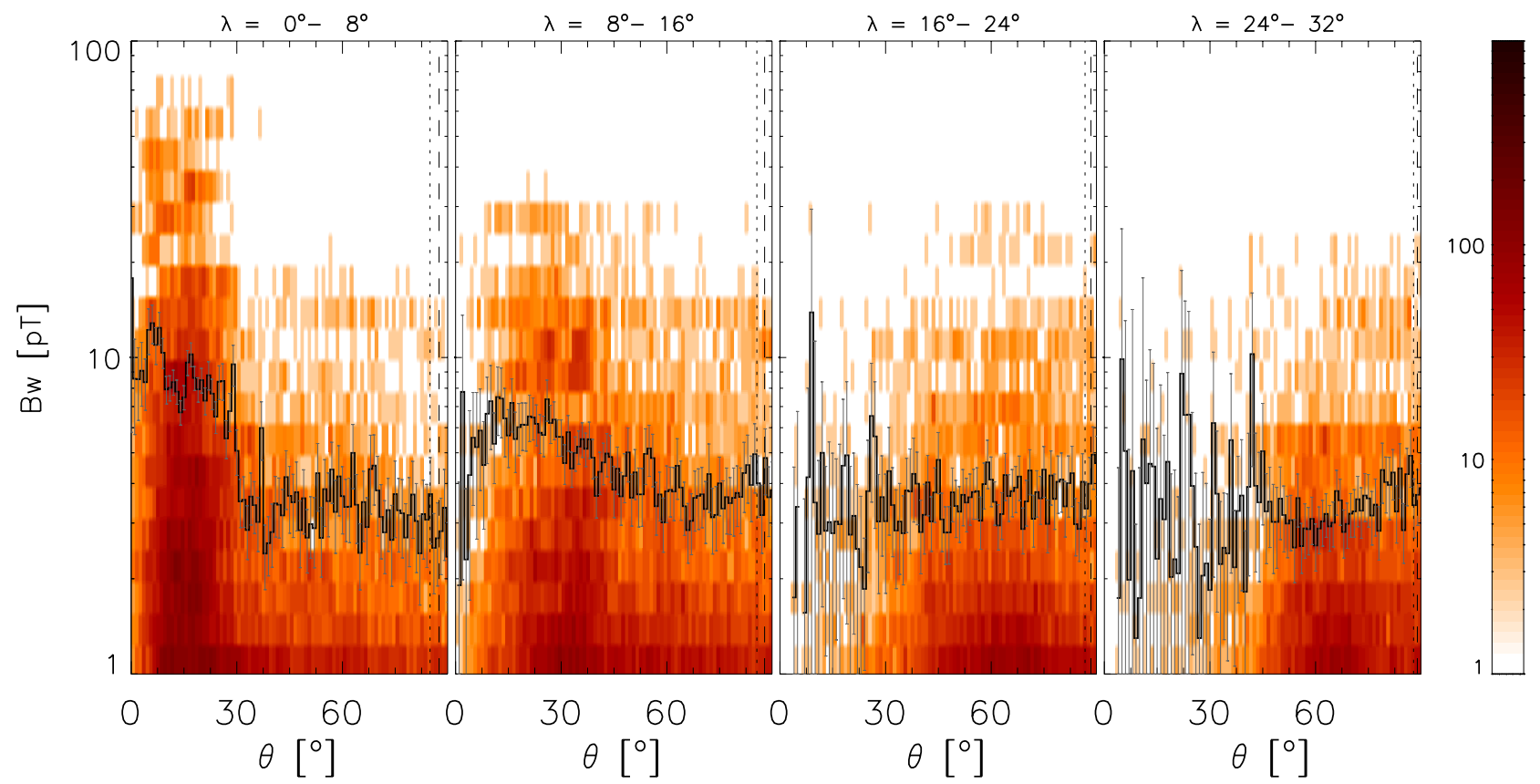

Fig. 3. Distribution of hiss amplitudes in four ranges of magnetic latitude for $2<L<2.5$ on the dayside when Kp $<3$. Black curves show rms levels corresponding to average intensity.

non-Gaussian tail (see Fig. 1 from Mourenas et al., 2012b). The small- $\theta$ part $g_{\mathrm{s}}=\exp \left(-\left(\tan \theta-\tan \theta_{\mathrm{m}}\right)^{2} / \tan ^{2} \Delta \theta\right)$ is assumed to be approximately Gaussian with a width of $\Delta \theta \leq 45^{\circ}$ and a maximum at $\theta_{\mathrm{m}} \approx 0$ (corresponding in practice to $\theta_{\mathrm{m}}<\Delta \theta \leq 45^{\circ}$ ), with lower and upper bounds at $\theta_{\mathrm{lc}}=0$ and $\theta_{\mathrm{uc}} \sim \Delta \theta$. The large- $\theta$ tail is taken as a step function $g_{1}(\theta)$ equal to a constant between the Gendrin and resonance cone angles, being zero otherwise. It can lead to higher electron diffusion rates by allowing higher order cyclotron resonances with whistler waves. 
The local pitch angle quasi-linear diffusion coefficient $D$ of Lyons (1974) (with dimensions of $\mathrm{s}^{-1}$ ) has been conveniently rewritten by Albert $(2005,2007)$ in the form

$$
\begin{aligned}
D & =\frac{D^{\alpha \alpha}}{p^{2}}=\frac{\Omega_{\mathrm{c}}}{\gamma^{2}} \frac{B_{\mathrm{w}}^{2}}{B^{2}} \sum_{n=-\infty}^{+\infty} \sum_{\omega} D_{\mathrm{n}}^{\alpha \alpha} \\
D_{\mathrm{n}}^{\alpha \alpha} & =\int_{\theta_{\min }}^{\theta_{\max }} \sin \theta d \theta \Delta_{\mathrm{n}} G_{\omega} G_{\theta}
\end{aligned}
$$

with

$G_{\theta}(\omega, \theta)=\frac{g(\theta)}{\int_{\theta_{\min }}^{\theta_{\max }} d \theta^{\prime} \sin \theta^{\prime} \Gamma\left(\theta^{\prime}, \omega(\theta)\right) g\left(\theta^{\prime}\right)}$,

where $G_{\omega}\left(\omega(\theta), \theta^{\prime}\right) \sim B_{\mathrm{sp}}^{2}(\omega) / \int B_{\mathrm{sp}}^{2}(\omega) d \omega$ is a function of the wave refractive index. One can find detailed descriptions and analysis of properties of $G_{\omega}$ as well as definitions of $\Delta_{\mathrm{n}}$ and $\Gamma$ in the works by Albert $(2005,2007)$. Here, $B$ is the local magnetic field (of equatorial value $B_{0}$ ), and the argument $x=\left(\omega \gamma / \Omega_{\mathrm{c}}+n\right) \tan \alpha \tan \theta$ of the Bessel functions in $\Delta_{\mathrm{n}}$ is approximated by $x \sim n \tan \alpha \tan \theta$ at low frequency when $\omega \gamma / \Omega_{\mathrm{c}} \ll 1 \leq n$, with $\gamma$ the relativistic factor. The wave spectral density is written as $B_{\mathrm{sp}}^{2}(\omega)=B_{\mathrm{w}}^{2} \exp (-(\omega-$ $\left.\left.\omega_{\mathrm{m}}\right)^{2} / \Delta \omega^{2}\right)$, with $\Delta \omega \approx \omega_{\mathrm{m}} / 2$. In Eq. (1), $G_{\theta}(\omega, \theta)$ and $D_{\mathrm{n}}^{\alpha \alpha}$ are both evaluated at the resonant frequency $\omega$ corresponding to a $\theta$, a harmonic number $n$, and a pitch angle $\alpha$ obtained from the cyclotron resonance condition

$\omega+n \Omega_{\mathrm{c}} / \gamma=k v \cos \theta \cos \alpha$,

where $v$ denotes the electron velocity and $k$ is the wavenumber. Since there may exist several roots of $\omega$, a sum over $\omega$ in Eq. (1) may be necessary. Finally, a bounce average over latitudes has to be performed (Lyons et al., 1972).

For lower-band $\left(\omega_{\mathrm{m}} / \Omega_{\mathrm{c}}<1 / 2\right)$ chorus waves, hiss waves, lightning-generated waves, or VLF waves from transmitters, all of which are right-hand polarized oblique whistlermode waves, the Appleton-Hartree dispersion relation valid for $\omega^{2} \gg \omega_{\mathrm{LH}}^{2}$ ( $\omega_{\mathrm{LH}}$ being the lower-hybrid frequency) can be simplified to $(c k)^{2}=\Omega_{\mathrm{pe}}^{2} \omega /\left(\Omega_{\mathrm{c}} \cos \theta-\omega\right)$ (Helliwell, $1965)$ provided that $\Omega_{\mathrm{pe}}^{2} /\left(\Omega_{\mathrm{c}} \omega\right) \gg 1$ (the high-density limit) and $\sin \theta\left(\Omega_{\mathrm{c}} / \Omega_{\mathrm{c} 0}\right)\left(\Omega_{\mathrm{c} 0} / \Omega_{\mathrm{pe}}\right)<\left(\Omega_{\mathrm{c}} \cos \theta / \omega\right)^{1 / 2}$ (throughout this paper, the subscript " 0 " denotes equatorial values). For moderately oblique, low frequency waves such that $\theta \leq$ $45^{\circ}$ and $\left(\omega / \Omega_{\mathrm{c} 0}\right) \ll \Omega_{\mathrm{pe}}^{2} /\left(12 \Omega_{\mathrm{c} 0}^{2}\right)$, the discrepancy between the exact and approximate resonance frequencies should then remain negligible up to latitudes $\lambda \sim 45^{\circ}$. For $\Omega_{\mathrm{pe}} / \Omega_{\mathrm{c} 0}>4$, it is suitable for all moderately oblique whistler waves satisfying the condition $\omega / \Omega_{\mathrm{c} 0}<0.4$, i.e., lower-band chorus (in agreement with numerical results from Glauert and Horne, 2005), hiss, lightning-generated and VLF transmitters. The lower the frequency ratio $\omega / \Omega_{\mathrm{c} 0}$, the closer the approximate dispersion is to the Appleton-Hartree one. Thus, the approximate dispersion turns out to be almost exact for moderately oblique plasmaspheric whistler waves (hiss and lightninggenerated waves).

For significantly oblique lower-band chorus (or any other kind of low-frequency whistler waves) such that $\theta>\theta_{\mathrm{g}}$, (the Gendrin angle such that $\cos \theta_{\mathrm{g}} \sim 2 \omega_{\mathrm{m}} / \Omega_{\mathrm{c}}$ ), the maximum latitude $\lambda_{\mathrm{MM}}$ for resonance over the whole large- $\theta$ range (from the Gendrin up to nearly the resonance cone angle given by $\cos \theta_{\mathrm{r}} \sim \omega_{\mathrm{m}} / \Omega_{\mathrm{c}}$ ) is reached at small equatorial pitch angles. It has been estimated from the simplified dispersion and resonance condition by Mourenas et al. $(2012 \mathrm{~b}, \mathrm{a})$ as $\lambda_{\mathrm{MM}} \leq 35^{\circ}$ for $L=4$ to 6 , implying that the approximate dispersion can be used only for $\Omega_{\mathrm{pe}} / \Omega_{\mathrm{c} 0}>4$ for $\theta \geq \theta_{\mathrm{g}}$. The discrepancy between the approximate and exact dispersions becomes more important at the higher latitudes, close to the loss-cone edge $\left(\alpha_{0}<10^{\circ}\right)$. Nevertheless, the error in the value of the resonance frequency merely corresponds to a small downward shift from the actual latitude at which resonance occurs at peak wave power, thanks to the rapid increase of $\Omega_{\mathrm{c}}$ at high latitudes. Figure 4 shows that this remains true up to the highest latitudes for $\Omega_{\mathrm{pe}} / \Omega_{\mathrm{c} 0}>4$, while it is still roughly the case for $\Omega_{\mathrm{pe}} / \Omega_{\mathrm{c} 0}=2$ at latitudes smaller than $20^{\circ}$, corresponding to the highest latitude of resonance at $\alpha_{0}>20^{\circ}$ (Mourenas et al., 2012b). After integration over latitudes, this small shift can nevertheless produce large errors in the bounce-averaged diffusion rate $\langle D\rangle_{\mathrm{B}}$ for $\alpha_{0}<10^{\circ}$, due the significant increase with latitude of the integrated function $\Delta_{\mathrm{n}} G_{\theta}$ in Eq. (1) in the large- $\theta$ range at high latitudes $\lambda>20^{\circ}$ (see Appendix A in the work by Mourenas et al., 2012a, and the numerical comparison of the simplified and Appleton-Hartree dispersions in Sect. 5.1). Because peak resonance occurs at higher latitude with the full dispersion, $\langle D\rangle_{\mathrm{B}}$ ends up to be larger for $\alpha_{0}<10^{\circ}$ than with the simplified dispersion. In the simplified analytical model developed by Mourenas et al. (2012b), however, $\langle D\rangle_{\mathrm{B}}$ is only calculated for $\alpha_{0}>15^{\circ}$ and it is simply assumed to continue increasing at least like $1 / \sin \alpha_{0}$ towards the loss cone for $\alpha_{0}<10^{\circ}$ as actually observed in the full numerical simulations (see Sect. 5.1). Since we are principally interested in lifetime estimates, the large- $\langle D\rangle_{B}$ part at small $\alpha_{0}<10^{\circ}$ can then be safely neglected in Eq. (7) below. An error concerning this part should indeed lead to a much smaller relative error in the lifetime estimate. It is this model of $\langle D\rangle_{B}$ (Mourenas et al., $2012 \mathrm{~b}$ ) that will be used throughout the present paper.

As the full expression of the diffusion rate involves four successive integrations of rather complex functions, further approximations have to be made to enable an analytical estimation of the bounce-averaged diffusion rates. The weighted-average reformulation of the local quasi-linear diffusion coefficient introduced by Albert (2007) in his Eq. (7) is first used to simplify the calculations. Such a reformulation is justified by (1) the nearly constant resonant $\omega$ up to $\theta \sim 60-70^{\circ}$ for low-frequency whistler waves using the full dispersion relation (Albert, 2007) and (2) the narrow width $\Delta \omega \leq \omega_{\mathrm{m}} / 2$ of the wave spectrum, which implies that significant contributions to diffusion come principally 


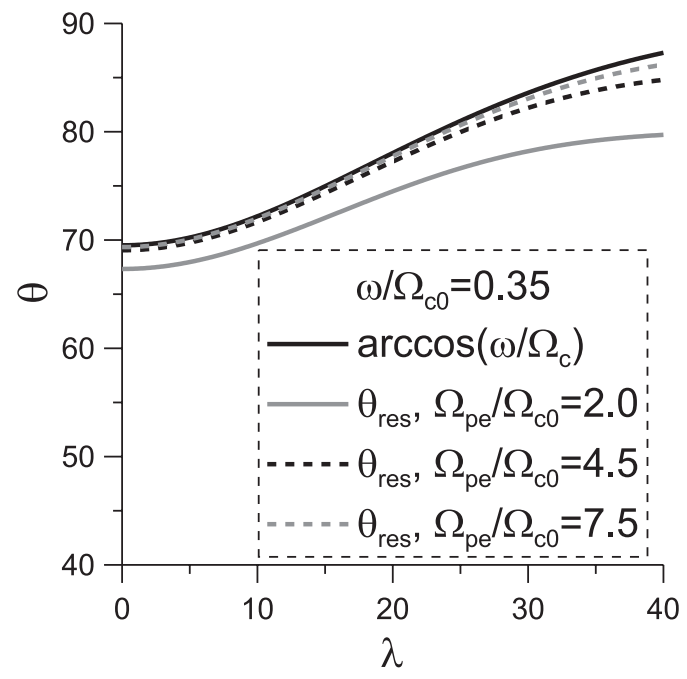

Fig. 4. Resonance cone angle as a function of latitude. The approximate value $\theta_{\mathrm{r}}=\arccos \left(\omega_{\mathrm{m}} / \Omega_{\mathrm{c}}\right)$ from the simplified dispersion relation (solid black line) is compared with exact solutions obtained with the Appleton-Hartree dispersion for different ratios $\Omega_{\mathrm{pe}} / \Omega_{\mathrm{c} 0}$.

from frequencies close to $\omega_{\mathrm{m}}$. It is then acceptable to replace $G\left(\omega\left(\theta^{\prime}\right), \theta\right)$ by $G(\omega(\theta), \theta)$ in Eq. (2), hence allowing the very convenient weighted-average reformulation. Contrary to the mean value approximation proposed by Albert (2007), however, the variation of the averaged function with $\theta$ is taken into account in our analytical calculations, where Bessel functions are replaced by their classic series expansions on both sides of their first maxima (for more details, see Mourenas and Ripoll, 2012; Mourenas et al., 2012b,a). As concerns bounce-averaging, the latitudinal range $\Delta \lambda$ where resonance occurs is evaluated from Eq. (3) and adiabatic invariance for $\omega$ varying between $\omega_{\mathrm{m}}-\Delta \omega$ and $\omega_{\mathrm{m}}+\Delta \omega$. This latitudinal range being narrow and the latitudinal variation of the integrand in the bounce integral remaining weak (see Appendix D and A from Mourenas et al., 2012b,a), the diffusion rate integrated over $\Delta \lambda$ can be further approximated by this integrand taken at $\langle\lambda\rangle$ and $\omega=\omega_{\mathrm{m}}$, multiplied by $\Delta \lambda$ and by $\left\langle B_{\mathrm{sp}}^{2}\right\rangle_{\omega} / B_{\mathrm{sp}}^{2}\left(\omega_{\mathrm{m}}\right)$ (Mourenas et al., 2012b). Finally, it is assumed that the plasma density does not vary strongly with latitude for latitudes smaller than about $40^{\circ}$, in agreement with observations between $L \sim 2$ and 7 (Denton et al., 2006; Ozhogin et al., 2012).

For the small- $\theta$ part $\left(\theta<\theta_{\text {Max }}^{s}<\max \left(\pi / 4, \theta_{\mathrm{g}}\right)\right)$ of the wave-normal angle distribution, the first cyclotron resonance $n=-1$ provides the main contribution to the diffusion rate near the loss-cone edge for distributions such that $g_{\mathrm{s}}\left(\theta_{\mathrm{g}}\right) \ll g_{\mathrm{s}}(0)$ and/or $E \leq 1 \mathrm{MeV}$. This contribution is essentially equivalent to the parallel propagation approximation (PPA) formulated by Summers (2005). At higher equatorial pitch angles, the Landau resonance $n=0$ and higherorder cyclotron resonances may also contribute. For $\theta<45^{\circ}$, the maximum number of significant resonances is $N_{\max }^{s} \sim$ $p \varepsilon_{\mathrm{m} 0} \sin \left(\theta_{\mathrm{Max}}^{s}\right)$ (Mourenas and Ripoll, 2012; Mourenas et al., $2012 \mathrm{~b})$ with $\varepsilon_{\mathrm{m} 0}=\Omega_{\mathrm{pe}} / \Omega_{\mathrm{c} 0} \sqrt{\omega_{\mathrm{m}} / \Omega_{\mathrm{c} 0}}$ and $p$ the electron momentum normalized on $m_{\mathrm{e}} c$. For $\theta>\max \left(\pi / 4, \theta_{\mathrm{g}}\right)$ and at the equator, $\theta$ may take any value from the Gendrin angle up to nearly the resonance angle for resonances $|n| \leq N_{\mathrm{r}}$ with average $N_{\mathrm{r}} \approx 2 \cos \alpha_{0} p \varepsilon_{\mathrm{m} 0} \sqrt{\omega_{\mathrm{m}} / \Omega_{\mathrm{c} 0}}$ (Mourenas et al., $2012 \mathrm{~b}, \mathrm{a}$ ). As the electron leaves the equator and moves towards its mirror point along a magnetic field line, pitch angle increases due to adiabatic invariance, allowing resonance to be recovered at higher latitudes. The total effective number of (positive and negative) contributing resonances is roughly $4 N_{\mathrm{r}}$. Diffusion at large wave-normal angles may increase strongly (partly off the equator) at moderate equatorial pitch angles due to the large number of contributing resonances and owing to the fact that their contributions are roughly independent of $n$.

The full diffusion coefficient is obtained after integration over the bounce motion (Lyons et al., 1972). For the small- $\theta$ part, it yields near the loss-cone (LC) edge with $\alpha_{0} \sim \alpha_{\mathrm{LC}}$ :

$$
\begin{aligned}
\left\langle\left. D_{\mathrm{small}} \alpha_{0}\right|_{\mathrm{B}} ^{s}\right. & \approx \frac{\pi B_{\mathrm{w}}^{2} \Omega_{c 0} \omega_{\mathrm{m}}}{4 \gamma B_{0}^{2} \Delta \omega\left(p \varepsilon_{\mathrm{m} 0}\right)^{13 / 9} T\left(\alpha_{\mathrm{LC}}\right) \cos ^{2} \alpha_{\mathrm{LC}}} \times \\
& \times \frac{\Delta \lambda_{\mathrm{R}, \mathrm{N}}\left(1+3 \sin ^{2} \lambda_{\mathrm{R}}\right)^{7 / 12}(1-\varpi)}{\left|\gamma \varpi-2 \gamma \varpi^{2}+1\right||1-\gamma \varpi|^{4 / 9}},
\end{aligned}
$$

where $\varpi=\omega_{\mathrm{m}} / \Omega_{\mathrm{c}}$ and the bounce period is $T\left(\alpha_{0}\right) \approx 1.38$ $0.64 \sin ^{3 / 4} \alpha_{0}$ (Davidson, 1976). The latitude of resonance $\lambda_{\mathrm{R}}$ for $\omega \approx \omega_{\mathrm{m}}$ can be written as

$\lambda_{\mathrm{R}}^{2} \sim \frac{3}{2}-\frac{3}{2} \sqrt{1-\frac{16}{9}\left(1-\left(\frac{|1-\gamma \varpi|}{p \varepsilon_{\mathrm{m} 0} \sqrt{\cos \Delta \theta}}\right)^{1 / 9}\right)}$

where Eq. (5), which is obtained through a second-order expansion of cosine and sine terms, is sensibly more accurate than the first-order Eq. (C7) in the work by Mourenas and Ripoll (2012) at large density, frequency and energy (i.e., at high latitudes). $\Delta \theta$ denotes the width of the Gaussian distribution $g_{\mathrm{s}}(\theta)$. In Eq. (4), the latitudinal range of resonance corresponding to $\Delta \omega$ is $\Delta \lambda_{R} \sim$ $2\left(\sqrt{\lambda_{\mathrm{R}}^{2}+2 \Delta \omega /\left(27 \omega_{\mathrm{m}}\right) /\left(p \varepsilon_{\mathrm{m} 0}\right)^{1 / 9}}-\lambda_{\mathrm{R}}\right)$ when waves are present up to the highest latitudes (Mourenas et al., 2012b). The actual latitudinal range of resonance can be written as $\Delta \lambda_{R, N} \sim \max \left(\min \left(\lambda^{+}, \lambda_{R}+\Delta \lambda_{R} / 2\right)-\lambda_{R}+\Delta \lambda_{R} / 2,0\right)$ to account for the possible confinement of waves below a certain latitude $\lambda^{+}$, as in the case of chorus (Shprits et al., 2006; Li et al., 2011; Artemyev et al., 2012b). For $\omega_{\mathrm{m}} / \Omega_{\mathrm{c} 0}<0.5$, one can also safely use $\Omega_{\mathrm{c}} / \Omega_{\mathrm{c} 0} \approx\left(p \varepsilon_{\mathrm{m} 0}\right)^{2 / 3}$ in the two above equations (Mourenas et al., 2012b), except in the case when $p \varepsilon_{\mathrm{m} 0}<1$ : then, one must simply take $\lambda_{\mathrm{R}}=0$ and $\Omega_{\mathrm{c}}=\Omega_{\mathrm{c} 0}$. The small- $\theta$ Landau resonance coefficient has been evaluated by Mourenas and Ripoll (2012) for hiss waves and extended to arbitrary ratios $\omega_{\mathrm{m}} / \Omega_{\mathrm{c} 0}<0.5$ by Mourenas et al. (2012b). The lifetime contribution of this Landau term is given explicitly below. 
Albert and Shprits (2009) have shown that lifetimes are determined by the minima of the total diffusion coefficient multiplied by $\tan \alpha_{0}$. As a result, the small- $\theta$ diffusion coefficient is usually the smallest either near the loss-cone angle $\alpha_{\mathrm{LC}}$ or, at large pitch angles, between the Landau and first cyclotron resonance peaks (Shprits et al., 2006, 2007; Albert and Shprits, 2009; Mourenas and Ripoll, 2012). Since our goal is to provide lifetime estimates, we can safely neglect in the small- $\theta$ part all the resonances except for the first cyclotron one at small pitch angles and the Landau and first cyclotron resonances at large pitch angles. The latter indeed define the depth of the minimum in $\langle D\rangle_{\mathrm{B}}$ at large pitch angles (see next section).

For the more complicated large- $\theta$ part of $g(\theta)$, such that $\theta \geq \max \left(\pi / 4, \theta_{\mathrm{g}}\right)$, lifetimes are mainly determined by the moderate to large pitch-angle region where diffusion is weakest. Then, many resonances $n$ contribute to diffusion. The details of the derivation of $D$ at pitch angles larger than $20^{\circ}$ are given in Mourenas et al. (2012b). With $\theta$ bounds taken as $\theta_{\mathrm{g}}$ and $\theta_{\mathrm{r}}$ and $\Delta \omega / \omega_{\mathrm{m}} \sim 0.5$, one gets

$$
\langle D\rangle_{\mathrm{B}}^{l}=\frac{\left(1+\frac{\omega_{\mathrm{m}}^{2}}{\Omega_{\mathrm{c} 0}^{2}}\right) B_{\mathrm{w}}^{2} \Omega_{\mathrm{c} 0} \sqrt{8} N_{\mathrm{r}}}{9 \gamma B_{0}^{2}\left(p \varepsilon_{\mathrm{m} 0}\right)^{2} \cos \alpha_{0} \sin \alpha_{0}}
$$

where the maximum latitude of resonance is generally smaller than $35^{\circ}$ (Mourenas et al., 2012b,a), i.e., smaller than the latitude upper bound on intense oblique waves $\lambda^{+} \sim$ $40^{\circ}$ in the dayside outer belt at $L<5.5$ (see Sect. 2). On the nightside or at large $L>5.5$, the presence of oblique chorus is limited at $\lambda<\lambda_{\mathrm{Obl}} \sim 20^{\circ}$ due to strong Landau damping by suprathermals (Bortnik et al., 2006; Li et al., 2011). From Eqs. (7)-(8) from Mourenas et al. (2012a) with $\Delta \lambda \approx \lambda_{\mathrm{Max}} / 2 \approx \lambda_{\mathrm{Obl}} / 3,\langle D\rangle_{\mathrm{B}}^{l}$ becomes much smaller than the value (Eq. 6) for $\sin \alpha_{0}<\left(\omega_{\mathrm{m}} / \Omega_{\mathrm{c} 0}\right)\left(\Omega_{\mathrm{c} 0} / \Omega_{\mathrm{c}}\left(\lambda_{\mathrm{Obl}}\right)\right)^{3 / 2}$. It leads to a threshold at $\alpha_{0}<\alpha_{\mathrm{Obl}}=\omega_{\mathrm{m}} / \Omega_{\mathrm{c} 0}$ (see also Appendix B from Mourenas et al., 2012b) where $\langle D(n)\rangle_{\mathrm{B}}^{l}$ decreases fast like $\left(\sin \alpha_{0} / \sin \alpha_{\mathrm{Obl}}\right)^{2|n|-1}$ until it becomes even smaller than $\langle D\rangle_{\mathrm{B}}^{S}$ near the loss-cone edge for $L>4$.

\subsection{Lifetime expressions for multiple wave modes}

We make use of the approximate reformulation of the electron lifetime by Albert and Shprits (2009), which reads as

$\tau \approx \sigma \int_{\alpha_{\mathrm{LC}}}^{\pi / 2} \frac{\cos \alpha_{0}}{2\langle D\rangle_{\mathrm{B}} \sin \alpha_{0}} d \alpha_{0}$

The numerical coefficient $\sigma \approx 1$ allows recovering precisely numerical simulations: one can take $\sigma \sim 1 / 2$ when the minimum of $\langle D\rangle_{\mathrm{B}}$ is near the loss cone or else when a minimum occurs at large pitch angles but remains moderately deep (see Appendix A), while $\sigma \sim 1$ is more appropriate for the Landau part at high pitch angles (Albert and Shprits,
2009; Mourenas and Ripoll, 2012). A reasonable way to derive an analytical estimate of the lifetimes consists in neglecting the pitch-angle domain wherein $\langle D\rangle_{\mathrm{B}} \tan \alpha_{0}$ is large. Accordingly, the large- $\theta$ part $\langle D\rangle_{\mathrm{B}}^{l}$ can be integrated analytically between about $\pi / 12$ and $\pi / 2$, while the small $\theta$ part $\left\langle D_{\text {small } \alpha_{0}}\right\rangle_{\mathrm{B}}^{s}$ can be integrated analytically (with $T\left(\alpha_{0}\right) \sim$ $\left.T\left(\alpha_{\mathrm{LC}}\right) \sim 1.3\right)$ from the loss-cone angle $\alpha_{\mathrm{LC}}$ up to an angle $\alpha_{+} \approx \max \left(2.2 \alpha_{\mathrm{LC}}, \min \left(7 \alpha_{\mathrm{LC}}, \pi / 2-\theta_{\mathrm{Max}}^{s}\right)\right.$ (the angle $\pi / 2-$ $\theta_{\text {Max }}^{s}$ represents the smallest $\alpha_{0}$ for resonance at the equator - see details in Mourenas and Ripoll, 2012; Mourenas et al., 2012 b). At low energy or $L$, however, a deep minimum of $\langle D\rangle_{\mathrm{B}}$ may occur between the peaks of first cyclotron and Landau resonances, which can strongly increase lifetimes. Its effect can be easily evaluated by integrating in Eq. (7) the Landau diffusion coefficient $\left\langle D_{n=0}\right\rangle_{\mathrm{B}}^{s}$ between the Landau peak and the upper limit of the first cyclotron resonance diffusion coefficient $\left\langle D_{n=-1}\right\rangle_{\mathrm{B}}^{S}$.

For the sake of generality, we hereafter extend our previous model (Mourenas and Ripoll, 2012; Mourenas et al., $2012 \mathrm{~b}$ ) to consider a spectrum consisting of two or three Gaussians of peak frequencies $\omega_{\mathrm{m}, i}$ and upper cutoffs $\omega_{\mathrm{UC}, i}<\omega_{\mathrm{m}, i}+2 \Delta \omega_{\mathrm{m}, i}$, corresponding to different kinds of whistler waves. The $i=1$ index is also assumed to correspond to the highest intensity waves, which are moreover supposed to have the smallest mean and upper cutoff frequencies. Such a situation is actually representative of typical whistler spectra inside the plasmasphere as well as in the outer belt. Inside the plasmasphere, the $i=1$ index would correspond to hiss waves (Meredith et al., 2007, 2009). For simplicity, the remaining whistler modes (e.g., lightninggenerated and VLF) are also classified in the same way, such that a larger index $i$ corresponds to a smaller intensity and higher (mean and upper cutoff) frequencies.

Then, the different cyclotron and Landau diffusion coefficients (corresponding to different indices $i$ ) take on a shape shown schematically in Fig. 5 for $L<3.5$. Cyclotron diffusion by lower-frequency, higher-intensity waves (i.e., hiss with $i=1$ ) dominates at small $\alpha_{0}<\alpha_{\mathrm{UC}, 1}$, followed at higher $\alpha_{0}$ by cyclotron diffusion by lightning-generated waves $(i=2)$, and so on. Finally, cyclotron diffusion drops down at $\alpha_{0}>\max \left(\alpha_{\mathrm{UC}, i}\right)$, and Landau diffusion then takes over. As can be seen in Fig. 5, the total diffusion coefficient can then be approximated over each successive $\alpha_{0}$-range by one dominant individual diffusion coefficient corresponding to the relevant wave index $i$, allowing the integration in Eq. (7) to be performed by parts (see below).

While actual fits to the measured spectra contain sometimes Gaussians that do not follow the above-assumed classification (Meredith et al., 2007), it is always possible to replace them by Gaussians that do comply, even if the corresponding approximation to the actual spectrum is slightly less accurate. In the expression for $\alpha_{+}$, one has then $\theta_{\text {Max }}^{s}=$ $\theta_{\text {Max }, i=1}^{s}$ corresponding to $i=1$ waves. All the small- $\theta$ waves are assumed to have a Gaussian wave-normal distribution $g_{\mathrm{s}, i}(\theta)=\exp \left(-\tan ^{2} \theta / \tan ^{2} \Delta \theta_{i}\right)$, where $\Delta \theta_{i}<45^{\circ}$ is 


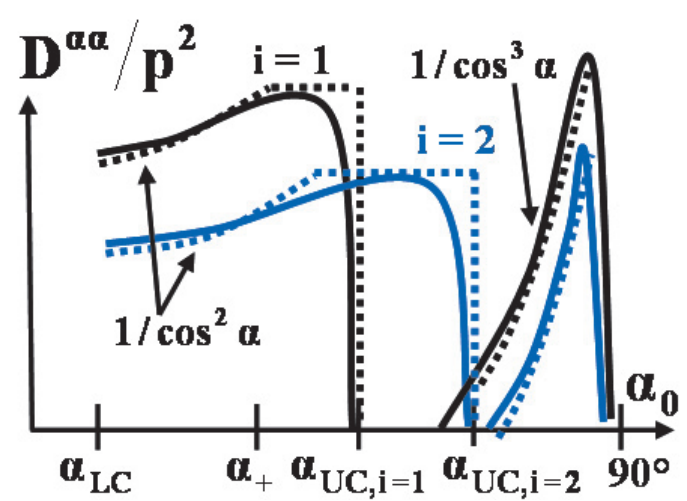

Fig. 5. Schematic representation of the cyclotron and Landau diffusion coefficients for waves $i=1$ (hiss, solid black line) and $i=2$ (lightning-generated, solid blue line) at $L<3.5$, as a function of equatorial pitch angle. The corresponding approximate analytical diffusion coefficients are also displayed (dotted lines).

the characteristic width of the distribution. Further assuming that $\cos \alpha_{0 i}=\left(p \varepsilon_{\mathrm{m} 0, i}\right)^{-1}<\sin \Delta \theta_{i}$, Eq. (30) in the work by Mourenas and Ripoll (2012) giving the cyclotron diffusion rate at large equatorial pitch angles simplifies to

$\left\langle D_{n=-1, i, \operatorname{large} \alpha_{0}}\right\rangle_{\mathrm{B}}^{s}=\frac{B_{\mathrm{w}, i}^{2} \Omega_{\mathrm{c} 0}}{\sqrt{3} \gamma B_{0}^{2} p \varepsilon_{\mathrm{m} 0, i} \sin \alpha_{0 i} \tan \Delta \theta_{i}}$.

Posing $\quad \cos \alpha_{\mathrm{UC}, i}=\min \left(\left(p \varepsilon_{\mathrm{UC} 0, i}\right)^{-1}, 1\right) \quad$ (with $\quad \varepsilon_{\mathrm{UC} 0, i}=$ $\left.\varepsilon_{\mathrm{m} 0, i} \sqrt{\omega_{\mathrm{UC}, i} / \omega_{\mathrm{m}, i}}\right)$, the small- $\theta$-waves-only lifetime can be written as

$\tau_{\mathrm{S}} \approx \tau_{\text {Landau }}+\frac{\ln \left(\sin \alpha_{+} / \sin \alpha_{\mathrm{LC}}\right)}{4\left\langle D_{\text {small }} \alpha_{0}, i=1\right\rangle_{\mathrm{B}}^{s}}+\sum_{i=2,3} \Delta \tau_{i}$,

where

$$
\begin{aligned}
\tau_{\text {Landau }} & \approx \frac{B_{0}^{2}\left|1-\omega_{\mathrm{m}, 1} / \Omega_{\mathrm{c} 0}\right|^{-3 / 2} F_{\mathrm{hl}}}{3 \gamma B_{\mathrm{w}, 1}^{2} \Omega_{\mathrm{c} 0}}\left(\frac{p \Omega_{\mathrm{pe}}}{\Omega_{\mathrm{c} 0}}\right)^{4} \frac{\tan \Delta \theta_{i=1}}{\sin ^{3} \alpha_{\mathrm{M} 0,1}} \\
& \times \frac{\frac{\pi}{2}-\alpha_{0}^{\mathrm{Max}}-\frac{2}{3} \sin \left(2 \alpha_{0}^{\operatorname{Max}}\right)-\frac{1}{12} \sin \left(4 \alpha_{0}^{\operatorname{Max}}\right)}{g_{\mathrm{s}}\left(\theta_{\mathrm{M} 0,1}\right)+\min \left(C_{i=1}^{3} / 11, C_{i=1}^{-1}\right)},
\end{aligned}
$$

where $\quad C_{i=1}=p \varepsilon_{\mathrm{m} 0,1} \tan \Delta \theta_{i=1} \quad$ and $\quad \alpha_{0}^{\mathrm{Max}}=$ $\max \left(\alpha_{\mathrm{UC}, i}, \alpha_{\mathrm{LC}}\right)$ are the highest equatorial pitch angles where (first) cyclotron resonance exists. $\tan \theta_{\mathrm{M} 0,1} \approx 1.84 / p \varepsilon_{\mathrm{m} 0, i=1}$ is the wave-normal angle at peak equatorial Landau resonance, and $\cos \alpha_{\mathrm{M} 0,1} \approx\left|1-\omega_{\mathrm{m}, 1} / \Omega_{\mathrm{c} 0}\right|^{1 / 2} \gamma \omega_{\mathrm{m}, 1} /\left(\Omega_{\mathrm{c} 0} p \varepsilon_{\mathrm{m} 0,1}\right) \quad$ corresponds to the position of the Landau peak. The multiplicative term $F_{\mathrm{hl}} \approx \max \left(10 \Theta\left(\pi / 4-\alpha_{0}^{\mathrm{Max}}\right), 1\right)$ with Heaviside function $\Theta(\ldots)$ is such that $F_{\mathrm{hl}}=1$, except when cyclotron resonance becomes unavailable at moderate equatorial pitch angles for low $L$ inside the plasmasphere, as discussed in Appendix B. It is also assumed here that the index $i=1$ corresponds to the highest value of $B_{\mathrm{w}, i}^{2}$ multiplied by
$g_{\mathrm{s}}\left(\theta_{\mathrm{M} 0, \mathrm{i}}\right)+\min \left(C_{i}^{3} / 11, C_{i}^{-1}\right)$ so that the $i=1$ waves dominate in the Landau part of $\tau_{s}$. This condition is generally satisfied for typical plasmaspheric whistler spectra (e.g., see Fig. 2 from Meredith et al., 2007). The filling of the trough in pitch-angle diffusion rate between the Landau and first cyclotron peaks from the $i=1$ waves, which is provided by the $i=2$ and 3 waves, can be written from Eqs. (7)-(8) as

$$
\begin{aligned}
\Delta \tau_{i} & \sim \int_{\alpha_{\mathrm{UC}, i-1}}^{\alpha_{\mathrm{UC}, i}} \frac{\left(1 / \tan \alpha_{0}\right)}{2\left\langle D_{\left.n=-1, i, \operatorname{large} \alpha_{0}\right\rangle_{\mathrm{B}}^{s}}\right.} d \alpha_{0} \\
& \approx \frac{\left(\sin \alpha_{\mathrm{UC}, i}-\sin \alpha_{\mathrm{UC}, i-1}\right)}{2\left\langle D_{\left.n=-1, i, \operatorname{large} \alpha_{0}\right\rangle_{\mathrm{B}}^{s}(\pi / 2)}\right.}
\end{aligned}
$$

Note that in a case where $p \varepsilon_{\mathrm{UC}, i}<2^{1 / 4}$, such as for hiss waves at $L<2.5$ and low energy electrons, things become a little bit more complicated. Then, the $i=1$ index must be given to the first Gaussian such that this inequality is reversed, except for the Landau term in Eq. (9).

Concerning the case of small- and large- $\theta$ distributions, typical of outer belt chorus spectra, the $D$ contributions in Eq. (6) from different large- $\theta$ Gaussian frequency spectra are roughly independent of $\omega_{\mathrm{m}, i}$ and therefore add up into one single term $\langle D\rangle_{\mathrm{B}}^{l}$, with $B_{\mathrm{w}}^{2}=\sum B_{\mathrm{w}, i}^{2}$ with $\omega_{\mathrm{m}}$ taken as the intensity-weighted mean of $\omega_{\mathrm{m}, i}$. Assume that the large- $\theta$ part of the wave distribution represents between $5 \%$ and $50 \%$ of the total wave power, as chorus observations at $L=4$ to 5.5 suggest (Burton and Holzer, 1974; Agapitov et al., 2012b). Then, the relative weights of small- and large- $\theta$ parts in $D$ are made roughly similar by their weighting factors $g(\tan \theta) \sqrt{1+\tan ^{2} \theta} \tan \theta$, so that $D_{\text {total }} \approx\left(D^{s}+D^{l}\right) / 2$ (Mourenas et al., 2012b). Finally, one gets the following from Eqs. (4)-(6) for the total $g(\theta)$ distribution:

$$
\tau_{s+l} \approx 2 \tau_{\text {Landau }}+\frac{0.5}{\langle D\rangle_{\mathrm{B}}^{l}(\pi / 4)+\left\langle D_{\text {small } \alpha_{0}, i=1}\right\rangle_{\mathrm{B}}^{s}}
$$

where one has now $\sin \alpha_{0}^{\mathrm{Max}}=\max \left(\sin \alpha_{\mathrm{UC}, i}\right.$, $\left.\left(2 \omega_{\mathrm{m}, 1} / \Omega_{\mathrm{c} 0}\right), \sin \alpha_{\mathrm{sp}}\right)$ in the expression in Eq. (10) for $\tau_{\text {Landau }}$ with $F_{\mathrm{hl}} \sim 1$ and

$\cos \alpha_{\mathrm{sp}}=\sqrt{\frac{\Omega_{\mathrm{c} 0}}{4 \omega_{\mathrm{UC}, 1}}}\left|\frac{\max \left(0,1-\frac{\gamma \omega_{\mathrm{UC}, 1}}{\Omega_{\mathrm{c} 0}}\right)}{p \varepsilon_{\mathrm{m} 0, i=1} \sqrt{\omega_{\mathrm{UC}, 1} / \omega_{\mathrm{m}, 1}}}\right|$.

In a regime of interaction with weakly oblique low-frequency whistler waves, electron lifetimes given by Eq. (9) scale roughly as

$\tau_{s} \sim \frac{110}{B_{\mathrm{w}}^{2}} \frac{p^{3 / 2} \gamma \omega_{\mathrm{m}, 1}^{7 / 9} \Omega_{\mathrm{pe}}^{14 / 9}}{\Omega_{\mathrm{c} 0}^{12 / 9}} \ln \left(\frac{\sin \alpha_{+}}{\sin \alpha_{\mathrm{LC}}}\right)$

at high enough energy and/or density such that $p \varepsilon_{\mathrm{m} 0}>2^{1 / 4}$ for cyclotron resonance to prevail (see Appendix B and 
Mourenas et al., 2012b). Note that expression in Eq. (14) should be multiplied by a factor of $\sim 2 / 3$ for $\omega_{\mathrm{m}} / \Omega_{\mathrm{c} 0}<$ 0.05 . Here $\tau_{\mathrm{s}}$ is given in seconds and the average (rms) wave amplitude $B_{\mathrm{w}}$ in pT. With a ratio $\omega_{\mathrm{m}, 1} / \Omega_{\mathrm{c} 0}$ fixed, lifetimes due to interaction with lower-band chorus then vary roughly like $E^{2} / L$ at high energies $E$ and/or $L$. It is worth noting that lifetimes $\tau_{\mathrm{s}}$ in Eq. (9) are independent of $\Delta \omega$ and $\Delta \theta$, varying like $\tan \Delta \theta\left(\omega_{\mathrm{m}, 1} / \omega_{\mathrm{UC}, 1}\right)^{5 / 2}$ only at very low energies when a deep minimum in diffusion shows up beside the Landau peak where cyclotron resonance is not available anymore.

When large- $\theta$ whistler waves are present up to high latitudes and at high enough energy to get $\tau_{s+l} \ll \tau_{s}$, Eq. (12) yields

$\tau_{s+l} \sim \frac{35}{B_{\mathrm{w}}^{2}} \frac{\gamma p \Omega_{\mathrm{pe}}}{1+\frac{\gamma \Omega_{\mathrm{c} 0}}{2 p \Omega_{\mathrm{pe}}}}$,

where $\tau_{s+l}$ is given in seconds, $B_{\mathrm{w}}$ in pT, and the additional term at the denominator stems from a slightly more accurate expression of $N_{\mathrm{r}}$ at very low energy and density given in Eq. (7) from Mourenas et al. (2012b).

Comparing Eqs. (15) and (14), one finds that $\tau_{s+l} / \tau_{s}<1$ to $\ll 1$. The eventual reduction of lifetimes brought forth by the presence of very oblique whistler waves turns out to be more important for higher values of $\Omega_{\mathrm{pe}} / \Omega_{\mathrm{c} 0}$, i.e., at larger $L$ and higher energy where $\tau_{s+l} \sim \gamma p \Omega_{\mathrm{pe}}$. This reduction of lifetimes stems from the related increase of the number of large- $\theta$ cyclotron resonances $N_{\mathrm{r}}$ at high energy. In the night sector of the outer belt or at $L>5.5$, a neat latitude confinement of very oblique waves occurs due to strong Landau damping (Bortnik et al., 2006; Li et al., 2011) with $\lambda \leq \lambda_{\text {Obl }} \sim 20^{\circ}$. The actual lifetime in Eq. (7) is then roughly determined by small- $\theta$ diffusion between the loss-cone angle $\alpha_{\mathrm{LC}}$ and $\alpha_{+, \mathrm{Obl}} \sim \omega_{\mathrm{m}} / 2 \Omega_{\mathrm{c} 0}=\alpha_{\mathrm{Obl}} / 2$ where large- $\theta$ diffusion steeply increases (see the discussion below Eq. 6).

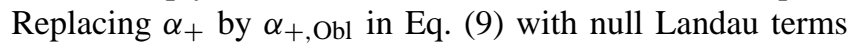
gives an estimate of the lifetime for oblique lower-band chorus waves strictly confined to low latitudes; corresponding lifetimes are typically two times smaller than the small- $\theta$ only lifetime in Eq. (14).

\section{Numerical scheme of calculation of the diffusion coef- ficients and lifetimes}

To calculate diffusion coefficients and estimate lifetimes, we use the approach proposed by Albert (2005) and Glauert and Horne (2005) with modifications corresponding to the dependence of $g(\theta)$ distribution on $\lambda$ (see Artemyev et al., 2012a) and the dependence of the wave amplitude $B_{\mathrm{w}}$ on $\lambda$ (see Artemyev et al., 2012b). However, in contrast to our previous calculations, we use here the Appleton-Hartree dispersion relation (Helliwell, 1965) valid for arbitrary values of the ratio

$$
\begin{array}{r}
\Omega_{\mathrm{pe}} / \Omega_{\mathrm{c}}: \\
(k c / \omega)^{2}=1-\frac{\mathrm{X}(1-\mathrm{X})}{1-\mathrm{X}-\frac{1}{2} \Upsilon \sin ^{2} \theta \pm \sqrt{D}} \\
D=\left(\frac{1}{2} \Upsilon \sin ^{2} \theta\right)^{2}+(1-\mathrm{X})^{2} \Upsilon \cos ^{2} \theta \\
\Upsilon=\Omega_{\mathrm{c}}^{2} / \omega^{2}, \quad \mathrm{X}=\Omega_{\mathrm{pe}}^{2} / \omega^{2} .
\end{array}
$$

For $\mathrm{X} \rightarrow \infty$ one can obtain the simplified dispersion relation $(k c / \omega)^{2}=1-\mathrm{X} /(1 \pm \sqrt{\Upsilon} \cos \theta)$. Solving the system consisting of dispersion relation in Eq. (17) and resonant condition in Eq. (3) gives resonant frequencies and wavenumbers.

\subsection{Outer-belt lower-band chorus waves}

We use a distribution function $g(X)$ with $X=\tan \theta$ of wave occurrences depending on $\lambda: g(X)=\exp \left(-\left(X-X_{\mathrm{m}}\right)^{2} / X_{\mathrm{w}}^{2}\right)$, where $X_{\mathrm{m}, \mathrm{w}}=X_{\mathrm{m}, \mathrm{w}}(\lambda)$ are determined from Cluster observations and approximated by polynomial functions (see Artemyev et al., 2012a). The recent corrections to Cluster STAFF data (see Agapitov et al., 2012b) slightly change the approximations for $X_{\mathrm{m}, \mathrm{w}}(\lambda)$ in comparison with Agapitov et al. (2011) for $L=3.5$ to 5.5 :

$X_{\mathrm{m}}=0.33+0.36 l-0.23 l^{2}+0.086 l^{3}$

$X_{\mathrm{w}}=\sqrt{2}\left(0.43+0.79 l-0.52 l^{2}+0.14 l^{3}\right)$,

where $l=\lambda / 10^{\circ}$ and a latitude upper bound on the presence of intense chorus waves $\lambda<\lambda^{+}=40^{\circ}$ is postulated in rough agreement with observations (Bunch et al., 2012; Artemyev et al., 2012b). This latitude upper cutoff presents the additional advantage that the condition $\omega>\omega_{\mathrm{LH}}$ is satisfied everywhere as required. A comparison of the approximation in Eq. (17) with the previous one (Artemyev et al., 2012a) is shown in Fig. 6. The increase of the mean value $X_{\mathrm{m}}$ with latitude corresponds to very oblique wave propagation and results in intensification of higher order cyclotron harmonic interaction (see, e.g., Shklyar and Matsumoto, 2009) yielding an increase of pitch-angle diffusion (Shprits and Ni, 2009; Ni et al., 2011; Artemyev et al., 2012a). At higher $L>5.5$, the chorus wave-normal angle distribution is less oblique, so that the actual electron lifetimes should be comprised between the values obtained with the above-given oblique distribution valid for $L<5.5$ and with a quasi-parallel distribution. Therefore, we shall also provide below lifetimes calculated for a quasi-parallel distribution of lower-band chorus waves.

We perform most calculations for a constant mean amplitude $B_{\mathrm{w}}=100 \mathrm{pT}$ of the waves. However, the increase of the wave-normal angle $\theta$ with $\lambda$ results in a progressive transformation of whistler waves from an electromagnetic mode near the equator to a quasi-electrostatic mode at very high latitudes (see, e.g., Ginzburg and Rukhadze, 1975; Sazhin and Horne, 1990). As a result, $B_{\mathrm{w}}$ should depend on $\lambda$. We take into account this dependence by using Cluster observations of the distribution of wave amplitudes for each $\lambda$. As a result, we calculate diffusion coefficients and estimate lifetimes for 


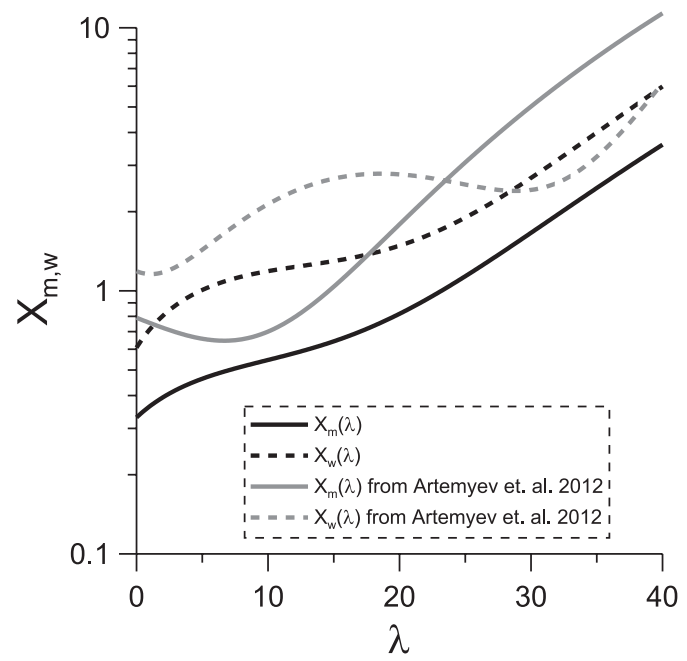

Fig. 6. Mean value $X_{\mathrm{m}}$ and variance $X_{\mathrm{w}}$ from Eq. (17) are shown by black color, while approximations from Artemyev et al. (2012a) are shown by grey color.

various probability levels of $B_{\mathrm{w}}$ distribution depending on $\lambda$ (see details in Artemyev et al., 2012b). Besides, the magnetic amplitude of very oblique waves becomes smaller than the amplitude of quasi-parallel waves in Cluster statistics at high latitudes (see Sect. 2). To quantify this effect, some lifetime calculations will also be performed taking into account the measured variation of $B_{\mathrm{w}}(\theta)$ in the day sector for $3.5<L<5.5$ : the wave power at $\lambda>20^{\circ}$ is then simply divided by 2 to 4 for $\theta>60^{\circ}$.

Although the effects of a non-dipolar magnetic field could be important in the nightside region for large $L \geq 6$ (see, e.g., Orlova and Shprits, 2010; Ni et al., 2011; Ma et al., 2012; Ni et al., 2012; Orlova et al., 2012), we restrict our considerations to a dipole field $B=B_{0} \sqrt{1+3 \sin ^{2} \lambda} / \cos ^{6} \lambda$ for averaging diffusion coefficients over electron bounce oscillations (Lyons et al., 1972). To estimate lifetimes, we use the expression in Eq. (7). For numerical calculations of diffusion coefficients for chorus waves observed in $L \sim 4$ to 5.5, we use $\omega_{\mathrm{m}}=0.35 \Omega_{\mathrm{c} 0}$, a variance $\Delta \omega=0.15 \Omega_{\mathrm{c} 0}$, and a maximum frequency variation of $1.5 \Delta \omega$.

\subsection{Plasmaspheric hiss and lightning-generated waves $(2 \leq L \leq 3)$}

We shall consider two cases at $L=2$ and 2.5 in quiet conditions, as provided by Meredith et al. (2007). For $L=2$ and 2.5 , one has $\Omega_{\mathrm{pe}} / \Omega_{\mathrm{c} 0}=5.6$ and 7.3 , respectively. The $G_{\omega}$ functions are obtained by roughly fitting the corresponding CRRES average spectra, and they will be compared with the different fits given by Meredith et al. (2007) in Sect. 5.6. Here we use two Gaussians for hiss and lightning-generated waves in $G_{\omega}$. In addition, these Gaussians are required to be not-too-wide in order for related analytical estimates to be valid. Approximating CRRES data at $L=2$, one gets $\omega_{\mathrm{m}, 1} \sim$
Table 1. Wave-normal angle models for the different wave modes.

\begin{tabular}{lc}
\hline Wave mode & Wave-normal model \\
\hline Hiss, lightning & Eq. (18) for realistic distribution \\
Chorus & $X_{\mathrm{m}}=0$ and $X_{\mathrm{W}}=1$ for quasi-parallel waves \\
& Eq. (17) for realistic distribution \\
& $X_{\mathrm{m}}=0$ and $X_{\mathrm{w}}=1$ for quasi-parallel waves \\
\hline
\end{tabular}

$(2 \pi) 350$ and $\Delta \omega_{\mathrm{m}, 1}=(2 \pi) 350$ (in $\mathrm{rads} \mathrm{s}^{-1}$ ) and $\omega_{\mathrm{m}, 2} \sim$ $(2 \pi) 2000$ and $\Delta \omega_{\mathrm{m}, 2}=(2 \pi) 2000$ (in rads s ${ }^{-1}$ ) (with cutoffs at $\omega_{\mathrm{m}} \pm 2 \Delta \omega$ in numerical calculations) for $23 \mathrm{pT}$ hiss and 3 pT lightning waves. At $L=2.5$, one gets $\omega_{\mathrm{m}, 1} \sim(2 \pi) 350$ and $\Delta \omega_{\mathrm{m}, 1}=(2 \pi) 300$ (in rads s${ }^{-1}$ ) and $\omega_{\mathrm{m}, 2} \sim(2 \pi) 2000$ and $\Delta \omega_{\mathrm{m}, 2}=(2 \pi) 1700$ (in rads s${ }^{-1}$ ) for $38 \mathrm{pT}$ hiss and $5.5 \mathrm{pT}$ lightning-generated waves. It is clear from our preceding analytical estimates (Mourenas et al., 2012b) that very low frequency hiss wave-normal angles reach the Gendrin angle only above $\theta \sim 88^{\circ}$. On the other hand, lightninggenerated and magnetospherically reflected whistlers have a much higher frequency than hiss (about $3 \mathrm{kHz}$ ), allowing the Gendrin angle to be reached above $85^{\circ}$. A sensible portion of their power is believed to propagate at such oblique angles (Meredith et al., 2007), as it is also the case for VLF transmitters waves near $20 \mathrm{kHz}$, which may already reach the Gendrin angle at $\theta>45^{\circ}$. Since the validity of our numerical and analytical models requires that $\omega>\omega_{\mathrm{LH}}$, the upper bound on latitude integration is limited accordingly, depending on the mean frequency of the waves considered. VLF waves may travel up to $\lambda \sim 40^{\circ}$ before their reflection, while hiss waves are considered up to $\lambda \sim 30^{\circ}$ only. Here, the relevant calculations are performed for a constant mean amplitude of the waves, corresponding to the average spectral intensities measured by CRRES between $\lambda=5^{\circ}$ and $35^{\circ}$ (Meredith et al., 2007). Actually, the mean $B_{\mathrm{w}}^{2}$ was found to vary weakly with $\lambda$ for $\lambda<20^{\circ}$ in Cluster observations at $L=2-2.5$. However, we make use of the function $g(\theta)$ derived from fitting Cluster data at 0.89 to $3.5 \mathrm{kHz}$ (alternatively, we treat also the case of quasi-parallel waves).

To model the $g(X)$ distribution, we approximate Cluster statistics (see Agapitov et al., 2012a) near $1 \mathrm{kHz}$ by a polynomial dependence on latitude:

$$
\begin{aligned}
& X_{\mathrm{m}}=0.22-0.012 l+0.5 l^{2}-0.266 l^{3}+0.054 l^{4} \\
& X_{\mathrm{w}}=\sqrt{2}\left(0.19-0.19 l+0.51 l^{2}-0.24 l^{3}+0.044 l^{4}\right) .
\end{aligned}
$$

The same approximations of $X_{\mathrm{m}, \mathrm{w}}(\lambda)$ have been checked to be valid for waves in the range $0.9-3.6 \mathrm{kHz}$ at $L \in[2,2.5]$. A comparison of these approximations with the actual values of $X_{\mathrm{m}, \mathrm{w}}(\lambda)$ obtained from Cluster observations is shown in Fig. 7.

Table 1 gives a quick overview of the different wavenormal angle distributions that will be used for the different wave modes in the full numerical calculations. 


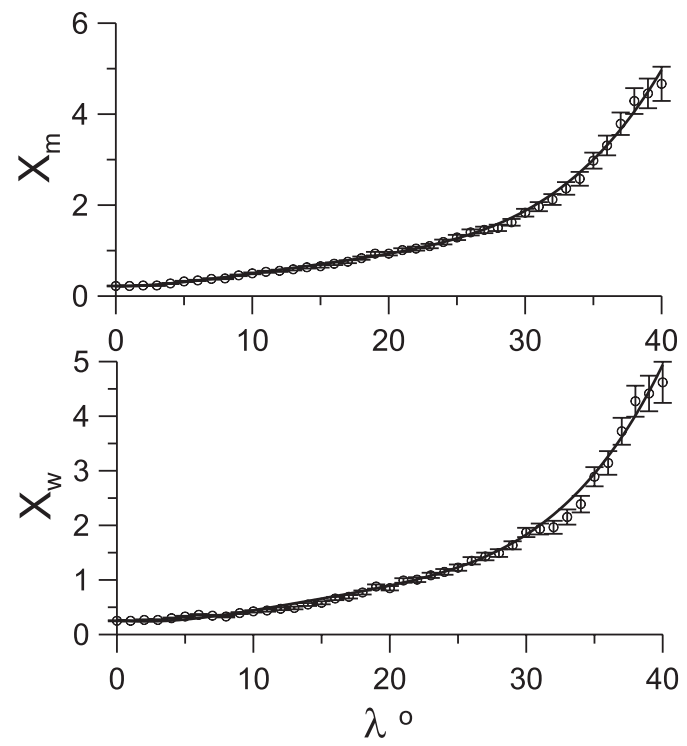

Fig. 7. Mean value $X_{\mathrm{m}}$ and variance $X_{\mathrm{w}}$ from Eq. (18) are shown by black color, while spacecraft observations are shown by circles.

\section{Parametric comparisons with full numerical simula- tions}

\subsection{Electron diffusion coefficients for interaction with lower-band chorus waves in the outer belt}

In this section we present pitch-angle diffusion coefficients $\langle D\rangle_{\mathrm{B}}$ averaged over the bounce oscillations of resonant electrons in a dipolar magnetic field (see details of the calculation scheme in Glauert and Horne, 2005; Artemyev et al., 2012b). We consider oblique lower-band chorus waves $\left(\omega_{\mathrm{m}} / \Omega_{\mathrm{c} 0}=\right.$ $0.35)$ dominant in the outer belt for $L \in$ [3.5-5.5]. For these calculations, we use the function $g(X)$ given in Eq. (17) and displayed in Fig. 6. For comparison, we also calculate $\langle D\rangle_{\mathrm{B}}$ for nearly parallel waves $\left(X_{\mathrm{m}}=0\right)$ with $X_{\mathrm{w}}=0.577$, which should be somewhat closer to the actual wave-normal angle distribution of chorus at large $L>6$ (these parameters were also used by Glauert and Horne, 2005; Horne et al., 2005). We use here a constant mean amplitude $B_{\mathrm{w}}=100 \mathrm{pT}$ corresponding to dayside active conditions $\mathrm{Kp} \sim 3$ (Shprits et al., 2007), and the equatorial magnetic field is calculated for $L=4.5$. Averaging over latitude is performed over the region $|\lambda|<\lambda^{+}=40^{\circ}$. For the main calculations, the full Appleton-Hartree dispersion in Eq. (17) is used. However, we also perform calculations of $\langle D\rangle_{\mathrm{B}}$ with the simplified dispersion relation $\omega=\Omega_{\mathrm{c}} \cos \theta /\left(1+\left(\Omega_{\mathrm{pe}} / k c\right)^{2}\right)$ to investigate the role of the ratio $\Omega_{\mathrm{pe}} / \Omega_{\mathrm{c} 0}$ (these results are marked as simplified DR, dispersion relation). Additionally, we show $\langle D\rangle_{\mathrm{B}}$ calculated with a hybrid scheme where the AppletonHartree dispersion is used everywhere (i.e., for the calculation of $\partial \omega / \partial k_{\|}$and $\partial \omega / \partial k$ for the normalization of $\left.g(X)\right)$, but the resonant roots $\left(\omega_{i, n}, k_{i, n}\right)$ are found from the simplified dispersion relation (these results are marked as simpli- fied $\left.\mathrm{DR}^{*}\right)$. Although the variation of the plasma frequency $\Omega_{\mathrm{pe}}$ along field lines can be taken into account (see, e.g., Summers and $\mathrm{Ni}, 2008$ ), the present analysis is restricted to the simpler case of a constant $\Omega_{\text {pe }}$ (believed to remain roughly valid up to nearly $\lambda^{+}=40^{\circ}$ ).

Figure 8 shows $\langle D\rangle_{\mathrm{B}}$ for given harmonic numbers $n$ calculated for three energies and four values of the ratio $\Omega_{\mathrm{pe}} / \Omega_{\mathrm{c} 0}$. Here, we perform a sum over $|n| \leq 60$ harmonics at low energy $E \leq 1 \mathrm{MeV}$ where the analytical estimate of the number of contributing (negative or positive) resonances $2 N_{\mathrm{r}}<$ 60 (see Sect. 3.1). At high energy $E=5 \mathrm{MeV}$, however, the number of contributing resonances $2 N_{\mathrm{r}}$ reaches 160 at $\Omega_{\mathrm{pe}} / \Omega_{\mathrm{c} 0}=10$; then a sum over $|n| \leq 160$ harmonics is performed. Typically, $\langle D\rangle_{\mathrm{B}}$ increases strongly at small equatorial pitch angles $\alpha_{0}$ for oblique waves as compared to nearly parallel ones (compare black and grey solid curves). This increase is produced by the growth of almost all the individual rates in the vicinity of the loss cone, while only the first resonance is available for parallel waves alone (Shklyar and Matsumoto, 2009; Mourenas et al., 2012b). For large values of $\Omega_{\mathrm{pe}} / \Omega_{\mathrm{c} 0}>2$, we also show $\langle D\rangle_{\mathrm{B}}$ calculated with the simplified DR and simplified DR* (black dashed and thin solid curves). One can see that $\langle D\rangle_{\mathrm{B}}$ calculated with the simplified $\mathrm{DR}^{*}$ almost coincides with $\langle D\rangle_{\mathrm{B}}$ obtained with the Appleton-Hartree dispersion for $\alpha_{0}>15^{\circ}$. Electrons with $\alpha_{0}<10^{\circ}$ can reach high latitudes, since their mirror points are located at $|\lambda|>50^{\circ}$. However, the latitude upper bound in our calculations is taken at $|\lambda|=\lambda^{+}=40^{\circ}$, where $\Omega_{\mathrm{c} 0} / \Omega_{\mathrm{c}} \approx 1 / 7$ and $\left(\Omega_{\mathrm{pe}} / \Omega_{\mathrm{c}}\right)^{2} \approx(1 / 50)\left(\Omega_{\mathrm{pe}} / \Omega_{\mathrm{c} 0}\right)^{2}$. This gives $\left(\Omega_{\mathrm{pe}} / \Omega_{\mathrm{c}}\right)^{2}<2$ for $\Omega_{\mathrm{pe}} / \Omega_{\mathrm{c} 0}<10$. Only there, in the close vicinity of the loss cone, can a substantial difference appear between the two calculations of $\langle D\rangle_{\mathrm{B}}$ due to corrections to resonant roots $\left(\omega_{i, n}, k_{i, n}\right)$ induced by the modification of the dispersion relation. As a result, the latitude of resonance at peak wave power decreases (see Fig. 4) and diffusion decreases too with the simplified dispersion, because $\Delta_{\mathrm{n}} G_{\theta}$ in Eq. (1) increases with latitude (Mourenas et al., 2012a). Moreover, in the presence of an oblique wave distribution $g(X)$ with a large variance $X_{\mathrm{w}}$, the normalization of $g(X)$ in Eq. (2) can also modify $\langle D\rangle_{\mathrm{B}}$ by up to a factor of 2 when using the simplified dispersion relation at medium to large pitch angles $30^{\circ}<\alpha_{0}<75^{\circ}$. In agreement with Glauert and Horne (2005), there is no substantial difference between $\langle D\rangle_{\mathrm{B}}$ calculated with the Appleton-Hartree dispersion and with the simplified DR in the case of nearly parallel waves at $E \geq 100 \mathrm{keV}$ (not shown here).

The analytical estimate in Eq. (6) of $\langle D\rangle_{\mathrm{B}}$ for oblique waves agrees fairly well with the full numerical solutions in Fig. 8 in the range $\Omega_{\mathrm{pe}} / \Omega_{\mathrm{c} 0}>4$ and $\alpha_{0}>10^{\circ}$ where the simplified roots are approximately correct. In particular, it is worth noting that the $1 / \sin \alpha_{0}$ decrease of analytical diffusion rates with pitch angle coincides with the actual drop-off of the numerical diffusion rates. As explained in Sect. 3, although the discrepancy between analytical and numerical results near the loss-cone edge may be important, it will prove 

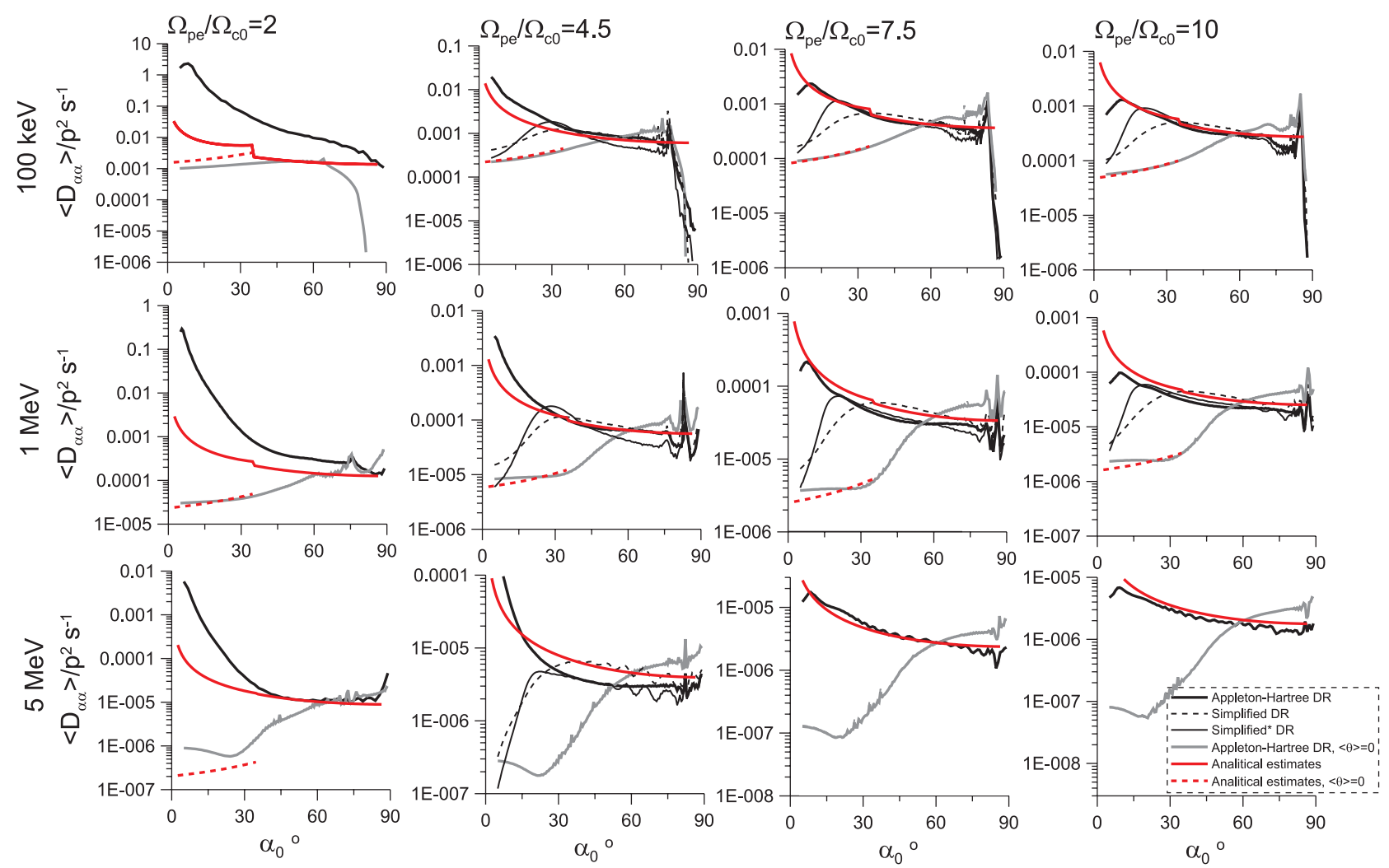

Fig. 8. $\left\langle D_{\alpha \alpha}\right\rangle$ for three values of electron energy and four values of the ratio $\Omega_{\mathrm{pe}} / \Omega_{\mathrm{c} 0}$, for quasi-parallel and oblique chorus waves. Full numerical solutions as well as the corresponding analytical estimates in Eqs. (4) and (6) are displayed.

negligible in lifetime calculations where $1 /\left(\tan \alpha_{0}\langle D\rangle_{\mathrm{B}}\right)$ is integrated over the whole pitch-angle domain (see Eq. 7). In the lowest density case $\Omega_{\mathrm{pe}} / \Omega_{\mathrm{c} 0}=2$, analytical estimates still roughly match the full numerical rates for $\alpha_{0}>30^{\circ}$ and $E \geq 1 \mathrm{MeV}$. Numerical rates $\langle D\rangle_{\mathrm{B}}$ are then much larger than the estimates at lower pitch angles, which should not lead to large errors in analytical lifetimes. At the lowest density and for $E=100 \mathrm{keV}$, however, the analytical estimate in Eq. (6) significantly underestimates diffusion at $\alpha_{0}<60^{\circ}$, because the corresponding estimated number of contributing resonances $N_{\mathrm{r}} \sim 0.9$ is very small with the simplified dispersion, while many more harmonics actually contribute with the full dispersion (see Fig. 8). It shows that the analytical estimate in Eq. (6) is valid at least between $100 \mathrm{keV}$ and $5 \mathrm{MeV}$ for $\Omega_{\mathrm{pe}} / \Omega_{\mathrm{c} 0}>4$, while an additional condition $N_{\mathrm{r}}>2$ is required for $\Omega_{\mathrm{pe}} / \Omega_{\mathrm{c} 0}<4$, leading to a global validity limit given approximately by $E(\mathrm{MeV})>\max \left(0.1, \Omega_{\mathrm{c} 0}^{3} /\left(\Omega_{\mathrm{pe}}^{2} \omega_{\mathrm{m}}\right)\right)$. Finally, in the case of a quasi-parallel wave distribution $(\theta<$ $\left.45^{\circ}\right)$, the analytical estimate in Eq. (4) of $\langle D\rangle_{\mathrm{B}}$ near the loss cone can be seen in Fig. 8 to correspond rather well to the full numerical solution at all densities $\Omega_{\mathrm{pe}} / \Omega_{\mathrm{c} 0}>2$ and energies $E=100 \mathrm{keV}$ to $2 \mathrm{MeV}$. It is only plotted for $\alpha_{0}<40^{\circ}$, because the analytical $1 / \cos ^{2} \alpha_{0}$ variation in Eq. (4) is not expected to hold at larger pitch angles (Mourenas and Ripoll, 2012). Nevertheless, it is this low- $\alpha_{0}$, low- $\left\langle D_{\alpha \alpha}\right\rangle$ range that actually determines the lifetime value in this case (Albert and Shprits, 2009; Mourenas and Ripoll, 2012).

For $E=5 \mathrm{MeV}$ and at high density, the analytical estimates of the small- $\theta$ cyclotron diffusion rates are null, simply because the analytical estimate in Eq. (5) of $\lambda_{R}$ is then such that, analytically, resonance occurs only above the latitude upper bound $\lambda^{+}=40^{\circ}$, where wave intensity was assumed to vanish. The latitudinal range of resonance is very narrow, and the mean latitude of resonance is then very slightly overestimated by the approximate Eq. (5), leading to an underestimation of the cyclotron diffusion rates at $5 \mathrm{MeV}$. Without such an upper bound $\lambda<\lambda^{+}$, however, we have checked that the analytical estimate in Eq. (4) of $\langle D\rangle_{\mathrm{B}}$ remains accurate even at high energy. Anyway, this discrepancy occurs only for very low diffusion rates, corresponding to very large lifetimes (see below). It is therefore not really important for most practical applications such as lifetime estimates in monthslong numerical simulations of the radiation belts.

To explain the difference between the results obtained for oblique waves (with $g(X)$ from Fig. 6) and for nearly parallel waves $\left(X_{\mathrm{m}}=0, X<1\right)$, we plot the function $\hat{G}(\theta, \lambda)=$ $\sum_{\omega} G_{\omega} G_{\theta}$ for particular values of particle energy, equatorial pitch angle and harmonic number (see Fig. 9). Note that diffusion rates are proportional to $\sim \int \hat{G}(\theta, \lambda) d \lambda d \theta$. For nearly parallel waves, the maximum of $\hat{G}$ corresponds to 


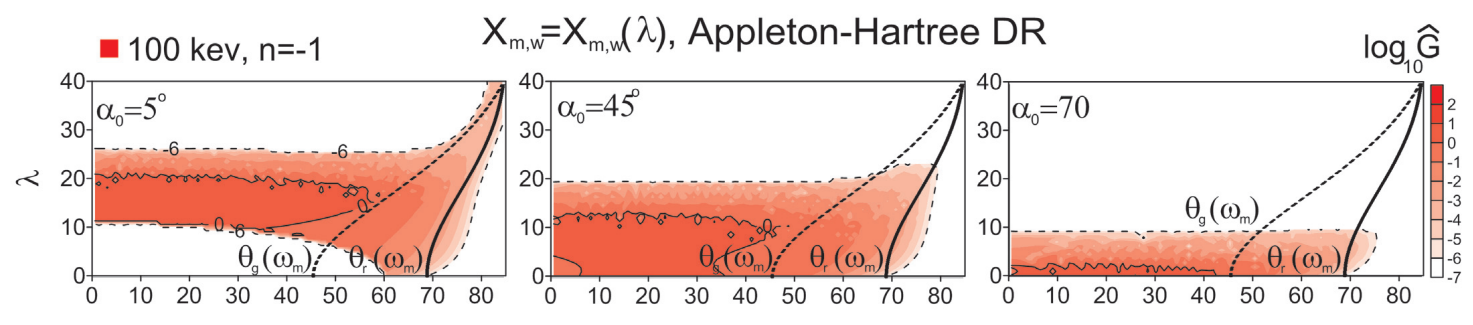

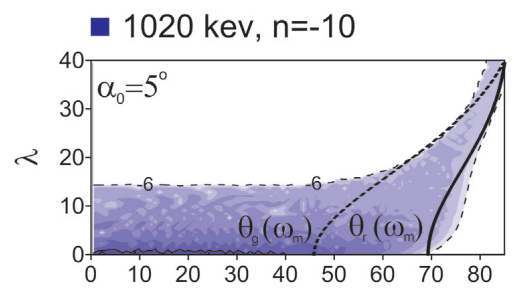

$\theta$

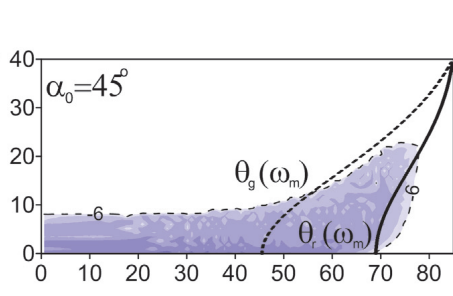

$\theta$

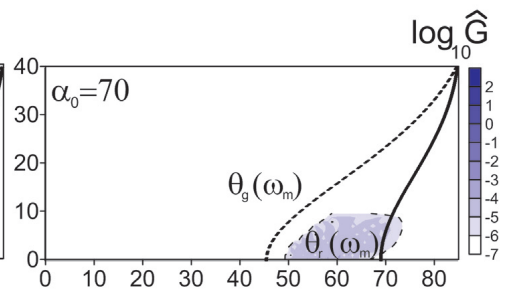

$\theta$

Fig. 9. Function $\hat{G}(\theta, \lambda)$ for two $n$ and particle energies (see text for details). Here $\Omega_{\mathrm{pe}} / \Omega_{\mathrm{c} 0}=4.5$.

$\theta<40^{\circ}$ for all energies, pitch angles, and harmonics. This $\theta$ range corresponds roughly to $\alpha_{0}>60^{\circ}$ (since the maximum of Bessel functions occurs for $\alpha \approx \pi / 2-\theta$; see Mourenas and Ripoll, 2012, and explanations above). It implies that pitch-angle diffusion by parallel waves will be important mainly for $\alpha_{0}>60^{\circ}$, as seen in Fig. 8. For oblique waves, conversely, the maximum value of $\hat{G}$ depends on $\lambda$ due to $X_{\mathrm{m}, \mathrm{w}}=X_{\mathrm{m}, \mathrm{w}}(\lambda)$. Then, the function $\hat{G}$ has finite values up to at least $\theta=80^{\circ}$ and varies weakly with $\theta$ (see $n=-1$ harmonic for $100 \mathrm{keV}$ particles and $n=-10$ harmonic for $1 \mathrm{MeV}$ particles). In particular, $\hat{G}$ attains now finite values in the $\theta$ domain comprised between the Gendrin and resonance cone angles, falling sharply when $\theta$ gets close to $\theta_{\mathrm{r}}(\omega)$ as explained by Mourenas et al. (2012b) and Albert (2012). As a result, all particles with $\alpha_{0}>20^{\circ}$ may be scattered with approximately the same rate (one can find a peak of $\langle D\rangle_{\mathrm{B}}$ at intermediate values of $\alpha_{0}$ in Fig. 8). At high latitudes, cyclotron resonance becomes available only between the Gendrin and resonance cone angles. The increase of $\langle D\rangle_{\mathrm{B}}$ for $\alpha_{0}<10^{\circ}$ corresponds to the sum of factors $\Delta_{\mathrm{n}}$ over $4 N_{\mathrm{r}}$ harmonics. Note that the nonzero value of $\hat{G}(\theta, \lambda)$ for $\theta>\theta_{\mathrm{r}}\left(\omega_{\mathrm{m}}\right)$ is explained by the finite range $\Delta \omega$ of $\omega$ variation in the calculation of $\hat{G}(\theta, \lambda)$; i.e., $\omega<\omega_{\mathrm{m}}+\Delta \omega$ while $\theta_{\mathrm{r}}$ is calculated for $\omega=\omega_{\mathrm{m}}$.

To better understand the behavior of $\langle D\rangle_{\mathrm{B}}$, it is useful to plot the local $D_{\alpha \alpha}(\theta, \lambda)$ diffusion coefficient for given values of $\alpha_{0}$ and $E\left(\langle D\rangle_{\mathrm{B}}=\int D_{\alpha \alpha} d X d \lambda\right)$. Figures 10 and 11 show these local pitch-angle diffusion coefficients for $\Omega_{\mathrm{pe}} / \Omega_{\mathrm{c} 0}=4.5$ and 7.5 , respectively, calculated with the Appleton-Hartree DR and with the simplified DR* The left-hand panels correspond to small equatorial pitch angle $\alpha_{0}=5^{\circ}$ and the right-hand panels to $\alpha_{0}=45^{\circ}$. From top to bottom, one considers $E=100 \mathrm{keV}$ electrons for $n=-1$ and -5 , then $E=1 \mathrm{MeV}$ electrons for $n=-1,-5$, and -10 in both figures. The average values of $\theta_{\mathrm{g}}(\lambda)$ and $\theta_{\mathrm{r}}(\lambda)$ calcu- lated at the frequency $\omega=\omega_{\mathrm{m}}$ of peak wave power are also indicated for convenience (solid black and dotted black lines, respectively). It has been established theoretically (Mourenas and Ripoll, 2012; Mourenas et al., 2012b) that the contributing resonances for quasi-parallel waves alone are such that $1 \leq|n| \leq N_{\max }^{s}$ (see also Sect. 3.1). For $\Omega_{\mathrm{pe}} / \Omega_{\mathrm{c} 0}=4.5-$ 7.5 , one finds $N_{\max }^{s} \sim 1.5-2.3$ and $5-8$ for $E=100 \mathrm{keV}$ and $1 \mathrm{MeV}$, respectively. It is plain to see in Figs. 10 and 11 that, for low-order resonances such that $1 \leq|n| \leq N_{\max }^{s}$, the most important contribution to diffusion indeed comes from the range $\theta<45^{\circ}$, while, for higher-order resonances such that $|n|>N_{\max }^{s}$, diffusion is significant only for $\theta_{\mathrm{g}} \leq \theta<\theta_{\mathrm{r}}$ as expected from other analytical considerations (Mourenas et al., 2012b). The proposed approximation (Mourenas et al., $2012 b$ ) of the wave-normal angle distribution by two distributions at $\theta<45^{\circ}$ and $\theta>\max \left(45^{\circ}, \theta_{\mathrm{g}}\right)$ appears therefore vindicated. Oscillations in the magnitude of $D$ can be seen in this large- $\theta$ range for fixed $n$ and increasing latitude $\lambda$ or wave-normal angle $\theta$. These oscillations are due to modulations of the Bessel functions $J_{\mathrm{n}}(x)$ with $x \sim n \tan \theta \tan \alpha$ as the resonant pitch-angle value $\alpha$ grows with $\lambda$ up to the mirror point (where the latitude of mirror points is $\lambda_{b} \sim$ $\sqrt{2} /\left(3 \tan \alpha_{0}\right)$ from adiabatic invariance (Mourenas et al., 2012b)) or $\lambda^{+}$, or as $\theta$ increases up to $\theta_{\mathrm{r}}$ at a given latitude. It is also worth noting in Figs. 10 and 11 the close agreement between the local $D$ calculated with the Appleton-Hartree DR and with the simplified $\mathrm{DR}^{*}$ for $\alpha_{0}=45^{\circ}$, demonstrating that the approximate roots are correct in the medium to large pitch-angle range. As expected, the agreement between the full DR $D$ and the simplified one becomes better at higher density for both values of $\alpha_{0}$.

Now, let us focus on the large- $\theta$ domain. Significantly contributing resonances are then expected to be such that $N_{r} / 3 \leq|n| \leq 3 N_{\mathrm{r}}$, with $D(n)$ decreasing roughly like $1 / n^{2}$ for $|n| \gg N_{\mathrm{r}}$ (Mourenas et al., 2012b). For the cases in 


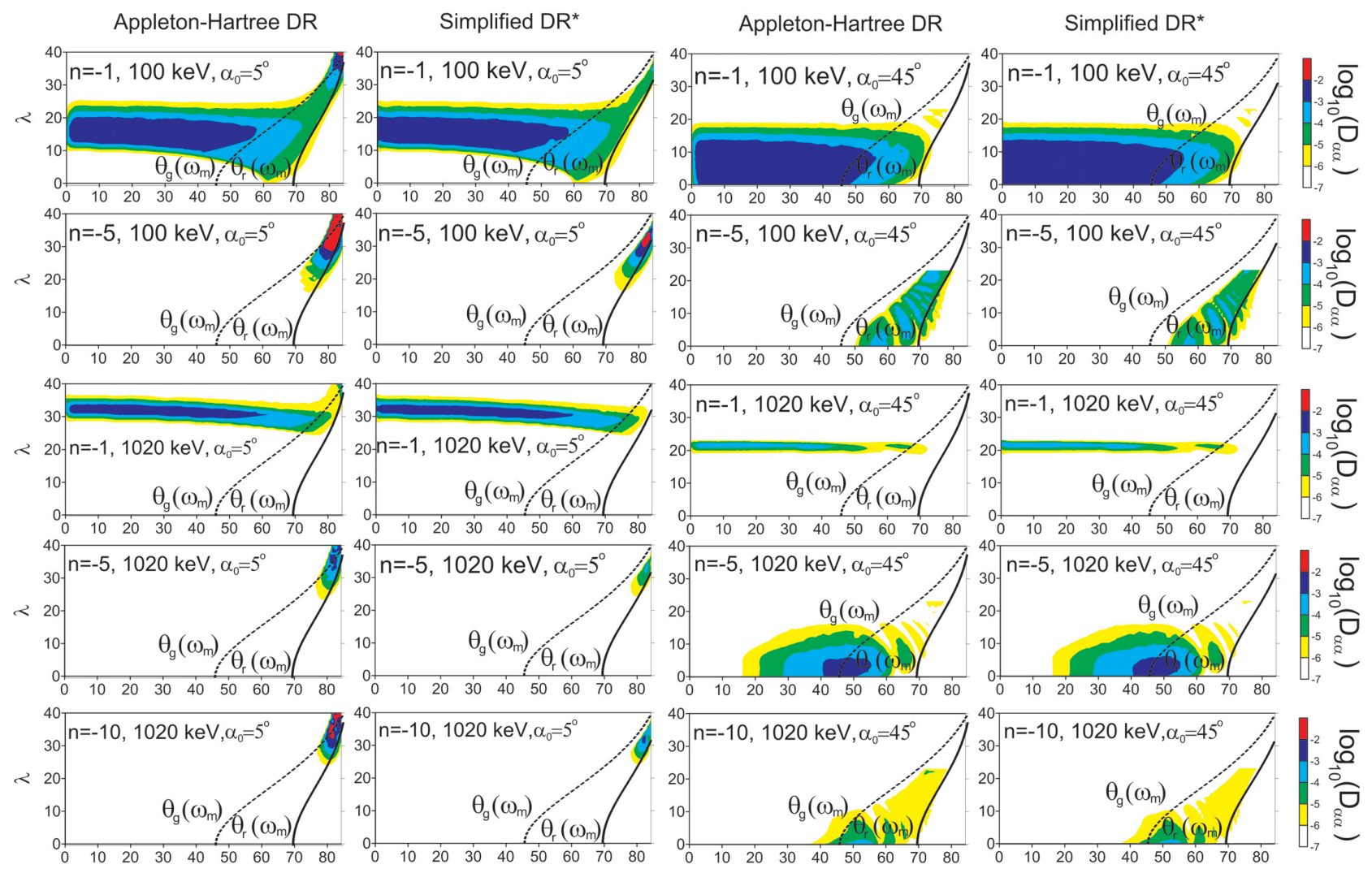

Fig. 10. Local diffusion coefficient $D_{\alpha \alpha}$ for various $n$, particle energies and equatorial pitch angles (see text for details). The black solid curve shows the position of resonance cone angle $\theta_{\mathrm{r}}$. Black dashed curves show the position of the Gendrin angle $\theta_{\mathrm{g}}$. Here $\Omega_{\mathrm{pe}} / \Omega_{\mathrm{c} 0}=4.5$.

Figs. 10 and 11, one finds $N_{\mathrm{r}} \sim 2$ to 3 for $E=100 \mathrm{keV}$, while for $E=1 \mathrm{MeV}$ one gets for $\Omega_{\mathrm{pe}} / \Omega_{\mathrm{c} 0}=4.5$ (7.5) that $N_{\mathrm{r}} \sim 6(10)$ at $\alpha_{0}=45^{\circ}$ and $N_{\mathrm{r}} \sim 9(15)$ at $\alpha_{0}=5^{\circ}$. Larger values of $D$ are found for $|n| \sim N_{\mathrm{r}}$ as predicted, for example in Fig. 10 at $1 \mathrm{MeV}$ for $n \sim-5$ at $\alpha_{0}=45^{\circ}$ and for $n \sim-10$ at $\alpha_{0}=5^{\circ}$. At higher $|n|$, a clear decrease can also be seen for $n=-10 \sim-1.7 N_{\mathrm{r}}$ as compared to $n=-5$ for $\alpha_{0}=45^{\circ}$ in the same figure. In Figs. 10 and 11, $D$ goes down to zero for $n=-1$ at $1 \mathrm{MeV}$, which corresponds to the range $|n|<N_{\mathrm{r}} / 3$. For $|n|<N_{\mathrm{r}}$, resonance occurs farther and farther away from the equator (Mourenas et al., 2012b). The upper latitude of diffusion obtained in both figures corresponds to the estimate $\lambda_{\mathrm{b}}$ for $\alpha_{0}>20^{\circ}$, reaching $\lambda^{+}=40^{\circ}$ at smaller pitch angles. For $\alpha_{0}=5^{\circ}$ (a value typical of the range $\alpha_{0}<$ $\left.10^{\circ}\right), D$ attains significant values only at high latitudes such that $\lambda>\left(2\left(\omega_{\mathrm{m}} /\left[\Omega_{\mathrm{c} 0} \sin \alpha_{0}\right]\right)^{2 / 27}-2\right)^{1 / 2}$ (Mourenas et al., 2012a). With the roots from the simplified dispersion relation, the maximum latitude where $D$ is important agrees well with the analytical estimate $\lambda_{\mathrm{MM}} \sim 35^{\circ}$ (Mourenas et al., 2012a). With the full Appleton-Hartree DR, however, $D$ remains high up to $\lambda^{+}=40^{\circ}$. With the simplified $\mathrm{DR}^{*}$, resonance in the vicinity of the loss cone is therefore shifted to lower latitudes, and this shift results in a decrease of diffu- sion. However, this difference between the two DRs is only important near the loss cone at the highest latitudes.

The above-discussed variation of individual bounceaveraged diffusion coefficients $\left\langle D^{n}\right\rangle_{\mathrm{B}}$ as a function of $n$ for quasi-parallel or oblique chorus waves is emphasized in Fig. 12. The main result in Fig. 12 is the very large increase of the number of contributing resonances with oblique whistler waves $\left(|n| \sim 2 N_{\mathrm{r}}\right)$ as compared to nearly parallel waves $\left(|n|<N_{\text {Max }}^{s}<N_{\mathrm{r}}\right)$. It explains the higher diffusion rates presented in Fig. 8 for oblique waves. In general, diffusion by nearly parallel waves occurs only for $|n| \leq N_{\mathrm{Max}}^{s}$, while diffusion by oblique waves occurs principally for $N_{\mathrm{r}} / 3 \leq|n| \leq$ $2 N_{\mathrm{r}}$, with a progressive but steep decrease above $N_{\mathrm{r}}$ as well as below $N_{\mathrm{r}} / 3$ as expected (Mourenas et al., 2012b). Near the loss-cone edge (for $\alpha_{0}<10^{\circ}$ ), however, more intense highorder resonances become available for diffusion by oblique waves when the density decreases with the full AppletonHartree DR than from the simple estimate $<2 N_{\mathrm{r}}$ obtained with the simplified DR. At such small equatorial pitch angles $\alpha_{0}<\pi / 2-\Delta \theta$, only the first cyclotron resonance contributes in the case of quasi-parallel waves (see the grey curve in the left panels of Fig. 12), while at larger pitch angles, all the $|n|<N_{\text {Max }}^{s}$ resonances can then be important (see right panels in Fig. 12 and discussion in Mourenas and Ripoll, 2012). 


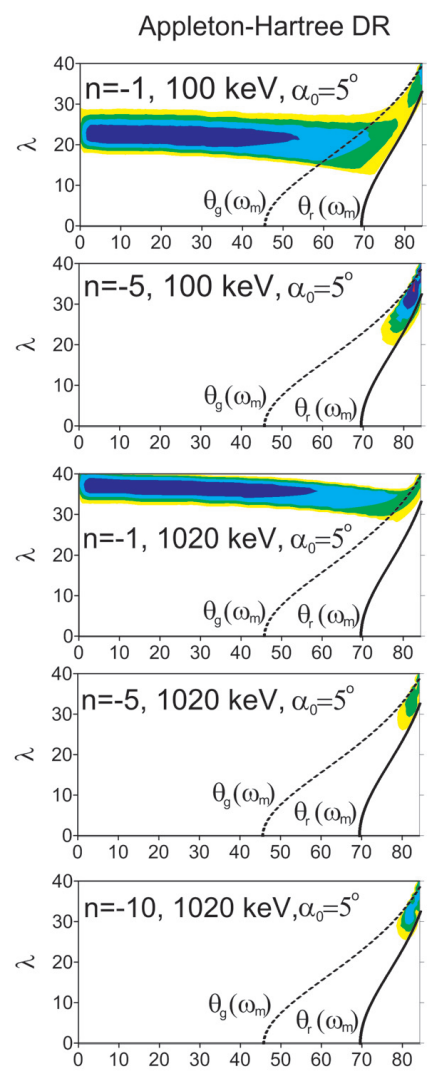

$\theta$
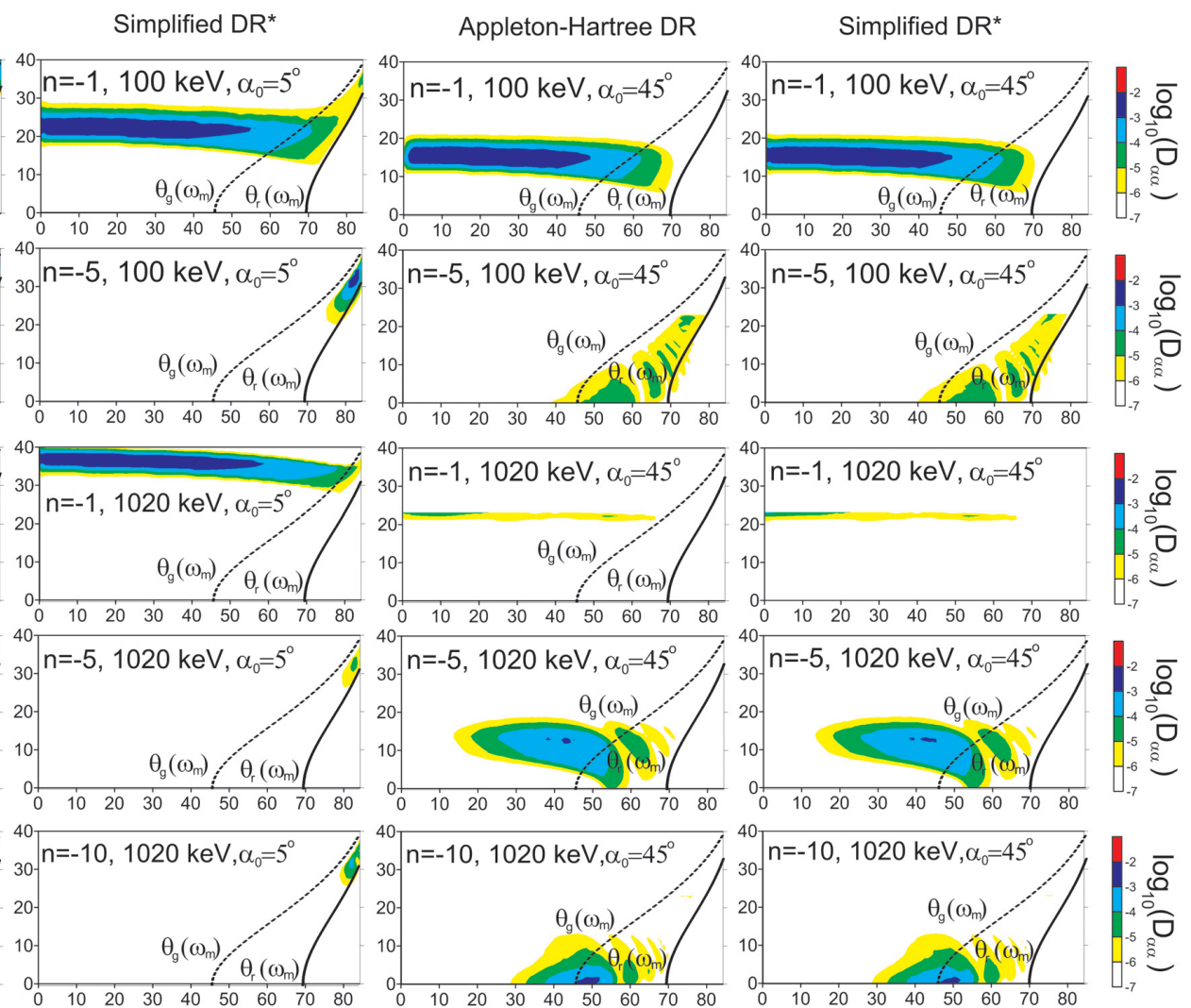

Fig. 11. Local diffusion coefficient $D_{\alpha \alpha}$ for various $n$, particle energies and equatorial pitch angles (see text for details). The black solid curve shows the position of resonance cone angle $\theta_{\mathrm{r}}$. Black dashed curves show the position of the Gendrin angle $\theta_{\mathrm{g}}$. Here $\Omega_{\mathrm{pe}} / \Omega_{\mathrm{c} 0}=7.5$.

\subsection{Electron lifetimes in the outer belt and scaling laws}

The analytical lifetime estimates provided in Eq. (9) for quasi-parallel waves and in Eq. (12) for oblique waves are compared with the numerically calculated electron lifetimes in Figs. 13, 14 over a broad parameter range representative of the outer belt (similar to Fig. 8). Equation (9) for quasiparallel waves reproduces rather accurately the full numerical lifetimes over the whole parameter range. The discrepancy is most often smaller than a factor of 1.5. At the highest energies (and at higher energy for smaller density), a sudden increase of the lifetimes can be noticed: it is due to the reduction of the latitudinal range of cyclotron resonance, as resonance occurs at higher and higher latitude corresponding to $\Omega_{\mathrm{c}}(\lambda) / \Omega_{\mathrm{c} 0} \approx\left(p \varepsilon_{\mathrm{m} 0}\right)^{2 / 3}$ (Mourenas and Ripoll, 2012; Mourenas et al., 2012b). When cyclotron resonance is not anymore available below the assumed latitude upper bound $\lambda^{+}=40^{\circ}$ of wave presence, only Landau resonance remains and the corresponding lifetimes are much larger (Horne et al., 2005). The energy threshold for this steep increase of lifetime is rather accurately predicted by the analytical second-order estimate in Eq. (5) of the latitude of resonance (within $15 \%$ of the actual value). From Eqs. (4)-(5), the increase of life- times should occur at a nearly constant value of $p \varepsilon_{\mathrm{m} 0}$, i.e., at energies such that $p \propto \Omega_{\mathrm{c} 0} / \Omega_{\mathrm{pe}}$ for fixed chorus frequency, which is exactly what is observed in Fig. 13. This situation corresponds to very large lifetimes near or above 100 days for high-amplitude $100 \mathrm{pT}$ waves (lifetimes would be even larger for smaller amplitudes). Moreover, we have checked that if we remove this assumed upper latitude cutoff at $\lambda^{+}$, analytical lifetimes remain correct even at higher energies.

As concerns oblique waves, the analytical lifetime estimate in Eq. (12) is also found to be in good agreement with the full numerical calculations over most of the considered parameter domain, i.e., for $E(\mathrm{MeV})>$ $\max \left(0.1, \Omega_{\mathrm{c} 0}^{3} /\left(\Omega_{\mathrm{pe}}^{2} \omega_{\mathrm{m}}\right)\right)$. This corresponds to the whole density (or $L$ shell) outer-belt domain for $E \geq 1 \mathrm{MeV}$ and to $\Omega_{\mathrm{pe}} / \Omega_{\mathrm{c} 0}>4$ for $E>100 \mathrm{keV}$. In this domain, the discrepancy between analytical estimates and the numerical lifetimes remains smaller than a factor of 2 . This is much smaller than the uncertainties associated with outer-belt density models (Sheeley et al., 2001; Denton et al., 2006; Ozhogin et al., 2012) and chorus intensity models (Meredith et al., 2001; Shprits et al., 2007; Li et al., 2011; Artemyev et al., 2012b). Therefore, the proposed analytical lifetimes could prove useful for performing extensive numerical simulations of the 
radiation belts or to investigate in detail the sensibility of global belt simulations to various parameters. As noted before, the simplified dispersion used in the analytical calculations is more accurate at higher density. The agreement of analytical lifetimes with full numerical results is thus expected to improve steadily as $\Omega_{\mathrm{pe}} / \Omega_{\mathrm{c} 0}$ increases. It is exactly the behavior that is observed in Figs. 13 and 14. In the latter figure, lifetimes are seen to vary asymptotically like $\tau_{\mathrm{s}} \propto \Omega_{\mathrm{pe}}^{14 / 9}$ and $\tau_{s+l} \propto \Omega_{\mathrm{pe}}$ at high density, as predicted in Eqs. (14)(15). Finally, Fig. 15 shows that lifetimes (both analytical and numerical) are weakly dependent on the mean lowerband chorus frequency for typical values $\omega_{\mathrm{m}} / \Omega_{\mathrm{c} 0}=0.15$ to 0.4 (Horne et al., 2005; Shprits et al., 2007), especially at high energy. For $\omega_{\mathrm{m}} / \Omega_{\mathrm{c} 0}>0.45$, a significant portion of the wave power lies inside the upper-band chorus range, not covered by the present analytical estimates. Actually, the approximation $\gamma \omega / \Omega_{\mathrm{c}} \ll|n|$ used to simplify the argument of the Bessel functions in Eq. (1) becomes less reliable for $\omega=\omega_{\mathrm{m}}+\Delta \omega>0.5 \Omega_{\mathrm{c} 0}$. An application of our analytical estimates for upper-band chorus must therefore be ruled out. It is probably the explanation for the steeper increase of lifetimes in full numerical solutions than in the analytical estimates at $\omega_{\mathrm{m}} / \Omega_{\mathrm{c} 0}>0.45$ for oblique waves. Lifetimes are generally one order of magnitude smaller with very oblique waves included than with quasi-parallel waves alone. Such a strong reduction of timescales might actually contribute to explain the large and rapid trapped electron density dropouts observed in the outer radiation belt during high geomagnetic activity periods at $L=4$ to 5 (Tu et al., 2010; Kim et al., 2011).

\subsection{Role of very oblique waves}

Still, the preceding lifetimes were evaluated for constant wave amplitudes. For more realistic calculations, the wave power distribution $B_{\mathrm{w}}^{2}(\theta)$ as a function of $\theta$ should also be taken into account. It leads to a reduced amount of wave power at large wave-normal angles as compared to $\theta<45^{\circ}$ (see Fig. 2). Therefore, the effect of oblique waves in lifetimes should be reduced. The bounce-averaged diffusion coefficient calculated by multiplying the $\theta$ distribution $g(\theta)$ by a function $B_{\mathrm{w}}^{2}(\theta)$ is displayed in Fig. 16 for the same oblique wave distribution $g(\theta)$ as before and $E=1 \mathrm{MeV}$ (similar results are obtained for $E=0.1$ to $5 \mathrm{MeV}$, not shown here). The wave intensity $B_{\mathrm{w}}^{2}$ at $\lambda>20^{\circ}$ is divided (via $g(\theta)$ ) by factors $1.5,2,5,20$ and 100 for $\theta>60^{\circ}$ to demonstrate this effect. The corresponding reduction of pitch-angle diffusion occurs for $\alpha_{0}<\omega_{\mathrm{m}} / \Omega_{\mathrm{c} 0}$ (i.e., $\alpha_{0}<20^{\circ}$ ) only, as predicted in Sect. 3. Moreover, when oblique wave amplitudes at $\lambda>20^{\circ}$ are reduced by less than a factor of 2 , the diffusion rate is almost unaffected. Actual lifetimes at $L \sim 4$ to 5.5 can therefore be calculated without any reduction factor, like in Fig. 13, and they are well estimated by Eqs. (12)-(15). At larger $L>5.5$, they should be comprised between 30 and
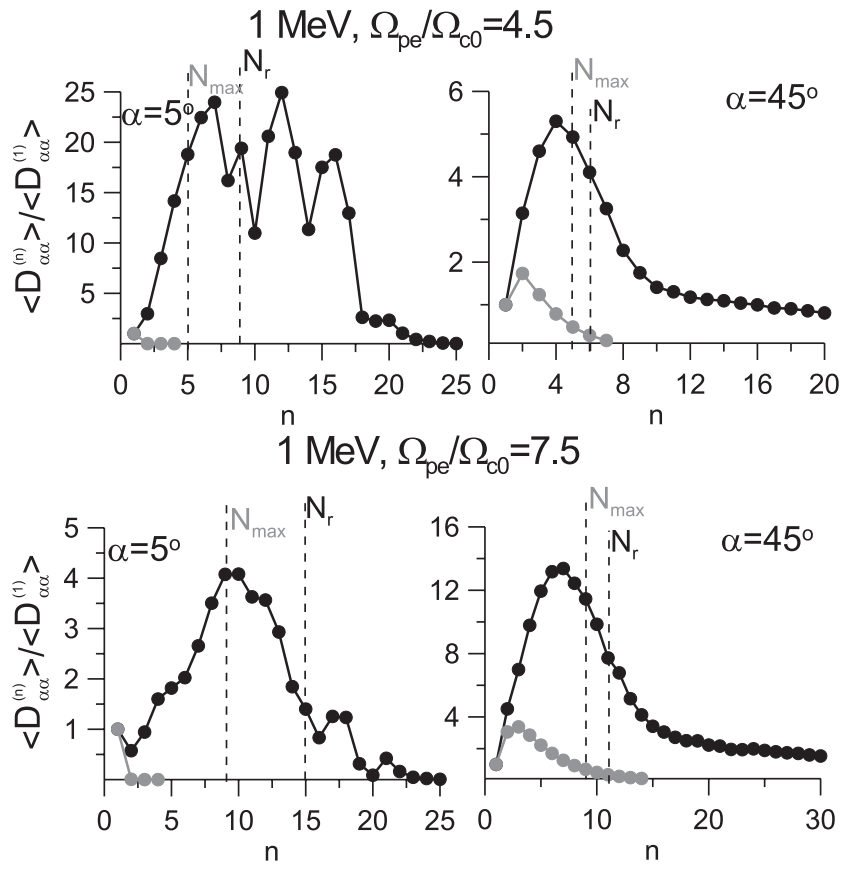

Fig. 12. Individual bounce-averaged diffusion coefficient $\left\langle D^{n}\right\rangle$ as a function of $n$. The black solid and black dashed vertical lines show the values of $N_{\text {Max }}^{s}$ and $N_{\mathrm{r}}$, respectively. Here $E=1 \mathrm{MeV}$ and $\alpha_{0}=$ $5^{\circ}, 45^{\circ}$.

$60 \%$ of the quasi-parallel-wave lifetime (Eqs. 9-14) (see the end of Sect. 3).

\subsection{Role of mean amplitude $B_{w}$ variation with $\lambda$}

The preceding comparisons have been achieved for a (mean) wave intensity independent of latitude. It is important to check if full numerical lifetimes obtained with a realistic latitude-varying wave intensity $B_{\mathrm{w}}^{2}(\lambda)$ (see Artemyev et al., $2012 \mathrm{~b}$ ) can also be recovered with the approximate analytical model by means of using only an average intensity. Figure 17 shows the full numerical $\langle D\rangle_{\mathrm{B}}$ calculated for $\mathrm{Kp}<3$ for different values of energy and plasma density. We use the same parameter range as before and a distribution $g(X)$, with $B_{\mathrm{w}}(\theta)$ kept fixed in order to demonstrate each effect separately. One can see that the approximation of a constant rms amplitude $B_{\mathrm{w}}=6 \mathrm{pT}$ (corresponding to Fig. 2) gives diffusion rates very similar to the diffusion rates calculated with a realistic distribution $B_{\mathrm{w}}^{2}(\lambda)$. However, due to a significant minimum in wave power $B_{\mathrm{w}}^{2}(\lambda)$ in the vicinity of the equator on the dayside in Cluster statistics (see, e.g., Artemyev et al., 2012b; Agapitov et al., 2012a), the realistic diffusion rate is significantly smaller than $\langle D\rangle_{\mathrm{B}}$ calculated with a fixed $B_{\mathrm{w}}$ at very large pitch angles $\alpha_{0}>80^{\circ}$, especially for low energy $E \sim 100 \mathrm{keV}$. The corresponding lifetimes are almost identical to numerical lifetimes evaluated with a constant $B_{\mathrm{w}}$ for medium to high energy electrons (as for $E=1 \mathrm{MeV}$ ), but sensibly increased at low energy $E \sim 100 \mathrm{keV}$ (by a factor 

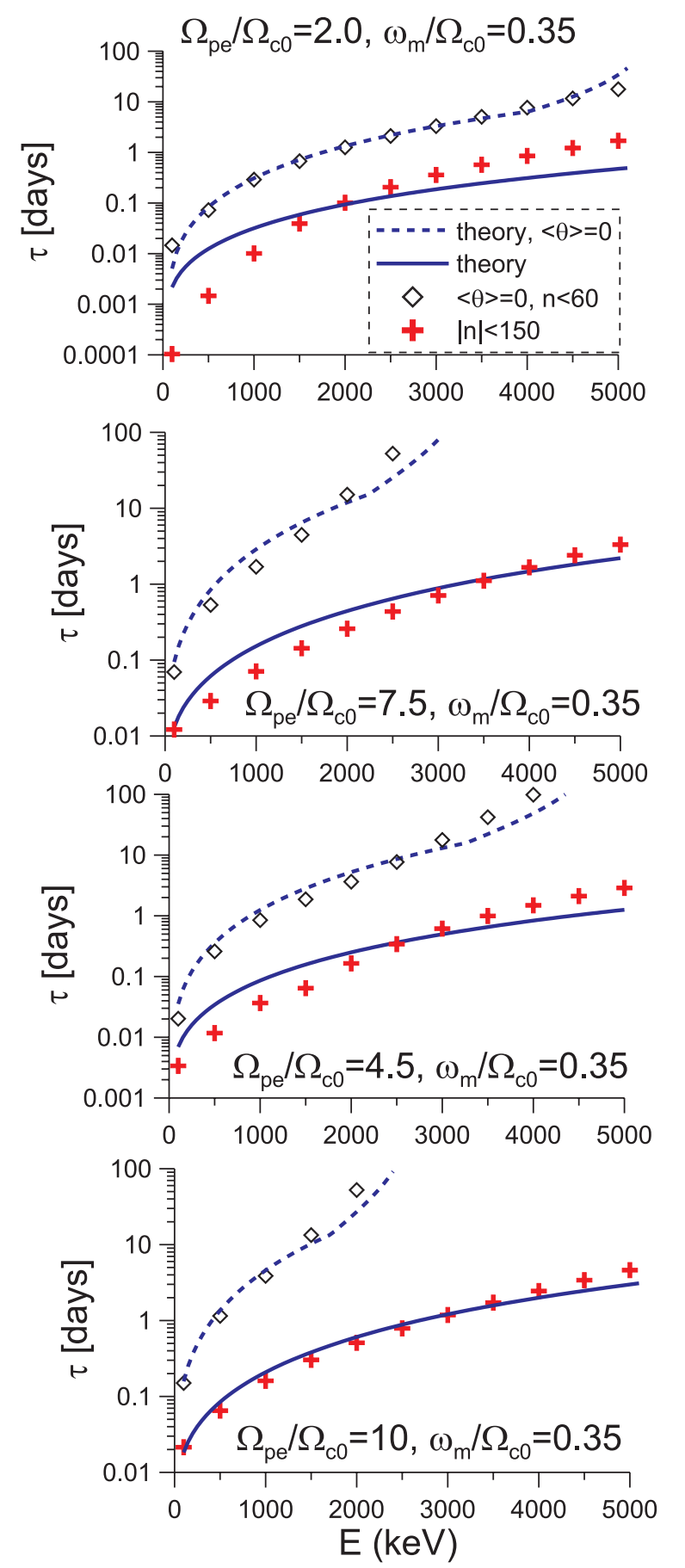

Fig. 13. Electron lifetimes for four values of the ratio $\Omega_{\mathrm{pe}} / \Omega_{\mathrm{c} 0}$, for quasi-parallel and oblique chorus waves. Full numerical solutions as well as the corresponding analytical estimates in Eqs. (9) and (12) are displayed.

of 5 to 10). Nonetheless, it is worth emphasizing that Cluster statistics are probably slightly biased near the equator due to poorer orbital coverage there (Agapitov et al., 2011). It could easily lead to a factor of 2 underestimation of wave ampli-

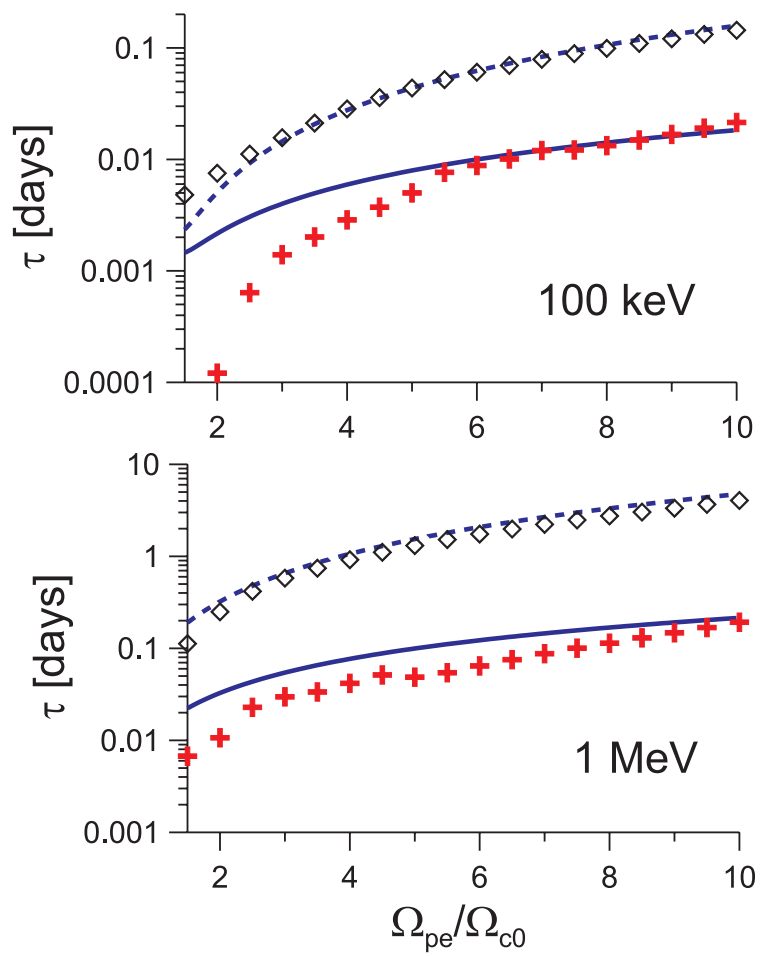

Fig. 14. Electron lifetimes for two values of particle energy, for quasi-parallel and oblique chorus waves. Full numerical solutions as well as the corresponding analytical estimates in Eqs. (9) and (12) are displayed as a function of $\Omega_{\mathrm{pe}}$.

tudes there, so that actual lifetimes may be expected to remain within a factor of 2 of the ones obtained for a constant $B_{\mathrm{w}}$ even at low energy $E \sim 100 \mathrm{keV}$ (moreover, nightside chorus is generally slightly more intense near the equator in Cluster data than the dayside chorus considered here, which dominates at higher latitudes; see Artemyev et al., 2012b). Since analytical lifetimes are slightly larger than full numerical values for constant $B_{\mathrm{w}}(\lambda)$ and low to medium energy electrons, they should represent good estimates of the actual lifetimes.

\subsection{Comparison with a numerical parameterization of lifetimes in the outer belt}

The analytical estimate in Eq. (9) of electron lifetimes for small $\theta$-waves is compared with parameterized lifetimes recently obtained by fitting numerical calculations for an interaction with lower-band chorus waves dominant in the outer radiation belt. We use the same initial conditions as Shprits et al. (2007) and Gu et al. (2012): 100 pT storm-time chorus waves, dayside plasma trough density $N_{\mathrm{e}} \sim 100(3 / L)^{4} \mathrm{~cm}^{-3}$ for $L>3$ from Sheeley et al. (2001), and $\theta<\pi / 4$. Moreover, chorus waves are assumed here to be uniformly present up to $\lambda^{+} \approx 40^{\circ}$, while in reality intensity peaks between $\lambda \approx 15^{\circ}$ and $\lambda \approx 30^{\circ}$ on the dayside (Gu et al., 2012). While upper-band chorus is also assumed to be present in the 

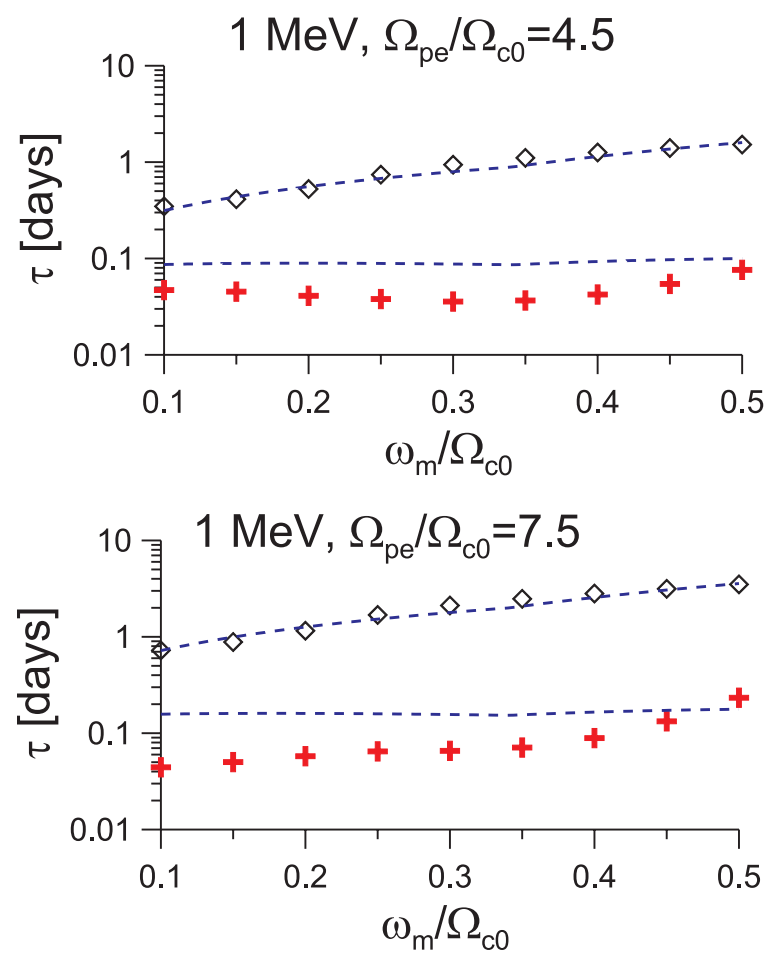

Fig. 15. Electron lifetimes for two values of ratio $\Omega_{\mathrm{pe}} / \Omega_{\mathrm{c} 0}$, for quasi-parallel (diamonds) and oblique (red crosses) chorus waves. Full numerical solutions as well as the corresponding analytical estimates in Eqs. (9) and (12) are displayed as a function of mean frequency $\omega_{\mathrm{m}}$.

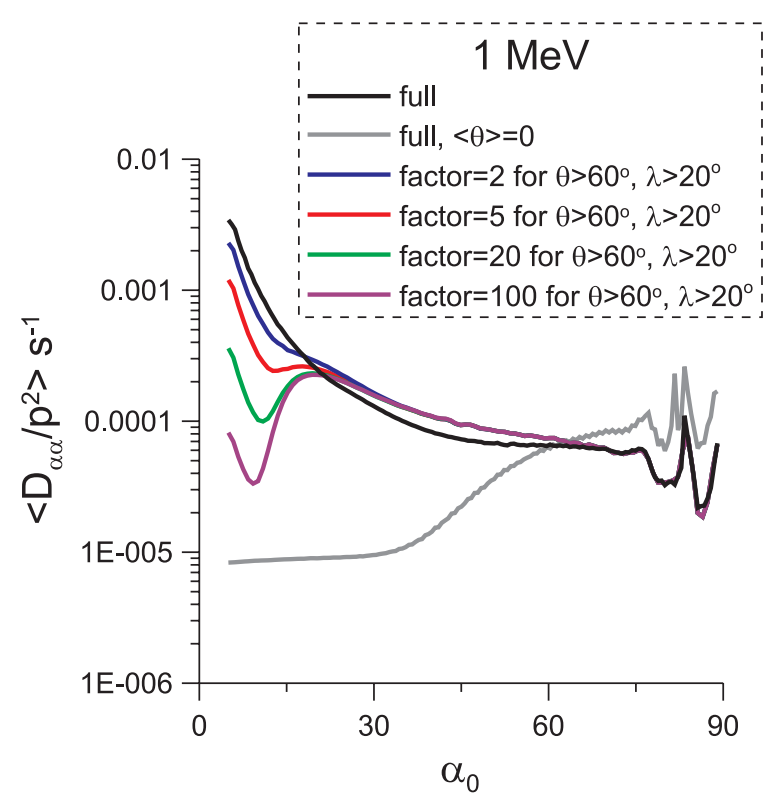

Fig. 16. Comparison of bounce-averaged pitch-angle diffusion coefficients for $\Omega_{\mathrm{pe}} / \Omega_{\mathrm{c} 0}=4.5$ and $1 \mathrm{MeV}$ electrons, obtained for different reduced levels of oblique chorus wave intensity at $\lambda>20^{\circ}$.
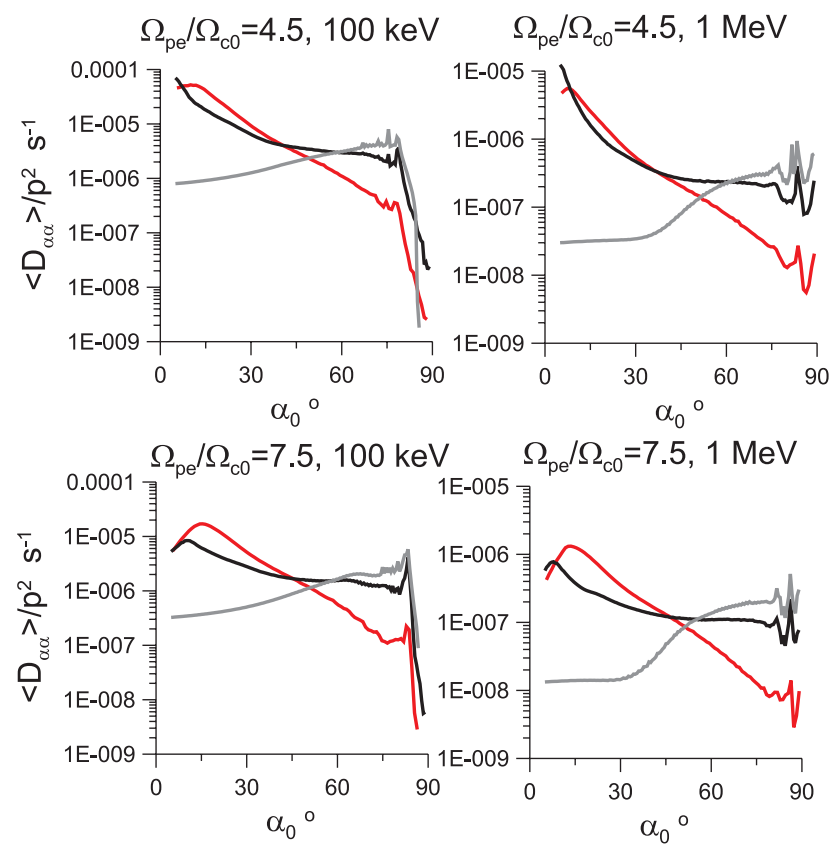

Fig. 17. Diffusion coefficients calculated with realistic root-meansquare wave amplitudes $B_{\mathrm{W}}(\lambda)$ for $L=4$ to 5 for $\mathrm{Kp}<3$ are shown by red curves. Black and grey curves show $\langle D\rangle_{\mathrm{B}}$ calculated with a constant rms $B_{\mathrm{W}}=6 \mathrm{pT}$ with a realistic $g(\theta)$ and with $\langle\theta\rangle \sim 0$, respectively.

numerical simulations by Gu et al. (2012), its effect on lifetimes consists mainly in the suppression of the deep gap in diffusion between the Landau and first cyclotron resonance peaks. In such a case, Landau resonance is unimportant in lifetimes for moderately oblique waves at $E>100 \mathrm{keV}$, as noted by Gu et al. (2012). Upper-band chorus actually plays the same role in the outer belt as lightning-generated waves in the plasmasphere (which fill the trough in diffusion between Landau and cyclotron peaks of hiss waves; see Meredith et al., 2007). For analytical estimates, only the cyclotron part of the lifetime in Eq. (9) is therefore considered here.

The numerically parameterized lifetime (Gu et al., 2012) (of $50 \%$ accuracy) is fairly recovered by the analytical estimate in Eq. (9)-(14) in Fig. 18, in spite of our very rough approximation of initial conditions. Moreover, the variations with density and $L$ shell given by Gu et al. (2012) have also been checked to be similar (or identical as concerns $B_{0}$ dependency) to the variations of our analytical lifetimes. Note that part of the discrepancy may also originate in the assumption made by Gu et al. (2012) that lifetimes can be calculated as $\tau \approx 1 /\langle D\rangle\left(\alpha_{\mathrm{LC}}\right)$. While the latter formula is generally accurate in the considered cases, it can lead to some differences with Eq. (7), which is used in our analytical and numerical calculations and is thought to be more consistently accurate (Albert and Shprits, 2009). 


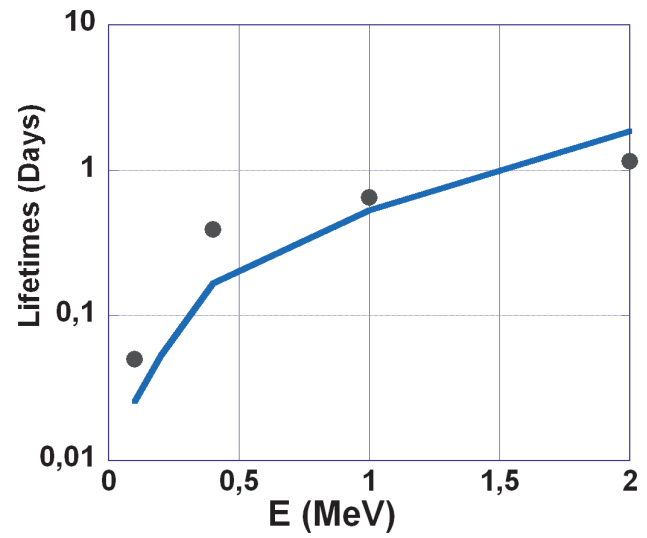

Fig. 18. Comparison of analytical lifetime with parameterized lifetimes obtained by fitting numerical simulations for $\Omega_{\mathrm{pe}} / \Omega_{\mathrm{c} 0}=4.5$ at $L=4.5$ as a function of energy on the dayside. The analytical estimate in Eq. (9) is plotted (blue line for $100 \mathrm{pT}$ average intensity lower-band chorus with $\omega_{\mathrm{m}} / \Omega_{\mathrm{c} 0} \sim 0.3$ ) as well as the parameterized lifetime (solid black circles) for moderately oblique waves.

\subsection{Interaction with plasmaspheric hiss and lightning- generated waves}

Meredith et al. (2007) have shown that the $G_{\omega}$ distribution of whistler waves in the slot region $(L \in[2,3])$ can be approximated by a sum of three Gaussians with different mean values and variances. Here we compare diffusion coefficients calculated with this approximation and with an approximation of $G_{\omega}$ by a sum of two Gaussians (see description in Sect. 4.2). We consider a $g(\theta)$ distribution obtained from Cluster data (see Eq. 18) and the simplified approximation from Meredith et al. (2007) with $\langle\theta\rangle=0$ and $X_{\mathrm{w}}=0.36$. All calculations in Fig. 19 are performed for $L=2.0$. We can conclude that approximating $G_{\omega}$ by a sum of two Gaussians gives almost the same diffusion coefficients as for three Gaussians (Meredith et al., 2007). Moreover, including the oblique waves observed by Cluster does not change $\langle D\rangle_{\mathrm{B}}$ substantially (compare left and right columns in Fig. 19). Therefore, one can safely use a quasi-parallel approximation of wave propagation for the calculation of analytical lifetimes. The effect of oblique waves is less important here than in the case of chorus at $L \sim 4$ (see Sect. 5), because the portion of wave power inside the large- $\theta$ range (above the Gendrin angle) is much smaller for lower-frequency hiss and lightning-generated waves.

Analytical and full numerical lifetimes in the slot region at $L=2$ and 2.5 under quiet geomagnetic conditions are displayed in Fig. 20 as a function of energy (with plasma/wave parameters given in Sect. 4.2; see Meredith et al., 2007). CRRES spectra were fitted by two Gaussians corresponding respectively to hiss and lightning-generated waves (see Sect. 4). Here we use $g(\theta)$ determined by Eq. (18) from Cluster statistics. However, comparisons with numerical lifetimes obtained by Meredith et al. $(2007,2009)$ show also a
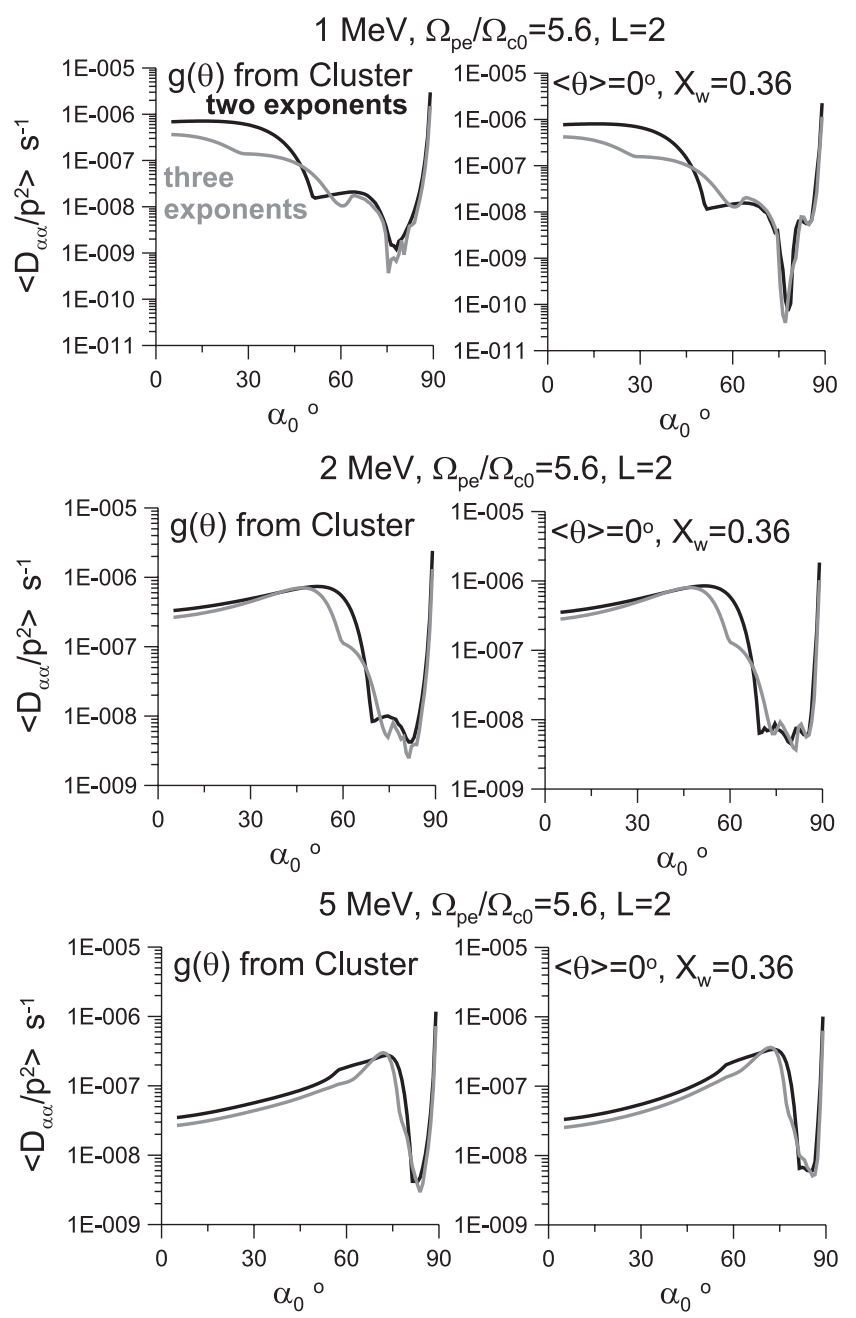

Fig. 19. $\langle D\rangle_{\mathrm{B}}$ for three values of electron energy $(L=2.0$, $\left.\Omega_{\mathrm{pe}} / \Omega_{\mathrm{c} 0}=5.6\right)$. Approximation of $G_{\omega}$ by three and two exponents are used (see text for details). Left column show data obtained with approximation of $g(\theta)$ distribution in Eq. (18). Right column shows data for parallel wave propagation.

good agreement. Analytical lifetimes reproduce rather precisely the full numerical solutions at medium to high energy where both cyclotron and Landau resonance are present. At lower energy where the Landau resonance contributes alone, actual lifetimes are less accurately estimated by the analytical formulas (as explained in Appendix B). But this occurs only for very long lifetimes (which are therefore practically useless).

To sum up the results of our numerous comparisons with numerical simulations, Table 2 provides a brief overview of the parameter ranges where analytical lifetime estimates can be considered as good approximations of the full numerical calculations in the plasmasphere as well as in the outer belt. 


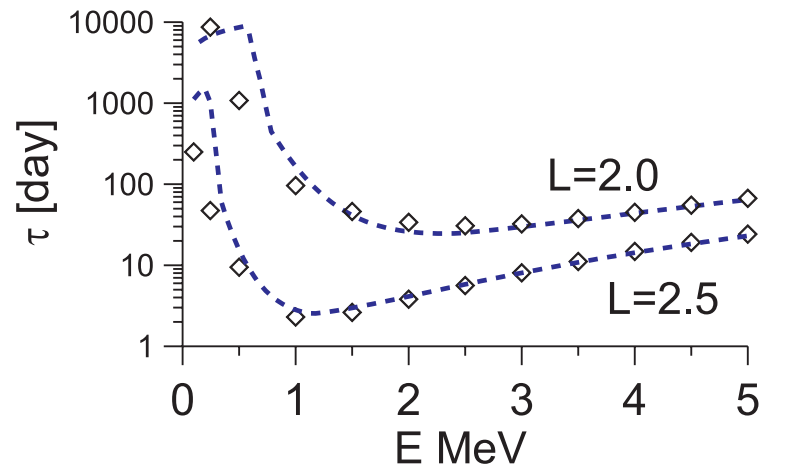

Fig. 20. Analytical lifetimes at $L=2$ and 2.5 given by Eq. (9) (dashed blue curve) are compared with those obtained by the numerical scheme: black diamonds show results obtained with a realistic $\theta$ distribution and $|n| \leq 25\left(N_{\mathrm{r}}<10\right)$. Electron energy varies between $100 \mathrm{keV}$ and $5 \mathrm{MeV}$. Hiss and lightning-generated whistlers are considered.

\subsection{Global analytical lifetimes compared to recent mea- surements at $E \sim 300 \mathrm{keV}$ and $2 \mathrm{MeV}$}

It is interesting to compare the analytical lifetime estimates discussed above with recent statistics of lifetimes obtained from SAC-C and DEMETER satellites for $L<5$ (Benck et al., 2010) and from the SCATHA/SC3 satellite at $L>5$ (Su et al., 2012) for $300 \mathrm{keV}$ electrons, and with SAMPEX lifetimes for $E \sim 2 \mathrm{MeV}$ (Meredith et al., 2009; Tu et al., 2010). To this aim, the root-mean-square amplitudes of lower-band chorus measured by Cluster in the dayside outer belt $(L>4)$ during moderate geomagnetic activity periods ( $\mathrm{Kp} \sim 1.5$ to 2$)$ have been fitted by a formula $B_{\mathrm{w}} \sim$ $20 \mathrm{pT} \cdot \exp (-|L-7| / 2)$, showing a maximum of wave intensity at $L \sim 7$ and yielding the same values as in Fig. 2 for $L \sim 4.5$. For $L>4$, the outer-belt density model $N_{\mathrm{e}} \sim$ $100(3 / L)^{4} \mathrm{~cm}^{-3}$ from Sheeley et al. (2001) is used. To better model a smooth transition between oblique chorus and more quasi-parallel chorus from $L=5$ to 6 , Eq. (15) is used to estimate lifetimes for $L=4$ to 5, while for $L>5$ we use a rough approximation $\tau \sim \tau_{s+l}+\left(\tau_{s} / 2\right) \min (L-5,1)$ from Eqs. (14)-(15). This way, $\tau \sim \tau_{\mathrm{s}} / 2 \gg \tau_{s+l}$ for $L>5.5$. Inside the plasmasphere $(L<3.5)$, we consider the quiet-time hiss and lightning-generated whistler spectra (and plasma densities) supplied by Meredith et al. (2007) from CRRES observations and lifetime estimates in Eqs. (9) and (14) for weakly oblique waves.

The quiet-time lifetimes measured in the outer belt are reasonably well recovered by the analytical estimates in Fig. 21 . Analytical estimates of the lifetimes of $300 \mathrm{keV}$ electrons remain within a factor of 2 of the actual lifetimes for $L=4$ to 8 and roughly reproduce their overall decline toward higher $L$ shells (Su et al., 2012; Benck et al., 2010). For $2 \mathrm{MeV}$ electrons, we only took lifetimes estimated from optimum model fitting to SAMPEX data during two pre-storm periods shown
Table 2. Parameter domains where analytical lifetime estimates are a good approximation for the full numerical simulations.

\begin{tabular}{lc}
\hline Spatial region & Validity range of analytical estimates \\
\hline $2 \leq L<3$ (plasmasphere) & $0.25<E(\mathrm{MeV}) \leq 5$ \\
\multicolumn{2}{c}{ with only hiss and lightning-generated waves $(100 \mathrm{~Hz}$ to $6 \mathrm{kHz})$} \\
$4 \leq L<7$ (outer belt) & $\omega_{\mathrm{m}} / \Omega_{\mathrm{c} 0}=0.1$ to 0.5 \\
& $\Omega_{\mathrm{pe}} / \Omega_{\mathrm{c} 0}=2$ to 10 \\
Oblique chorus & $\max \left(0.1, \Omega_{\mathrm{c} 0}^{3} /\left(\Omega_{\mathrm{pe}}^{2} \omega_{\mathrm{m}}\right)\right)<E(\mathrm{MeV})<5$ \\
Quasi-parallel chorus & $0.1 \leq E(\mathrm{MeV}) \leq 5$ \\
\hline
\end{tabular}

in Figs. 5 and 7 in the work by Tu et al. (2010), corresponding to low geomagnetic activity. Although experimental lifetimes may then vary by a factor of 5 (Borovsky and Denton, 2009), analytical lifetime estimates remain within a factor of 2 of the average values inferred from model fitting to measurements in these two cases. The increase of analytical lifetimes with energy in the region $4<L<6.6$ is also in qualitative agreement with observations (Su et al., 2012; Meredith et al., 2007).

For $L=2$ to 3, Fig. 22 shows that analytical lifetime estimates derived for hiss and lightning-generated waves such that $\Delta \theta=30^{\circ}$ and $\theta_{\text {Max }}^{s}=45^{\circ}$ (Meredith et al., 2007) are again in reasonable agreement with the measured lifetimes for $E=2 \mathrm{MeV}$, although the fitting of the actual spectra that is used (only two Gaussians; see Sect. 4.2) may be too rough and the (quiet-time) wave intensities used slightly too small. It is also clear from Eqs. (9) and (11) that small lifetimes at $L<2.5$ obtain only for small $\Delta \theta<45^{\circ}$ at $\alpha_{0}>70^{\circ}$, in order for the minimum of diffusion between the cyclotron peak and the Landau peak to be not too deep. The presence of a multiplicative factor $\tan \Delta \theta$ at the numerator of Eq. (11) in analytical lifetimes, which comes from Eq. (8), actually explains the larger lifetimes obtained by Meredith et al. (2009) in numerical simulations when considering high-frequency waves with $\Delta \theta>45^{\circ}$. Moreover, cyclotron resonance for $\alpha_{0}>70^{\circ}$ occurs only at $\lambda<10^{\circ}$ (from adiabatic invariance). The small lifetimes measured at $L=2$ to 3 therefore require high-frequency whistlers such that $\Delta \theta<30^{\circ}$ at $\lambda<10^{\circ}$. It is precisely what was observed onboard CRRES and Cluster (see Fig. 3). In fact, lightning-induced whistlers do not need to be guided at $\lambda>10^{\circ}$ to yield small lifetimes; they must only be quasi-parallel near the equator. For high energies $E>1 \mathrm{MeV}$, the Landau term $\tau_{\text {Landau }}$ in Eq. (9) is actually negligible at $L>2$, and hiss and lightning-generated waves seem to be sufficient to explain quiet-time electron losses in the slot region (Meredith et al., 2007, 2009; Kim et al., 2011). At lower energies $E<1 \mathrm{MeV}$, it is not true anymore if one considers only hiss and lightning-generated waves such that $f<6 \mathrm{kHz}$ as in CRRES spectra from Meredith et al. (2007). Nevertheless, VLF waves from ground transmitters are also important at $L<3$ (Abel and Thorne, 1998; Starks et al., 2008; Breneman et al., 2011; Cohen et al., 2012), as well as magnetosonic waves at $L>2$ (Meredith et al., 2009). 


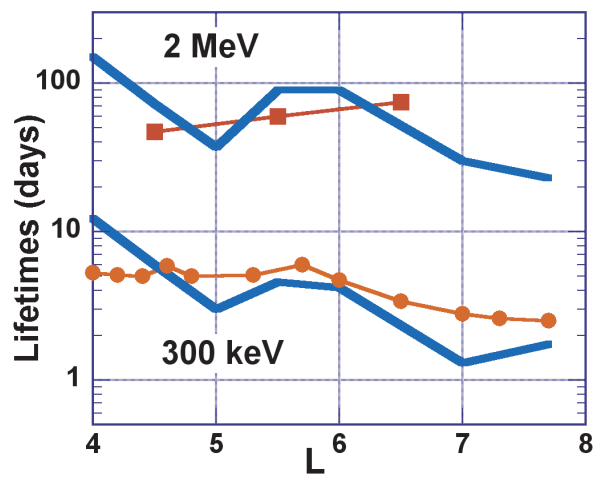

Fig. 21. Analytical lifetime estimates from $L=4$ to 8 (blue curves) are compared with actual lifetimes measured by SAC-C, DEMETER, and SCATHA (Benck et al., 2010; Su et al., 2012) for $300 \mathrm{keV}$ electrons and with lifetimes obtained during two pre-storm periods by model fitting to SAMPEX data (Tu et al., 2010) for $2 \mathrm{MeV}$ electrons. Lifetimes are estimated for lower-band chorus wave average amplitudes obtained from Cluster for moderate geomagnetic activity $(\mathrm{Kp} \sim 2)$.

Both these kinds of waves play essentially the same role as lightning-generated whistler waves in filling the gap in diffusion rate between the Landau and cyclotron peaks corresponding to hiss waves, but at higher pitch angles (near $\alpha_{0}=90^{\circ}$ ) or in the same range of pitch angles at smaller energy. Although the exact level of these waves is not well known (highly variable), we simply assume here that it is sufficient to get $\tau_{\text {Landau }} \ll \tau_{\mathrm{s}}$ in Eq. (9). This corresponds to additional wave amplitudes around $3-5 \mathrm{pT}$ typically. In Fig. 22, analytical lifetime estimates for $E=300 \mathrm{keV}$ are therefore plotted assuming that this term $\tau_{\text {Landau }}$ is negligible in Eq. (9). With this assumption, the increase of measured lifetimes as energy decreases for $L<2.6$ can be understood from analytical estimates. The decrease with $E$ of the maximum equatorial pitch angle $\left(\cos \alpha_{\mathrm{UC}, 1} \propto 1 / p\right)$ where cyclotron resonance can occur for the low-frequency part of the spectrum (hiss waves) first leads to an increase of the important $\Delta \tau_{i}$ term (from lightning-generated waves) in Eqs. (11) and (9), because $\left(1-\sin \alpha_{U C, 1}\right)$ increases faster than $1 / p$. At still smaller energies $(300 \mathrm{keV})$, cyclotron resonance is not available anymore for hiss waves: the cyclotron term in Eq. (9) from hiss waves is replaced by the same term from higher-frequency and much smaller intensity lightninggenerated waves, leading to an even greater increase of lifetimes.

\section{Conclusions}

In this paper, analytical estimates of energetic electron lifetimes in the radiation belts have been compared extensively with full numerical simulations. We consider low-frequency $\left(\Omega_{\mathrm{ci}} \ll \omega<\Omega_{\mathrm{c}} / 2, \omega \Omega_{\mathrm{c}} \ll \Omega_{\mathrm{pe}}^{2}\right)$ nearly parallel as well as oblique whistler waves, such as those observed in the inner

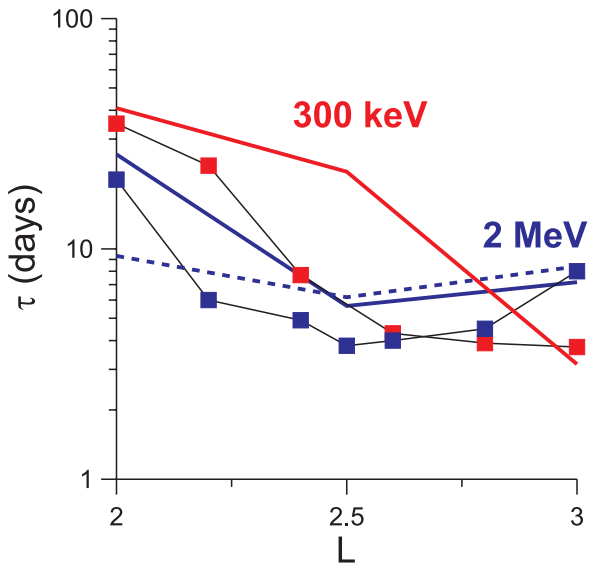

Fig. 22. Analytical lifetime estimates from Eq. (9) for $L=2$ to 3 are compared with actual lifetimes measured by SAC-C and DEMETER (Benck et al., 2010) for $300 \mathrm{keV}$ electrons and by SAMPEX (Meredith et al., 2007) for $2 \mathrm{MeV}$ electrons. Lifetimes are estimated for interaction with quiet-time hiss and lightning-generated whistlers, with an additional contribution from higher-frequency whistler waves or magnetosonic waves required at low energy (see text). Blue color is for $2 \mathrm{MeV}$ and red color for $300 \mathrm{keV}$, with squares indicating measured values. The dashed line shows the lifetimes from Eq. (14) alone.

belt by CRRES (Meredith et al., 2007) and in the outer belt by Cluster and THEMIS (Agapitov et al., 2012b; Li et al., 2011). The considered wave-normal distribution, frequency spectra, and wave power latitudinal distribution have been obtained from statistics of various satellite measurements. The analytical model relies mainly on the possibility of representing the spectra as a sum of relatively narrow Gaussians such that $\Delta \omega \sim \omega_{\mathrm{m}} / 2$, on the approximation of Bessel functions by their series expansions around their first maxima, and on the use of a simplified dispersion relation (which should remain valid for wave-normal angles not too close to the resonance cone angle at very high latitudes). The refined analytical model described here captures many features of the full numerical solution in a wide parameter range. The main results of the present study are summarized below:

1. Inside the plasmasphere, the analytical model reproduces accurately numerical lifetime variations over a few decades as a function of energy $(0.1<E(\mathrm{MeV}) \leq$ 5) and $L \sim 2$ to 3 (corresponding to $\Omega_{\mathrm{pe}} / \Omega_{\mathrm{c} 0} \sim 5$ to 8 ), when considering a realistic spectrum composed of hiss $\left(\omega / \Omega_{\mathrm{c} 0} \sim 0.005\right.$ to 0.02$)$ and lightning-generated $\left(\omega / \Omega_{\mathrm{c} 0} \sim 0.01\right.$ to 0.1$)$ whistler-mode waves of realistic latitude-varying obliqueness (obtained from Cluster and CRRES statistics during relatively quiet periods).

2. In the outer belt at $L \sim 4$ to 5.5 , the analytical model recovers rather accurately lifetime variations over a few decades as a function of energy ( $E=0.1$ to $5 \mathrm{MeV}$ ), density $\left(\Omega_{\mathrm{pe}} / \Omega_{\mathrm{c} 0} \sim 2\right.$ to 10$)$, and mean frequency 
$\omega / \Omega_{\mathrm{c} 0}=0.1$ to 0.5 , when considering realistic oblique chorus waves obtained from Cluster statistics during moderately disturbed periods.

3. When considering quasi-parallel chorus waves corresponding to quiet-time Cluster statistics at $L \sim 5.5$ to 7 , the analytical lifetime estimates are very accurate in general in the same ranges of energy, density, and frequency as above.

4. Numerical as well as analytical calculations show that timescales are strongly reduced at $L=4$ to 5.5 when chorus waves reach large wave-normal angles, between the Gendrin and resonance angles. Such very oblique whistler waves are often present at moderate to high latitudes, where they represent from $10 \%$ to $50 \%$ of reported chorus occurrences (Burton and Holzer, 1974; Hayakawa et al., 1990; Santolík et al., 2009; Agapitov et al., 2012b; Haque et al., 2010, 2011). The damping of oblique chorus waves by suprathermals is much more efficient on the nightside and at $L>5.5$ than at $L<5.5$ on the dayside (Chen et al., 2013), which probably explains why their intensity remains similar to the intensity of quasi-parallel waves in the latter case for $\mathrm{Kp}<3$ in the Cluster statistics presented here.

5. At $L=4$ to 7 , lifetime estimates obtained for average chorus intensities and wave-normal distributions are in reasonable agreement with lifetimes measured during relatively quiet periods, for both $300 \mathrm{keV}$ and $2 \mathrm{MeV}$ electrons.

6. With very oblique chorus waves, the strong diffusion regime can be reached easily in the dayside outer belt at times of high geomagnetic activity. This could correspond to some of the very rapid drop-outs in trapped electron density at $L=4$ to 5.5 observed by various satellites during disturbed periods.

7. Inside the plasmasphere $(2<L<3)$, the small lifetimes measured by satellites require high-frequency whistler waves (lightning-induced and VLF) that have to remain very moderately oblique at latitudes within $10^{\circ}$ of the equator, as actually observed by Cluster. Measured lifetimes are in good agreement with analytical estimates for $2 \mathrm{MeV}$ electrons when considering only hiss and lightning-generated waves. For $300 \mathrm{keV}$ electrons at $L \leq 2.5$, higher-frequency VLF waves must be added to hiss and lightning-generated waves to recover the observed lifetimes.

Space weather forecasting and nowcasting capabilities are very much in demand today to help safeguard important satellite assets from strong particle flux hazards linked to solar activity. In this context, the proposed analytical estimates may prove useful for quickly evaluating the sensitivity of global radiation belt codes results to insufficiently known wave and plasma parameters, for thorough investigations of different processes, as well as more simply to speed up the calculations. Although analytical estimates cannot be expected to be as accurate as full numerical calculations, analytical lifetimes have been demonstrated here to remain within a factor of 2 of the exact solutions over a very wide parameter range. Such a discrepancy is much smaller than the uncertainties associated with wave and plasma models. Since the analytical lifetime model provides estimates as a function of all the different wave and plasma parameters, it can be used with any plasma density profile and with any average wave intensity distribution (as a function of MLT, $L$, and geomagnetic conditions). Parameterized wave amplitudes inferred from CRRES observations as a function of geomagnetic activity (Kp) have already been supplied by Shprits et al. (2007) in their Eq. (6) for dayside chorus. Depending on the actual MLT repartition of chorus waves, the resulting lifetimes should be multiplied in addition by a factor of 2 to 4 . Inside the plasmasphere, typical wave spectra and intensities can be found in the work by Meredith et al. (2007) for $L=2$ to 3 .

The proposed estimates could also help to fully take advantage of future space weather satellite measurements, allowing immediate evaluation of diffusion coefficients and lifetimes directly inside global radiation belt forecasting codes as a function of time-varying (with geomagnetic activity) wave spectra and wave-normal distributions as soon as they become available. Nevertheless, magnetosonic and EMIC waves may also play an important role in the dynamics of the radiation belts (Summers et al., 2007; Li et al., 2007; Meredith et al., 2009). It would be interesting to derive similar analytical models for these waves in a future work.

\section{Appendix A}

\section{On the evaluation of lifetimes from diffusion coefficients}

Albert and Shprits (2009) have derived a simplified and very useful formula for calculating the lifetime of electrons via the simple integration of the inverse of the bounce-averaged pitch-angle diffusion coefficient multiplied by $\tan \alpha_{0}$. However, for typical cases of interest, their expression in Eq. (11) is only valid to within a factor of 2 , since their own fully numerical lifetimes are generally roughly 2 times smaller when the minimum of $\langle D\rangle_{\mathrm{B}} \tan \alpha_{0}$ occurs at small to moderate pitch angles. Hence the multiplying factor $\sigma \sim 1 / 2$ is used in Mourenas et al. (2012b) as well as here in Eqs. (7)-(12). It is worth noting that, for oblique lower-band chorus-electron interaction, a simplified form of the bounce-averaged cyclotron diffusion coefficient can be derived, such that $\langle D\rangle_{\mathrm{B}} \sim$ $\langle D(\pi / 4)\rangle_{\mathrm{B}}\left(\sin (\pi / 4) / \sin \left(\alpha_{0}\right)\right)^{P}$, with $P=1$ to 2 from analytical calculations in Mourenas et al. (2012b,a). In this case, it is actually possible to estimate rather accurately the lifetime as the inverse of the lowest eigenvalue of the diffusion 
operator (see details in Schulz, 1991). With the new variable $y=\sin \alpha_{0}$, one gets $\left\langle D_{z z}\right\rangle_{\mathrm{B}}=\left(1-y^{2}\right)(y T(y))^{2}\langle D\rangle_{B} \propto$ $\left(1-y^{2}\right)=z$ (compare with Eqs. (190) to (197) from Schulz, 1991). One then finds the following (analytically for $P=2$ as well as numerically for $P=1$ ) from Eqs. (194) and (197) in the work by Schulz (1991):

$\tau \sim\left(\int_{\sin \alpha_{\operatorname{Min}}}^{1} \frac{4 d y /(3 \pi)}{\sqrt{\left(1-y^{2}\right)\langle D\rangle_{\mathrm{B}}}}\right)^{2} \sim \frac{32 \cos ^{2} \alpha_{\mathrm{Min}}}{9 \pi^{2}\langle D\rangle_{B}(\pi / 4)}$,

where $\cos \alpha_{\text {Min }} \sim 0.96$ for $L>3.5$ for a lower bound of integration $\alpha_{\mathrm{Min}} \leq \pi / 12$ (Mourenas et al., 2012b) and $3 \pi / 4$ is the first zero of the Bessel function $J_{0}$. From Eqs. (3), (15), and (16) from Mourenas et al. (2012b), one obtains a new estimate of the multiplying factor $\sigma \approx 64 \cos ^{2}(\pi / 12) /\left(9 \pi^{2}\right) \approx$ $2 / 3$. Nevertheless, exact numerical calculation of the lifetimes yields generally slightly smaller lifetimes than the inverse of the lowest eigenvalue, as can be seen in Fig. 1 in the work by Albert and Shprits (2009) for a model diffusion coefficient corresponding precisely to such an eigenfunction decomposition. In fact, one may expect the actual lifetime to be slightly smaller because of the effect of the other, higher eigenvalues. The numerical lifetime plotted in Fig. 1 in the work by Albert and Shprits (2009) is actually 0.8 times smaller than the theoretical one, yielding finally $\sigma \approx 5 / 9$, which is very close to our assumed value $\sigma \approx 1 / 2$ for the cyclotron terms related to oblique chorus waves. Turning now to nearly parallel hiss waves near the loss cone at $L<3.5$, one gets now $D \propto D\left(\alpha_{\mathrm{LC}}\right) / \cos ^{2} \alpha_{0}$ from Eq. (33) in the work by Mourenas and Ripoll (2012), leading to a multiplying factor $\sigma \approx 0.8\left(8 / \pi^{2}\right) \approx 1 / 2$ again for the cyclotron part.

\section{Appendix B}

Rough evaluation of high-latitude Landau resonance effects at low $L$

It is worth noting that the analytical model of Landau resonance lifetime provided in Eq. (9) with $F_{\mathrm{hl}}=1$ (equivalent to Eq. 36 in the work by Mourenas and Ripoll, 2012) is correct only when Landau resonance occurs not too far from the equator. Since the resonant value of pitch angle $\alpha_{\mathrm{R}}$ from the combination of Eq. (3) and simplified dispersion relation with $n=0$ is given by $\tan \alpha_{\mathrm{R}} \approx \sin \alpha_{0} \Omega_{\mathrm{c} 0} p \varepsilon_{\mathrm{m} 0} /\left(\gamma \omega_{\mathrm{m}}\right)$ (see Mourenas et al., 2012b), resonance occurs off the equator $\left(\sin \alpha_{\mathrm{R}}>\sin \alpha_{0}\right)$ when $\cos \alpha_{0}>\gamma \omega_{\mathrm{m}} /\left(\Omega_{\mathrm{c} 0} p \varepsilon_{\mathrm{m} 0}\right)$. Furthermore, taking into account the inequalities $p<\gamma$ and $\omega_{\mathrm{m}} \ll$ $\Omega_{\mathrm{c} 0}$ for hiss, lightning-generated waves, and lower-band chorus, together with the resonance condition, implies that $\tan \alpha_{\mathrm{R}} \gg \sin \alpha_{0}$ and therefore $\sin \alpha_{\mathrm{R}} \sim 1$ in general. Now, $D(n=0, \lambda)$ varies roughly like $\left(\Omega_{\mathrm{c}} / \Omega_{\mathrm{c} 0}\right)^{1 / 2}$ for $p \varepsilon_{\mathrm{m} 0}>$ $1.84 / \tan \Delta \theta$ and like $\left(\Omega_{\mathrm{c} 0} / \Omega_{\mathrm{c}}\right)^{4}$ for $p \varepsilon_{\mathrm{m} 0}<1.84 / \tan \Delta \theta$ (where one must take also $\Delta \theta \leq 45^{\circ}$ for these formulas to be valid; see Mourenas and Ripoll, 2012). Consequently, a significant effect from off-equatorial resonance can occur only for small values of $p \varepsilon_{\mathrm{m} 0}<1.84$ (i.e., small energy, density and frequency). The magnitude of $\langle D\rangle(n=0)$ will be noticeably modified when $\Omega_{\mathrm{c}} / \Omega_{\mathrm{c} 0} \geq 1.5$ to 2 . Using adiabatic invariance with $\sin \alpha_{\mathrm{R}} \sim 1$, this last condition is equivalent to $\alpha_{0}<45-55^{\circ}$. However, if cyclotron resonance is effective enough (with a significant diffusion rate such that $D(n<0)>D(n=0))$ for $\alpha_{0}>45^{\circ}-55^{\circ}$, there will be no consequence on the lifetime estimate. This means that lifetime estimates in Eqs. (9)-(12) remain roughly correct as long as $\max \left(p \varepsilon_{\mathrm{m} 0, i}\right)>2^{1 / 4}$, in order for cyclotron resonance to be important enough at moderate pitch angles. In the opposite limit, Eq. (10) for the Landau part with $F_{\mathrm{hl}}=1$ leads to very long lifetimes (typically $>100$ days inside the plasmasphere), which can nevertheless be sometimes strongly underestimated. Equations (34)-(36) in the work by Mourenas and Ripoll (2012) have indeed been derived under the assumption that the Landau resonance part of $D$ makes a significant contribution in lifetimes only at not too high latitudes. When this assumption is violated at moderate $\alpha_{0}$ and low $L$ inside the plasmasphere, one must integrate in the lifetime Eq. (7) a term $1 /\left(\langle D\rangle_{\mathrm{B}} \tan \alpha_{0}\right) \propto \cos ^{4} \alpha_{0} / \sin ^{8} \alpha_{0}$ (instead of $\cos ^{4} \alpha_{0}$ ) coming from the last term of Eq. (25) in the work by Mourenas and Ripoll (2012) (where $g_{\mathrm{s}} \sim 0$ and $\left.\Omega_{\mathrm{c}}\left(\lambda_{\min }\right) / \Omega_{\mathrm{c} 0} \approx 1 / \sin ^{2} \alpha_{0}\right)$. This leads to a lifetime multiplicative factor $\approx 1 /\left(10 \sin ^{7} \alpha_{0}^{\mathrm{Max}}\right)$ varying between 10 and 5000 for a lower bound of integration $\alpha_{0}^{\mathrm{Max}}=\alpha_{\mathrm{LC}} \sim 9^{\circ}$ to $30^{\circ}$ at $L \sim 1.5$ to 3.5 . But radiation belt simulations are rarely run over more than one-year duration (Subbotin et al., 2011). Thus, using an approximate multiplicative factor $F_{\mathrm{hl}} \approx \max \left(10 \Theta\left(\pi / 4-\alpha_{0}^{\mathrm{Max}}\right), 1\right)$ (with $\Theta$ the Heaviside function, such that $\Theta(x)=0$ for $x<0$ and $\Theta=1$ otherwise) should be sufficient in general for calculations over less than one year. Moreover, higher-frequency waves (such as lightning-generated whistlers or VLF; see Meredith et al., 2009) should most often prevent lifetimes from becoming too large, in agreement with recent satellite measurements of electron lifetimes (see Sect. 5.7).

Acknowledgements. The authors would like to thank the reviewers for valuable suggestions to improve the manuscript. The work of K.V.V. was partially supported by The Ministry of Education and Science of Russian Federation, project 8527.

Topical Editor L. Blomberg thanks two anonymous referees for their help in evaluating this paper.

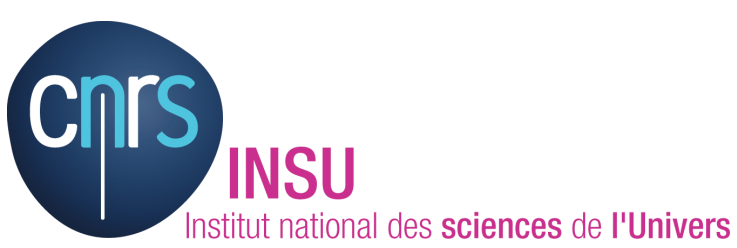

The publication of this article is financed by CNRS-INSU. 


\section{References}

Abel, B. and Thorne, R. M.: Electron scattering loss in Earth's inner magnetosphere 1. Dominant physical processes, J. Geophys. Res., 103, 2385-2396, doi:10.1029/97JA02919, 1998.

Agapitov, O., Krasnoselskikh, V., Khotyaintsev, Y. V., and Rolland, G.: A statistical study of the propagation characteristics of whistler waves observed by Cluster, Geophys. Res. Lett., 382, L20103, doi:10.1029/2011GL049597, 2011.

Agapitov, O., Artemyev, A., Krasnoselskikh, V., Khotyaintsev, Y. V.,Mourenas, D., Breuillard, H., Balikhin, M., and Rolland, G.: Statistics of Whistler-Mode Waves in the Outer Radiation Belt: Cluster STAFF-SA measurements, J. Geophys. Res., submitted, 2013a.

Agapitov, O., Krasnoselskikh, V., Khotyaintsev, Y. V., and Rolland, G.: Correction to "A statistical study of the propagation characteristics of whistler waves observed by Cluster", Geophys. Res. Lett., 39, L24102, doi:10.1029/2012GL054320, 2012 b.

Albert, J. M.: Evaluation of quasi-linear diffusion coefficients for whistler mode waves in a plasma with arbitrary density ratio, J. Geophys. Res., 110, A03218, doi:10.1029/2004JA010844, 2005.

Albert, J. M.: Simple approximations of quasi-linear diffusion coefficients, J. Geophys. Res., 112, A12202, doi:10.1029/2007JA012551, 2007.

Albert, J. M.: Dependence of quasi-linear diffusion coefficients on wave parameters, J. Geophys. Res., 117, A09224, doi:10.1029/2012JA017718, 2012.

Albert, J. M. and Shprits, Y. Y.: Estimates of lifetimes against pitch angle diffusion, Journal of Atmospheric and Solar-Terrestrial Physics, 71, 1647-1652, doi:10.1016/j.jastp.2008.07.004, 2009.

Artemyev, A., Agapitov, O., Breuillard, H., Krasnoselskikh, V., and Rolland, G.: Electron pitch-angle diffusion in radiation belts: The effects of whistler wave oblique propagation, Geophys. Res. Lett., 39, L08105, doi:10.1029/2012GL051393, 2012a.

Artemyev, A., Agapitov, O., Krasnoselskikh, V., Breuillard, H., and Rolland, G.: Statistical model of electron pitch-angle diffusion in the outer radiation belt, J. Geophys. Res., 117, A08219, doi:10.1029/2012JA017826, 2012b.

Barker, A. B., Li, X., and Selesnick, R. S.: Modeling the radiation belt electrons with radial diffusion driven by the solar wind, Space Weather, 3, S10003, doi:10.1029/2004SW000118, 2005.

Benck, S., Mazzino, L., Cyamukungu, M., Cabrera, J., and Pierrard, V.: Low altitude energetic electron lifetimes after enhanced magnetic activity as deduced from SAC-C and DEMETER data, Ann. Geophys., 28, 849-859, doi:10.5194/angeo-28-849-2010, 2010.

Borovsky, J. E. and Denton, M. H.: Electron loss rates from the outer radiation belt caused by the filling of the outer plasmasphere: The calm before the storm, J. Geophys. Res., 114, A11203, doi:10.1029/2009JA014063, 2009.

Bortnik, J., Inan, U. S., and Bell, T. F.: Landau damping and resultant unidirectional propagation of chorus waves, Geophys. Res. Lett., 33, L03102, doi:10.1029/2005GL024553, 2006.

Bortnik, J., Chen, L., Li, W., Thorne, R. M., and Horne, R. B.: Modeling the evolution of chorus waves into plasmaspheric hiss, J. Geophys. Res., 116, A08221, doi:10.1029/2011JA016499, 2011a.

Bortnik, J., Chen, L., Li, W., Thorne, R. M., Meredith, N. P., and Horne, R. B.: Modeling the wave power distribution and characteristics of plasmaspheric hiss, J. Geophys. Res., 116, A12209, doi:10.1029/2011JA016862, $2011 \mathrm{~b}$.
Boskova, J., Jiricek, F., Triska, P., Lundin, B. V., and Shkliar, D. R.: A possible common nature of equatorial half-gyrofrequency VLF emissions and discrete plasmaspheric emissions, Ann. Geophys., 8, 755-763, 1990.

Breneman, A., Cattell, C., Wygant, J., Kersten, K., Wilson, III, L. B., Schreiner, S., Kellogg, P. J., and Goetz, K.: Large-amplitude transmitter-associated and lightning-associated whistler waves in the Earth's inner plasmasphere at $L>2$, J. Geophys. Res., 116, A06310, doi:10.1029/2010JA016288, 2011.

Breuillard, H., Zaliznyak, Y., Krasnoselskikh, V., Agapitov, O., Artemyev, A., and Rolland, G.: Chorus wave-normal statistics in the Earth's radiation belts from ray tracing technique, Ann. Geophys., 30, 1223-1233, doi:10.5194/angeo-30-1223-2012, 2012.

Bunch, N. L., Spasojevic, M., and Shprits, Y. Y.: Off-Equatorial Chorus Occurrence and Wave Amplitude Distributions as Observed by the Polar Plasma Wave Instrument, J. Geophys. Res., 117, A04205, doi:10.1029/2011JA017228, 2012.

Burton, R. K. and Holzer, R. E.: The Origin and Propagation of Chorus in the Outer Magnetosphere, J. Geophys. Res., 79, 10141023, doi:10.1029/JA079i007p01014, 1974.

Chen, L., Thorne, R. M., Li, W., and Bortnik, J.: Modeling the Wave Normal Distribution of Chorus Waves, J. Geophys. Res., in press, doi:10.1002/jgra.50086, 2013.

Choi, H.-S., Lee, J., Cho, K.-S., Kwak, Y.-S., Cho, I.-H., Park, Y.D., Kim, Y.-H., Baker, D. N., Reeves, G. D., and Lee, D.-K.: Analysis of GEO spacecraft anomalies: Space weather relationships, Space Weather, 9, S06001, doi:10.1029/2010SW000597, 2011.

Chum, J. and Santolík, O.: Propagation of whistler-mode chorus to low altitudes: divergent ray trajectories and ground accessibility, Ann. Geophys., 23, 3727-3738, doi:10.5194/angeo-23-37272005, 2005.

Cohen, M. B., Lehtinen, N. G., and Inan, U. S.: Models of Ionospheric VLF Absorption of Powerful Ground Based Transmitters, Geophys. Res. Lett., 39, L24101, doi:10.1029/2012GL054437, 2012.

Cornilleau-Wehrlin, N., Chanteur, G., Perraut, S., Rezeau, L., Robert, P., Roux, A., de Villedary, C., Canu, P., Maksimovic, M., de Conchy, Y., Hubert, D., Lacombe, C., Lefeuvre, F., Parrot, M., Pinçon, J. L., Décréau, P. M. E., Harvey, C. C., Louarn, Ph., Santolik, O., Alleyne, H. St. C., Roth, M., Chust, T., Le Contel, O., and STAFF team: First results obtained by the Cluster STAFF experiment, Ann. Geophys., 21, 437-456, doi:10.5194/angeo-21437-2003, 2003.

Davidson, G. T.: An improved empirical description of the bounce motion of trapped particles, J. Geophys. Res., 81, 4029, doi:10.1029/JA081i022p04029, 1976.

Denton, R. E., Takahashi, K., Galkin, I. A., Nsumei, P. A., Huang, X., Reinisch, B. W., Anderson, R. R., Sleeper, M. K., and Hughes, W. J.: Distribution of density along magnetospheric field lines, J. Geophys. Res., 111, A04213, doi:10.1029/2005JA011414, 2006.

Fok, M.-C., Glocer, A., Zheng, Q., Horne, R. B., Meredith, N. P., Albert, J. M., and Nagai, T.: Recent developments in the radiation belt environment model, J. Atmos. Solar-Terr. Phys., 73, 14351443, doi:10.1016/j.jastp.2010.09.033, 2011.

Gendrin, R.: Le guidage des whistlers par le champ magnetique, Planet. Space Sci., 5, 274, doi:10.1016/0032-0633(61)90096-4, 1961. 
Ginzburg, V. L. and Rukhadze, A. A.: Waves in magnetoactive plasma, Nauka, Moscow, 2nd revised edition edn., 1975.

Gkioulidou, M., Wang, C.-P., Wing, S., Lyons, L., Wolf, R., and Hsu, T.-S.: Effect of an MLT dependent electron loss rate on the magnetosphere-ionosphere coupling, J. Geophys. Res., 117, A11218, doi:10.1029/2012JA018032, 2012.

Glauert, S. A. and Horne, R. B.: Calculation of pitch angle and energy diffusion coefficients with the PADIE code, J. Geophys. Res., 110, A04206, doi:10.1029/2004JA010851, 2005.

Gu, X., Shprits, Y. Y., and Ni, B.: Parameterized lifetime of radiation belt electrons interacting with lower-band and upperband oblique chorus waves, Geophys. Res. Lett., 39, L15102, doi:10.1029/2012GL052519, 2012.

Haque, N., Spasojevic, M., Santolík, O., and Inan, U. S.: Wave normal angles of magnetospheric chorus emissions observed on the Polar spacecraft, J. Geophys. Res., 115, A00F07, doi:10.1029/2009JA014717, 2010.

Haque, N., Inan, U. S., Bell, T. F., Pickett, J. S., Trotignon, J. G., and Facskó, G.: Cluster observations of whistler mode ducts and banded chorus, Geophys. Res. Lett., 38, L18107, doi:10.1029/2011GL049112, 2011.

Hattori, K., Hayakawa, M., Lagoutte, D., Parrot, M., and Lefeuvre, F.: Further evidence of triggering chorus emissions from wavelets in the hiss band, Planet. Space Sci., 39, 1465-1472, doi:10.1016/0032-0633(91)90075-L, 1991.

Hayakawa, M., Hattori, K., Shimakura, S., Parrot, M., and Lefeuvre, F.: Direction finding of chorus emissions in the outer magnetosphere and their generation and propagation, Planet. Space Sci., 38, 135-137, doi:10.1016/0032-0633(90)90012-F, 1990.

Helliwell, R. A.: Whistlers and Related Ionospheric Phenomena, Stanford University Press, USA, 1965.

Horne, R. B., Thorne, R. M., Glauert, S. A., Albert, J. M., Meredith, N. P., and Anderson, R. R.: Timescale for radiation belt electron acceleration by whistler mode chorus waves, J. Geophys. Res., 110, A03225, doi:10.1029/2004JA010811, 2005.

Iucci, N., Levitin, A. E., Belov, A. V., Eroshenko, E. A., Ptitsyna, N. G., Villoresi, G., Chizhenkov, G. V., Dorman, L. I., Gromova, L. I., Parisi, M., Tyasto, M. I., and Yanke, V. G.: Space weather conditions and spacecraft anomalies in different orbits, Space Weather, 3, S01001, doi:10.1029/2003SW000056, 2005.

Kennel, C. F. and Petschek, H. E.: Limit on Stably Trapped Particle Fluxes, J. Geophys. Res., 71, 1-28, 1966.

Kim, K.-C., Shprits, Y., Subbotin, D., and Ni, B.: Understanding the dynamic evolution of the relativistic electron slot region including radial and pitch angle diffusion, J. Geophys. Res., 116, A10214, doi:10.1029/2011JA016684, 2011.

Kondrashov, D., Ghil, M., and Shprits, Y.: Lognormal Kalman filter for assimilating phase space density data in the radiation belts, Space Weather, 9, S11006, doi:10.1029/2011SW000726, 2011.

Lauben, D. S., Inan, U. S., Bell, T. F., and Gurnett, D. A.: Source characteristics of ELF/VLF chorus, J. Geophys. Res., 107, 1429, doi:10.1029/2000JA003019, 2002.

Li, W., Shprits, Y. Y., and Thorne, R. M.: Dynamic evolution of energetic outer zone electrons due to wave-particle interactions during storms, J. Geophys. Res., 112, A10220, doi:10.1029/2007JA012368, 2007.

Li, W., Bortnik, J., Thorne, R. M., and Angelopoulos, V.: Global distribution of wave amplitudes and wave normal angles of chorus waves using THEMIS wave observations, J. Geophys. Res.,
116, A12205, doi:10.1029/2011JA017035, 2011.

Lyons, L. R.: Pitch angle and energy diffusion coefficients from resonant interactions with ion-cyclotron and whistler waves, J. Plasma Phys., 12, 417-432, doi:10.1017/S002237780002537X, 1974.

Lyons, L. R., Thorne, R. M., and Kennel, C. F.: Electron pitch-angle diffusion driven by oblique whistler-mode turbulence., J. Plasma Phys., 6, 589-606, doi:10.1017/S0022377800006310, 1971.

Lyons, L. R., Thorne, R. M., and Kennel, C. F.: Pitch-angle diffusion of radiation belt electrons within the plasmasphere., J. Geophys. Res., 77, 3455-3474, doi:10.1029/JA077i019p03455, 1972.

Ma, Q., Ni, B., Tao, X., and Thorne, R. M.: Evolution of the plasma sheet electron pitch angle distribution by whistler-mode chorus waves in non-dipole magnetic fields, Ann. Geophys., 30, 751760, doi:10.5194/angeo-30-751-2012, 2012.

Meredith, N. P., Horne, R. B., and Anderson, R. R.: Substorm dependence of chorus amplitudes: Implications for the acceleration of electrons to relativistic energies, J. Geophys. Res., 106, 13165-13178, doi:10.1029/2000JA900156, 2001.

Meredith, N. P., Horne, R. B., Glauert, S. A., and Anderson, R. R.: Slot region electron loss timescales due to plasmaspheric hiss and lightning-generated whistlers, J. Geophys. Res. (Space Physics), 112, A08214, doi:10.1029/2007JA012413, 2007.

Meredith, N. P., Horne, R. B., Glauert, S. A., Baker, D. N., Kanekal, S. G., and Albert, J. M.: Relativistic electron loss timescales in the slot region, J. Geophys. Res., 114, A03222, doi:10.1029/2008JA013889, 2009.

Mourenas, D. and Ripoll, J.-F.: Analytical estimates of quasilinear diffusion coefficients and electron lifetimes in the inner radiation belt, J. Geophys. Res., 117, A01204, doi:10.1029/2011JA016985, 2012.

Mourenas, D., Artemyev, A., Agapitov, O., and Krasnoselskikh, V.: Acceleration of radiation belts electrons by oblique chorus waves, J. Geophys. Res., 117, A10212, doi:10.1029/2012JA018041, 2012a.

Mourenas, D., Artemyev, A. V., Ripoll, J.-F., Agapitov, O. V., and Krasnoselskikh, V. V.: Timescales for electron quasi-linear diffusion by parallel and oblique lower-band Chorus waves., J. Geophys. Res., 117, A06234, doi:10.1029/2012JA017717, $2012 \mathrm{~b}$.

Nemec, F., Santolik, O., Parrot, M., Pickett, J. S., Hayosh, M., and Cornilleau-Wehrlin, N.: Conjugate observations of quasiperiodic emissions by Cluster and DEMETER spacecraft, J. Geophys. Res., 118, 198-208, doi:10.1029/2012JA018380, 2012.

Ni, B., Thorne, R. M., Shprits, Y. Y., and Bortnik, J.: Resonant scattering of plasma sheet electrons by whistler-mode chorus: Contribution to diffuse auroral precipitation, Geophys. Res. Lett., 35, L11106, doi:10.1029/2008GL034032, 2008.

Ni, B., Thorne, R. M., Meredith, N. P., Shprits, Y. Y., and Horne, R. B.: Diffuse auroral scattering by whistler mode chorus waves: Dependence on wave normal angle distribution, J. Geophys Res., 116, A10207, doi:10.1029/2011JA016517, 2011.

Ni, B., Thorne, R. M., and Ma, Q.: Bounce-averaged Fokker-Planck diffusion equation in non-dipolar magnetic fields with applications to the Dungey magnetosphere, Ann. Geophys., 30, 733750, doi:10.5194/angeo-30-733-2012, 2012.

Orlova, K. G. and Shprits, Y. Y.: Dependence of pitch-angle scattering rates and loss timescales on the magnetic field model, Geophys. Res. Lett., 37, L05105, doi:10.1029/2009GL041639, 2010. 
Orlova, K. G., Shprits, Y. Y., and Ni, B.: Bounce-averaged diffusion coefficients due to resonant interaction of the outer radiation belt electrons with oblique chorus waves computed in a realistic magnetic field model, J. Geophys. Res., 117, A07209, doi:10.1029/2012JA017591, 2012.

Ozhogin, P., Tu, J., Song, P., and Reinisch, B. W.: Field-aligned distribution of the plasmaspheric electron density: An empirical model derived from the IMAGE RPI measurements, J. Geophys. Res., 117, A06225, doi:10.1029/2011JA017330, 2012.

Reeves, G. D., Chen, Y., Cunningham, G. S., Friedel, R. W. H., Henderson, M. G., Jordanova, V. K., Koller, J., Morley, S. K., Thomsen, M. F., and Zaharia, S.: Dynamic Radiation Environment Assimilation Model: DREAM, Space Weather, 10, S03006, doi:10.1029/2011SW000729, 2012.

Santolík, O., Gurnett, D. A., Pickett, J. S., Chum, J., and CornilleauWehrlin, N.: Oblique propagation of whistler mode waves in the chorus source region, J. Geophys. Res., 114, A00F03, doi:10.1029/2009JA014586, 2009.

Sazhin, S. S. and Horne, R. B.: Quasilongitudinal approximation for whistler-mode waves in the magnetospheric plasma, Planet. Space Sci., 38, 1551-1553, doi:10.1016/0032-0633(90)90160-R, 1990.

Schulz, M.: The magnetosphere., in: Geomagnetism, edited by: Jacobs, J. A., pp. 87-293, 1991.

Sheeley, B. W., Moldwin, M. B., Rassoul, H. K., and Anderson, R. R.: An empirical plasmasphere and trough density model: CRRES observations, J. Geophys. Res., 106, 25631-25642, doi:10.1029/2000JA000286, 2001.

Shklyar, D. and Matsumoto, H.: Oblique Whistler-Mode Waves in the Inhomogeneous Magnetospheric Plasma: Resonant Interactions with Energetic Charged Particles, Surv. Geophys., 30, 55104, doi:10.1007/s10712-009-9061-7, 2009.

Shklyar, D. R., Chum, J., and Jiřriček, F.: Characteristic properties of $\mathrm{Nu}$ whistlers as inferred from observations and numerical modelling, Ann. Geophys., 22, 3589-3606, doi:10.5194/angeo-223589-2004, 2004.

Shprits, Y. Y. and Ni, B.: Dependence of the quasi-linear scattering rates on the wave normal distribution of chorus waves, J. Geophys. Res., 114, A11205, doi:10.1029/2009JA014223, 2009.

Shprits, Y. Y., Thorne, R. M., Horne, R. B., and Summers, D.: Bounce-averaged diffusion coefficients for fieldaligned chorus waves, J. Geophys. Res., 111, A10225, doi:10.1029/2006JA011725, 2006.

Shprits, Y. Y., Meredith, N. P., and Thorne, R. M.: Parameterization of radiation belt electron loss timescales due to interactions with chorus waves, Geophys. Res. Lett., 34, L11110, doi:10.1029/2006GL029050, 2007.

Shprits, Y. Y., Menietti, J., Gu, X., Kim, K.-C., and Horne, R.: Gyro-resonant interactions between the radiation belt electrons and whistler mode chorus waves in the radiation environments of Earth, Jupiter, and Saturn, a comparative study, J. Geophys. Res., 117, A11216, doi:10.1029/2012JA018031, 2012.

Starks, M. J., Quinn, R. A., Ginet, G. P., Albert, J. M., Sales, G. S., Reinisch, B. W., and Song, P.: Illumination of the plasmasphere by terrestrial very low frequency transmitters: Model validation, J. Geophys. Res., 113, A09320, doi:10.1029/2008JA013112, 2008.
Su, Y.-J., Johnston, W. R., Albert, J. M., Ginet, G. P., Starks, M. J., and Roth, C. J.: SCATHA measurements of electron decay times at $5<L<8$, J. Geophys. Res., 117, 8212, doi:10.1029/2012JA017685, 2012.

Subbotin, D. A., Shprits, Y. Y., and Ni, B.: Long-term radiation belt simulation with the VERB 3-D code: Comparison with CRRES observations, J. Geophys. Res., 116, A12210, doi:10.1029/2011JA017019, 2011.

Summers, D.: Quasi-linear diffusion coefficients for field-aligned electromagnetic waves with applications to the magnetosphere, J. Geophys. Res., 110, A08213, doi:10.1029/2005JA011159, 2005.

Summers, D. and Ni, B.: Effects of latitudinal distributions of particle density and wave power on cyclotron resonant diffusion rates of radiation belt electrons, Earth, Planets, Space, 60, 763-771, 2008.

Summers, D., Ni, B., and Meredith, N. P.: Timescales for radiation belt electron acceleration and loss due to resonant wave-particle interactions: 2. Evaluation for VLF chorus, ELF hiss, and electromagnetic ion cyclotron waves, J. Geophys. Res., 112, A04207, doi:10.1029/2006JA011993, 2007.

Tao, X., Bortnik, J., Albert, J. M., Liu, K., and Thorne, R. M.: Comparison of quasilinear diffusion coefficients for parallel propagating whistler mode waves with test particle simulations, Geophys Res. Lett., 38, L06105, doi:10.1029/2011GL046787, 2011.

Thorne, R. M.: Radiation belt dynamics: The importance of wave-particle interactions, Geophys. Res. Lett., 372, L22107, doi:10.1029/2010GL044990, 2010.

Thorne, R. M. and Horne, R. B.: Landau damping of magnetospherically reflected whistlers, J. Geophys. Res., 99, 17249, doi:10.1029/94JA01006, 1994.

Trakhtengerts, V. Y.: Stationary states of the Earth's outer radiation zone, Geomagnetism and Aeronomy, 6, 827-836, 1966.

Tsurutani, B. T. and Smith, E. J.: Two types of magnetospheric ELF chorus and their substorm dependences, J. Geophys. Res., 82, 5112-5128, doi:10.1029/JA082i032p05112, 1977.

Tsurutani, B. T., Verkhoglyadova, O. P., Lakhina, G. S., and Yagitani, S.: Properties of dayside outer zone chorus during HILDCAA events: Loss of energetic electrons, J. Geophys. Res., 114, A03207, doi:10.1029/2008JA013353, 2009.

Tu, W., Li, X., Chen, Y., Reeves, G. D., and Temerin, M.: Stormdependent radiation belt electron dynamics, J. Geophys. Res., 114, A02217, doi:10.1029/2008JA013480, 2009.

Tu, W., Selesnick, R., Li, X., and Looper, M.: Quantification of the precipitation loss of radiation belt electrons observed by SAMPEX, J. Geophys. Res., 115, A07210, doi:10.1029/2009JA014949, 2010.

Varotsou, A., Boscher, D., Bourdarie, S., Horne, R. B., Meredith, N. P., Glauert, S. A., and Friedel, R. H.: Three-dimensional test simulations of the outer radiation belt electron dynamics including electron-chorus resonant interactions, J. Geophys. Res., 113, A12212, doi:10.1029/2007JA012862, 2008.

Xiao, F., Su, Z., Zheng, H., and Wang, S.: Modeling of outer radiation belt electrons by multidimensional diffusion process, J. Geophys. Res., 114, A03201, doi:10.1029/2008JA013580, 2009. 